\title{
Zur juraksamojedischen Konjugation
}

Vor mehr als einem Jahrhundert sind - herausgegeben von Anton Schiefner, dem Mitglied der Petersburger Akademie der Wissenschaften, - die "Grammatik dersamojedischen Sprachen" (St. Petersburg 1854) und die "Wörterverzeichnisse aus den samojedischen Sprachen» (ebd. 1855) von M. Alexander Castrén erschienen. Castrén, der am 7. Mai 1852 im Alter von 38 Jahren starb, hatte vom Juli 1842 bis zum Frühjahr 1844 in Nordrussland und Sibirien, später vom März 1845 bis Anfang 1849 in Sibirien unter ausserordentlich ungünstigen Verhältnissen und zum Teil von einer schweren Krankheit geplagt an Ort und Stelle Studien getrieben. Von seiner insgesamt anderthalb Jahrzehnte währenden wissenschaftlichen Tätigkeit widmete er fast zehn Jahre dem Studium der samojedischen Sprachen.

Obwohl im Arbeitsplan des aus Finnland stammenden Akademiemitglieds Sjögren für Castrén das Sammeln von Texten nicht erwähnt wird, sammelte der geniale und über ein beachtliches wissenschaftliches Rüstzeug verfügende junge Forscher auch ein überaus wertvolles Material an Volksdichtung: er zeichnete juraksamojedische Heldenlieder, epische und andere Lieder und auch vier selkupische Heldenlieder und mehrere kleinere Lieder auf. Dieses Material gilt heute als Sprachdenkmal. Die von Castrén aufgezeichneten Lieder wurden von Toivo Lehtisalo herausgegeben. ${ }^{1}$ Lehtisalo trieb in tundra- und waldjurakischen Sprachgebieten, in den nördlichsten Gegenden des Europäischen Russland und in Sibirien, in den Jahren 19111912 Sammelarbeit im Terrain. Sein wertvolles Material an Volksdichtung erschien in feiner Transkription in einem dicken

\footnotetext{
${ }^{1}$ Samojedische Volksdichtung. Gesammelt von M. A. Castrén. Herausgegeben von T. Leнtisalo. Helsinki 1940. MSFOu LXXXIII; XXV, 355 S. - Abgekürzt C und Seitenzahl.
} 
Band im Jahre 1947, sein umfangreiches Wörterbuch aber i.J. 1956. ${ }^{1}$ Ausser den obigen Werken von Castrén und Lehtisalo ist auch der von Lehtisalo herausgegebene Band "Samojedische Sprachmaterialien" ein unentbehrliches Quellenwerk ${ }^{2}$, das sämtliche selkupischen und waldjurakischen lexikalischen und grammatischen Materialien Castréns enthält, ausserdem zwei Varianten eines selkupischen lyrischen Gesanges, aufgezeichnet von Castrén, ferner Lehtisalos jurakische Texte, die er i.J. 1912 aufgezeichnet, aber bis dahin nicht herausgegeben hatte. In diesem Band sind auch Lehtisalos selkupische Aufzeichnungen aus dem Jahre 1957 zu finden.

Nach einem mehrere Jahre umfassenden Studium der Texte von Castrén und Lehtisalo erkannte ich, dass die Texte unsere Kenntnisse über die jurakische Konjugation wesentlich vermehren und zugleich auch modifizieren. Ohne Zweifel hat Castrén viel mehr von den Eigentümlichkeiten der samojedischen Verben gesehen, als die Grammatik enthält. Er hat auch vieles in der Erinnerung bewahrt, hatte aber weder Kraft noch Zeit es niederzuschreiben, geschweige denn seine Texte grammatisch zu verwerten. - In dieser Studie teile ich meine Beobachtungen und meine aus den sprachlichen Fakten ziehbaren Schlussfolgerungen mit. Ich will das Mitzuteilende einfach und deutlich ausdrücken, und meide deshalb absichtlich den Gebrauch der modernen Fachwörter; ist doch selbst ein so allgemein gebrauchtes Fachwort wie "Aspekt" in der finnischugrischen Sprachwissenschaft nicht eindeutig. Ich bin überzeugt, dass der grundsätzliche Standpunkt Castréns noch nicht veraltet ist, den er so formuliert hat: "Je ne suis pas ennemi de la philosophie, à laquelle j'ai au contraire consacré la majeure partie de mes années d'étude; mais je préfère voir la philosophie d'un côté, la philologie de l'autre... J'aime, en un mot, à exprimer une pensée claire en termes clairs, et ne puis souffrir la manière de certains, qui par la foule des termes techniques et des expressions philosophiques, rendent mystérieuses

1 Juraksamojedische Volksdichtung MSFOu XC; XII, $615 \mathrm{~S}$. Abgekürzt: Seitenzahl. Juraksamojedisches Wörterbuch (Lexica Societatis Fenno-Ugricae XIII) CIX, 601 S. Abgekürzt: Wb

2 MSFOu 122 (1960) 463 S. Abgekürzt: Mat 
les notions courantes de la grammaire." (Zitiert aus der französischen Ausgabe der Gedächtnisrede von E. N. Setälä: JSFOu XXX,1 b S. 38).

\section{Das juraksamojedische Nomen-Verbum}

Es ist eine allgemein anerkannte Ansicht, dass die Kategorien des Nomens und des Verbs in den uralischen Sprachen ursprünglich zusammenfielen. Das Fachwort Nomen-Verbum hat $\mathrm{K}$. B. Wiklund zuerst in seinem Artikel "Nomen-verba im Lappischen" (JSFOu X, 210-216) gebraucht. In jurakischer Hinsicht ist Wiklunds Bemerkung zu den Wortpaaren jellu'bersten; Borst, Spalte' und kiellu- 'gerinnen (von Blut); geronnenes Blut' sehr aufschlussreich: "in den Wörtern jellu-, kielluist vielleicht das Nomen als ein aus dem Verbum deriviertes Nomen actionis auf $-\imath$ anzusehen» (S. 213). Unter Wiklunds Beispielen gibt es Wortpaare, in denen die beiden Glieder nicht identisch sind: vein $-u$ des Verbalstammes entspricht einem - $a,-e$, oder selten -es des Nominalstammes; hier ist vielleicht das $-u$ eher als ein besonderes verbbildendes Suffix anzusehen" (S. 216).

Im Jurakischen kann ich Nomen-Verba von dem Typ wie ung. fagy 'Frost; erfrieren', les 'Lauer; auf etwas lauern', die keinerlei Suffix erhalten, nicht nachweisen. Ein solches ist wahrscheinlich selkupisch (Mat 38) N MO ķods, Tsch ķooče, OO kৃooč 'Schneegestöber': N kodse-k, kodśa-n 'ist Schneegestöber' (in den beiden letzteren Wörtern kann $a, e$ auch zur Endung gehören). Doch sind beim Wortpaar NP kooču, koču-ng, kooču-ng sowohl das Nomen als auch das Verb Verbalnomina auf ${ }^{*} \beta$. Dem selkupischen Nomen entspricht im Tundrajurakischen (Wb 175) $\chi \bar{a} \delta_{\perp}^{p}, \chi \bar{a} \delta$, im Waldjurakischen $k \bar{a} t t^{p}, k \bar{a} t$, vgl. noch OP $\chi \bar{a} \delta \delta p^{\hat{\jmath}}$ (Wb 63), Arch $\chi \bar{a} \delta a-\dot{m} i^{\prime}$ 'mein Schneegestöber' (182). Vgl. kam. (D-J) kadan 'frischgefallener Schnee (im Herbst)'. In den verbalen Formen erscheint ein mit dem Ableitungssuffix $* \beta$ gebildeter Verbalnomenstamm, an den sich das verbalnomenbildende Suffix $m a(+j)$ anschliesst:

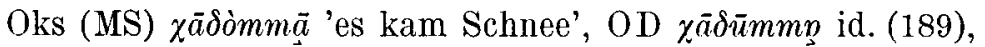
W Nj kìtummã- $\beta$ ' 'ist Schnee gekommen?' 
Die juraksamojedischen Nomen-Verba erfordern mehr Erklärung als diejenigen der finnisch-ugrischen Sprachen. ${ }^{1}$

'Schweiss; schwitzen' (Wb 329). Das jurakische Wort (Sprogis) Kan neho 'Schweiss' hat im Kamassischen eine Entsprechung:(C) nogo, (D-J) nuжo, vgl. Koib. nogo, Mot. niugu (Beitr. $59)$. Sowohl die Nomina als die Verbalstämme sind Verbalnomina auf * $\beta$ : jur. 0 ńó $\chi^{-}-$, no $\chi^{-} \bar{o}^{-},($Mat 239) njoho-, vgl. kam.

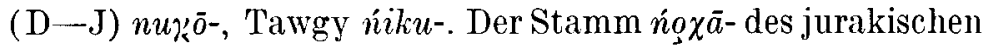
Wortes MS no $\chi \bar{a}-m$ ' 'ich geriet . . in Schweiss' (297) ist aber ein Verbalnomen auf $* j \varepsilon(i)$; es bedeutet auch soviel wie 'mein Schwitzen'. Den Vokalismus der ersten Silbe kann man auf Grund des Diphthongs iu des Nomens Mot. niugu verstehen: die erste Silbe dürfte im Jurakischen und im Tawgy *nŭu ${ }^{*} n \check{o}$ - gewesen sein. Das kurze $\check{\imath}$ Element des Diphthongs kam in enge Verbindung mit dem anlautenden $* n$ - und führte nach Verschmelzung damit den Lautwandel ${ }^{*} n$ - $>n$ - herbei. Im

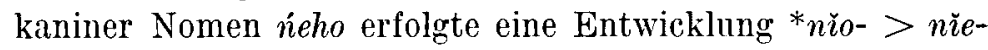
$>>n e-$, im Tawgy aber ${ }^{*} n \grave{\imath} e->>n i$ - Ein sekundäres $n^{-}$ enthält z.B. auch das jur. Wort ne 'Weib', in Castréns Texten nie, im Kaniner Heldenlied nì̀ (215), doch auch $n \dot{n}$ - $\delta ә з$ (214).

'Feuer; brennen' ( fi. tule-). Das jurakische Wort $t \bar{u}$, tù (Wb 495) hat auch eine verbale Form: O tī- 'brennen', T' $t \bar{u} \beta \beta a i_{\smile} t \bar{u}$ 'das Feuer [eig. unser Feuer] brennt', Kan (Sjo) tū-t’śe_barànnā, lējnāa 'es brennt mit grosser Flamme, lodert' [eig. brennend brennt es, flammt], WS tùr' $u u^{\prime}$ 'jon! 'zünde das Feuer an!' Vgl. (C) tu-ton ( tu-tun) tu-mah 'das Feuer begann zu brennen' [war brennend] (123). Die bekannten samojedischen Entsprechungen des Wortes sind: jen. $t \bar{u}, t u$, Tawgy tui. Ich halte es für wahrscheinlich, dass dem ursam. Nomen-Verbum *tul(e) ein verbalnomenbildendes Suffix *je $(i)$ angehängt wurde: ${ }^{*} t u l-j \varepsilon>>t u l>t u i$ (anders CompGr.

1 Bei der begrenzten Vereinfachung der Transkription Lehtisalos folge ich mit einer unwesentlichen Abweichung dem Verfahren von Bo Wickman (FUF XXXIII). - Abkürzung der Dialekte: $\mathrm{O}=$ Dialekt von Obdorsk; $\mathrm{OD}=$ Ob-Delta; $\mathrm{T}=$ Tas; $\mathrm{PD}=$ Pur-Delta; $\mathrm{MS}=$ Malaja Semlja; BS = Bolschaja Semlja; U = Ustje; Kan = Kanin; Arch $=$ Archangelsk; $\mathrm{W}=$ waldjurakisch; $\mathrm{Ni}=$ nittśe-Fl.; $\mathrm{Nj}=\mathrm{Njalina} ; \mathrm{Ij}$. $=$ Ljamin-Fl. (vgl. Lehtisalo: Juraksam. Volksdichtung VII-X). 
$\$ 199$ ). Der Diphthong wurde im Tawgy und im WS im Verbalstamm erhalten, während er sich in den anderen samojedischen Sprachen mit dem Vokal der ersten Silbe verband und so dessen Längung herbeiführte.

'Fisch; fischen' (Wb 165-166). Das uralische Wort ist ein ursprünglicher a-Stamm: fi. kala, kam. (C) kola, (D-J) kiōsa, $l^{n} n \overline{0}: l a^{a}$ (Koib. Klapr. kola). Im Kamassischen existiert das Wort auch als ein mit dem Suffix *je (i) gebildeter Verbalstamm: ko.lei-lam 'ich fische'. Bei dieser Angabe kann man schon beobachten, dass aus der Verbindung des Auslautvokals $a$ mit dem Suffix ${ }^{*} j$. ein Diphthong $-e i$ - entstanden ist. Nach einer lexikalischen Angabe Lehtisalos wird das Wort auch im Jurakischen als ein Verb gebraucht: 'T $\chi \bar{a} l l \bar{e}$ 'er fischt', und dies ist auch ein Verbalnomen auf ${ }^{*} j \varepsilon$. Das Wort 'Fisch' erscheint bei Budenz in mehreren Varianten, eine von diesen ist hale, und die verbale Form dazu hali-. (In Lehtisalos selkupischen Aufzeichnungen ist das Nomen-Verbum léesi 'Fisch' $\sim$ kiẹli-'fischen' im wesentlichen identisch mit dem Wortpaar, das Budenz aufgezeichnet hat.) Alle diese nominalen und verbalen Formen entsprechen dem kamassischen Verbalstamm liolei-. Es lässt sich auch mit anderen Angaben belegen, dass das Wort 'Fisch' im Jurakischen ein ursprünglicher $a$ -

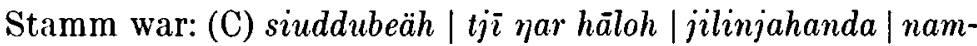
setida 'Der Riese so viel Fische | lebendig isst' (174). Die Form hāloh lässt sich auf die Form *hāloj < *hālaj zurückführen. Die Form (Wb) U $\chi \bar{a} l l u-n n w^{\prime}$ stammt ebenfalls vom Stamm $\chi a ̈ l l a-$. In Lehtisalos Wörterbuch erscheint das Wort 'Fisch' als ein $\ddot{a}$-Stamm: BS(K) $\chi \bar{a} l l \varepsilon, B S(\mathrm{Sj}), \mathrm{MS}$ (Oks, U-Ts.)

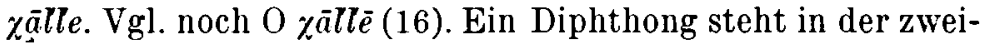
ten Silbe bei den Angaben Kan (N) $\chi \bar{a} l l e^{\bar{A}}$ und waldjur. $k \bar{a} A^{\prime} A^{\prime} \partial p$. Die diphthongische Endung und die Mouillierung des inlautenden *-l- sind überzeugende Beweise dafür, dass an den Stamm ${ }^{*} k a \bar{l} l a$ ein Suffix ${ }^{*} j \varepsilon$ getreten ist. Das Ergebnis der Verbindung ist der Lautwandel in der zweiten Silbe: ${ }^{*} a i>$ ${ }^{*} i i \sim \varepsilon i \sim e j>\varepsilon, e$. Im Selkupischen (Mat 33-34) ist das Nomen z.T. ein $\ddot{a}$-Stamm (NP Jel Tas k̨uelэ), z.T. ist es nach Abschleifung des Endvokals einsilbig geworden: kุuel, kqûel, kịl, (vgl. Pápai-Hajdú khål). Vgl. B Tas k̨uele-nnjang 'ich 
fische'. Was den Vokalismus der ersten Silbe betrifft, so ist $\bar{a}$ im Jurakischen und in Donners Angaben aus dem Jenisseisamojedischen, $\bar{o}(o)$ im Kamassischen, der Diphthong ue im Selkupischen (aber Kar $\underset{i}{i}, i$, Pápai $a$ ) und $o$ im Tawgy. (Auffallend ist der Diphthong der lappischen Wörter, wie auch lp Kld $\bar{u}$, Ter $\bar{i}$.) Den langen Laut der samojedischen Wörter können wir aus einem Diphthong am einfachsten erklären: ${ }^{*} u i \sim u e$ (selk.) $>{ }^{*}$ oi $(>\operatorname{kam} . \bar{o}, o)>{ }^{*} a i>$ jur.-jen. $\bar{a}$. Selk. $\bar{u}, \underline{\imath}$ dürften auch aus einem Diphthong stammen. Die Diphthongierung der ersten Silbe ist eine ursamojedische Erscheinung. Wahrscheinlich haben die jurakischen Stämme hale-, hali-, selk. khål den uralischen kurzen Vokal der ersten Silbe bewahrt.

'Herbstfrost; frieren, erfrieren' (vgl. Beitr. 23, 230, CompGr. $\S 242$ Note). Die nominalen und die verbalen Formen sind

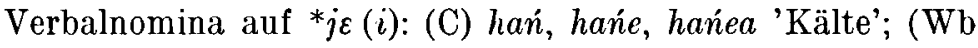

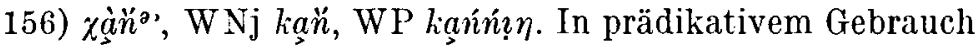
wird dem Verbalnomenstamm das verbalnomenbildende Suf-

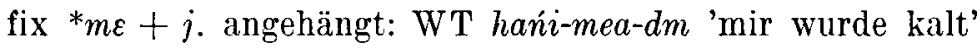

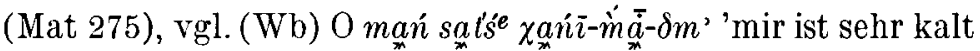

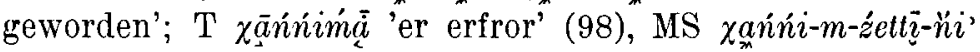
'uns (Du.) fror' (552). Ein Suffix *ta erscheint in der Form (C) han-ta-sti-mah 'ich friere' (285) und auch in den selkupischen Verbalformen N kandak, MO K NP kandang 'ich erfror' (Mat 30). Die selkupischen Nomina sind mit Ausnahme von kandek 'Herbst' Verbalnomina auf *je: 'Tas kâân-je, Kar kaanj 'Frost; starke Kälte', NP kaaji, N MO K Tsch liaai, OO keai (<*kaańe < kaan-je). Vgl. kam. (D-J) k'ånna-lam 'frieren', kinnd ${ }^{2}$-lam 'erfrieren' usw.

'Weinen; weinen' (Wb 95). Als Nomen kommt es in der Sprache der Lieder vor, manchmal als ein Parallelwort zum Nomen-Verbum mit der Bedeutung 'Stimme, Schrei; schreien': (C) seädn tjāna |jār adimeäh 'hinter dem Hügel | erschien Weinen' (218, vgl. 289, 299); MS zunnp mălengannp | tōor'

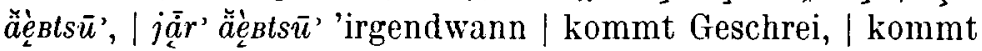
Weinen' (254). Der Kehlkopfverschluss deutet auf das geschwundene Suffix *t. : (C) jārta jär-miki $i$ 'der Weinende wohl weinte' (168); O ńe jọ̆r-mī 'das Weib weinte' (111). Das Nomen- 
Verbum kommt auch als ein mit dem Suffix *ma gebildetes

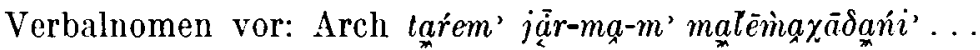
'Nachdem ich so mit meinem Weinen [eig. mein Weinen] aufgehört hatte ...' (430). In prädikativer Stellung ist das Wort ein Verbalnomen auf $* \beta+m a(+j)$ : Kan pirt'sembòi jēr $\beta$ | $j \dot{a} r \bar{u}-m m v$ 'Ziemlich grosser Fürst begann zu weinen' (234);

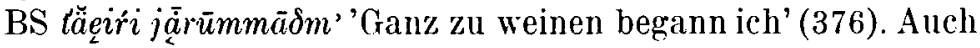
das mit dem Suffix *je gebildete Verbalnomen hat einen Zu-

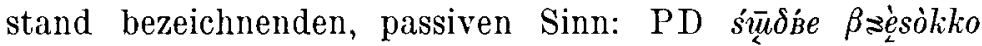
$j \bar{a} \dot{a}-j \bar{a}-\eta p ?$ 'Der Riesen-Alte weint (heftig)' (128); WNi $k \bar{a}-$ tajju jẳra-jjet 'Die Grossmutter begann zu weinen' (1+5). Dem Stamm jära- in der Angabe aus dem Ni-Dialekt entspricht der Stamm jen. jara-, jaða-, Tawgy jora-.

'Schrei; schreien' (Wb 517): (C) tor, tōor 'Stimme', tōre-si, tore-se-da 'ohne Stimme'; O t'ōr 'Ruf, Geschrei', BS (Sj) tōrr, vgl. MS (Oks) tồra-kko-m' AkkSg. Der Stamm der Verbal-

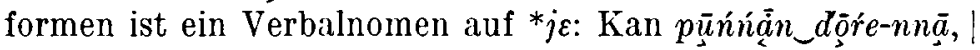
ńátto tōrennā 'er ruft nach rückwärts, | zu seinem Kameraden

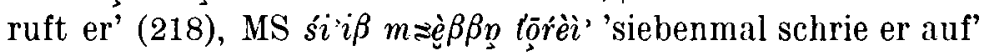
(305); Kan (M) tồr-dēe- $\beta$ 'ich rief'. Castrén führt auch ein Verb 3. Sg. tjüöriej 'er begann zu schreien' (231) an; vgl. $O$

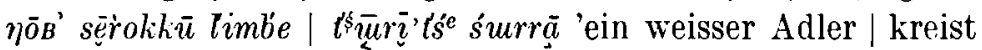
schreiend' (211). Das jurakische Nomen-Verbum hängt wahrscheinlich mit dem selkupischen Verb (Mat 99) tjuure-, tjur-, čure-, čur- 'ich schreie, weine' und mit dem kamassischen tor ${ }^{2}-, d \bar{o} r^{2}-$ 'weinen, heulen' zusammen (anders Beitr. 266). Die Zusammengehörigkeit der jurakischen und der selkup-kamassischen Stämme kann mit der Annahme erklärt werden, dass der ursprüngliche Anlaut $* t$-war, und dieser wurde durch das Vorderglied $i$ des Triphthongs oder Diphthongs der ersten Silbe palatalisiert: *ť̃oure $>>$ tōre $\sim$ türe (selk. čure,- čur-). Ein derartiger Triphthong oder Diphthong erklärt auch den jurakischen Stamm (C) tjüörie-, (L) tǚri-.

Auf Grund der lexikalischen Angaben Lehtisalos könnte man die Zahl der jurakischen Nomen-Verbum-Stämme erheblich vermehren. Für ein Nomen ohne Suffix würde man aber kaum Beispiele finden. Gewöhnlich sind beide Glieder des Wortpaars Verbalnomina, aber nicht notwendigerweise 
mit demselben Suffix gebildete Verbalnomina. Das Verbalnomen bezeichnet in prädikativer Funktion im allgemeinen einen Zustand passiven Sinns. Hier soll noch bemerkt werden, dass im Jurakischen jedes Nomen, das einen Zustand (eine Eigenschaft) bezeichnet ('Mensch'; 'jung, Kind, Junge'; 'Genosse' usw., usw.), wie ein Verb konjugiert werden kann, dass ferner in einem nominalen Satz jedes Nomen als Prädikat in der 3. P.Sg. Ind. auftreten kann.

\section{Primäre und sekundäre Verbalstämme}

Die juraksamojedischen Verbalstämme erscheinen sehr selten in ihrer ursprünglichen Form, deshalb ist ihre Gruppierung nur aus geschichtlicher Sicht möglich. Die ursamojedischen Verbalstämme waren genauso wie die Nominalstämme zweisilbig, hatten einen kurzen Vokal im Auslaut und bildeten nach dem Endvokal drei Gruppen: die ursprünglichen $a$ - und $\ddot{a}$-Stämme und die sehr grosse Anzahl der $e$-Stämme. Im Verlaufe des Lebens der Sprache traten in den jurakischen Verbalstämmen aus phonetischen Gründen erklärbare Lautwandel auf. Auf dem zweiten internationalen Kongress im Jahre 1965 in Helsinki habe ich in einem Referat die Lautwandel vorgeführt, die in drei Verbalstämmen erfolgt waren, und habe auch kurz auf die Faktoren hingewiesen, deren gemeinsame Einflüsse bei diesen und ähnlichen Lautwandeln wirksam waren (CSIFU I 482-489). Was die Gesamtheit der jurakischen Zeitwörter betrifft, so ist die bedeutende Zahl der einsilbigen Stämme auffallend. Die Hauptursache der Einsilbigkeit ist der Abfall des Endvokals des zweisilbigen Stammes. Dies war zunächst bei den $e$-Stämmen geschehen, welchem Abfall nachweislich die Reduktion des Auslautvokals des Stammes vorausgegangen war. Der zweisilbige Stamm konnte einsilbig werden durch den Ausfall des ursprünglichen inlautenden Konsonanten bzw. in gewissen Fällen durch die Vokalisation der Semivokale. In den zweisilbigen Stämmen lässt sich der enge Zusammenhang, die gegenseitige Wechselwirkung der beiden Silben beobachten. Auch zwischen dem 
Konsonantismus und dem Vokalismus ist ein enger Zusammenhang zu erkennen. In den anlautenden Konsonanten und mittelbar im System des Konsonantismus hat z.B. das kurze $i$, als erstes Element eines Diphthongs oder Triphthongs der (ursprünglichen) ersten Silbe, tiefgreifende Wandel herbeigeführt.

Die in den urjurakischen Verbalstämmen erfolgten Lautwandel können — wenn die Möglichkeit dazı gegeben ist -.. auf Grund eines Vergleiches mit den entsprechenden Verbalstämmen der übrigen samojedischen Sprachen verstanden werden. Forschungen auf diese Weise führen zur Erkennung der aus primären Stämmen stammenden sekundärenVerbalstämme. In diesen wird dem primären Stamm, der oft gar nicht gebraucht wird, ein Ableitungssuffix angehängt. Die Funktion des Suffixes ist verblasst. In anderen Fällen lebt auch der primäre Stamm, doch ist sein Zusammenhang mit dem sekundären Stamm derart locker geworden, dass die $\mathrm{Zu}$ sammengehörigkeit der zwei Stämme erst bewiesen werden muss.

Die Lautwandel der zweiten Silbe sind von der Qualität des Auslautvokals der zweiten Silbe abhängig und nicht zuletzt davon, ob sich z.B. das den Lautwandel herbeiführende Suffix mit dem ursprünglichen Auslautvokal oder mit dem konsonantischen Stamm verbindet. Hinter der zweiten Silbe ist wahrscheinlich die lange Quantität sämtlicher Vokale etymologisch, im Falle von $\bar{o}, \bar{u}$ muss man aber der velarisierenden und verlängernden Wirkung des folgenden $l(=l)$ und $r$ Rechnung tragen.

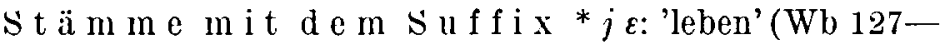
128). Fi. elä-, ung. él (vgl. éle-k 'ich lebe', éle-t 'Leben') hat bekannte Entsprechungen in allen samojedischen Sprachen. Der Endvokal * $\ddot{a}$ wurde bewahrt in der Tas-Mundart des Selkupischen und in einigen Angaben aus N: ele-śpak, sle-spak (Mat 21). Der Anlautrokal ist $e,(\varepsilon)$ in $N$ und zum Teil in $\mathrm{MO}$, in anderen Mundarten $i$, in einem Verbalnomen aus OO $i i(\bar{i})$. Gerade auf Grund des Stammes iil-können wir es für wahrscheinlich halten, dass der Lautwandel $e>i, i$ durch einen 
geschwundenen anorganischen Anlaut $j$-verursacht wurde. Dieses $j$-erscheint in allen jurakischen Dialekten: tundrajur.

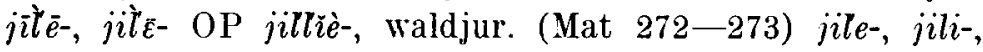
B jire-, jiri-, (Wb) jirrí-, jirŕri-. Alle diese Stämme sind mit dem Suffix ${ }^{*} \varepsilon \varepsilon$ gebildete Verbalnomina, auch die Mouillierung des *-l- im Inlaut hat das Suffix verursacht. Die eigentliche Bedeutung des Wortes (Wb 126) O Sj jil', OP jil' 'Lebenszeit' ist 'Leben'. Der Kehlkopfverschluss deutet auf ein abgeschliffenes verbalnomenbildendes Suffix * $t$ (vgl. C $j \bar{a} r$-ta 'Weinen'), das auch im ungarischen Wort élet vorhanden ist. - Das anlautende $j$ - ist verschwunden in einigen Angaben von Reguly: saua-ile, -ile 'reich' [gut lebend], voj-ile 'arm' [schlecht lebend], wo das Glied ile seine ursprüngliche Bedeutung als Verbalnomen bewahrt hat. ${ }^{1}$

'sterben' (Wb 174). Die bekannten samojedischen Entsprechungen des finnischen Verbs kuole-, ung. hal- sind alle einsilbig: jur. $\chi \bar{a}-, k \bar{a}$ - (vgl. Sprogis Kan $h a$ 'gestorben, tot, krepiert'); jen. $k \bar{a}-;$ Tawgy $k \bar{u}-;$ selk. (Mat. 38-39) ku, K Tsch OO kiu-, kam. $(\mathrm{D}-\mathrm{J}) \hat{k} u-$. Dem ursamojedischen Stamm *küll(e) wurde das verbalnomenbildende Suffix ${ }^{*} j \varepsilon$ angehängt: ${ }^{*} k \bar{u} l+j .>>$ ${ }^{*} k \bar{u} l->{ }^{*} k \bar{u} i->k \bar{u}-\sim k u$-. (Im Kamassischen $u>u$ unter dem palatalisierenden Einfluss von ${ }^{*} i$.) Anders war die Entwicklung im Jurakischen und im Jenisseischen gerichtet: aus dem Stamm ${ }^{*} k \bar{u} i$ - wurde ${ }^{*} k \bar{o} \bar{i}-$, daraus ${ }^{*} k \bar{a} \dot{i}$, später $\bar{a}$, wie auch in einigen anderen Fällen. Der waldjurakische Stamm Kis $k a \tilde{a} \dot{e}-$ lässt sich aus einer Form mit dem Diphthong ${ }^{*} \bar{a} i>$ * ài $>\check{a} e ̨$ erklären (anders CompGr. $§ 199$ ).

'kommen' (Wb 493-494). Die Entsprechungen des finnischen Stammes tule- sind Einsilber. In einer bedeutenden Gruppe der jurakischen Verba und Verbalnomina erscheint $t_{\bar{o}}$ - (in einzelnen Angaben to-), in einer kleineren Gruppe $t \bar{u}-$ $(t u-)$. Auffallend ist der Stamm WLj $t u^{2}$, der dem Stamm tui- einiger Verbalformen im Tawgy entspricht. Aus dem ursamojedischen Verbalnomenstamm *tul $+j$ entwickelte sich

1 Interessant ist, dass die kolalappischen Entsprechungen in der ersten Silbe den anorganischen Laut $j$ - enthalten und dass dem langen $i$ des jur. $j i l$-, selk. $i l$ - ein Diphthong (bzw. $a ̈$ ) entspricht: (T. I. Itkonen Wb 59) jëäla-m (jä-) usw. 'ich lebe'. 
${ }^{*} t u l-$, aus diesem teils *tui (vgl. Tawgy, WS), teils *toi-, deren Fortsetzungen die jurakischen Stämme $t \bar{u}-, \bar{o}^{-}$sind. Im Kamassischen (Gr. S. 545) existiert die (unter den lexikalischen Angaben nicht vorhandene) Entsprechung des finnischen Verbs tule-: "tu-lam ich komme, Imperativ $t u^{\prime}$, der Stamm $t u-»$. (Der in der sprachwissenschaftlichen Literatur als traditionelle, fragwürdige Entsprechung des finnischen Verbs angesehene kamassische Stamm š- gehört also nicht hierher.)

'ansehen, betrachten' (Wb 245). In den Angaben des Wörterbuches und der Texte von Lehtisalo erscheinen die Stämme mannē-, manne-, manna-, mà̀-, im Waldjurakischen manne-, manni-, mannäèe-. In Castréns Texten finden sich die Stämme mane-, man- $(2,82)$ und mana- $(246,274)$, manna- (240) und man̄- (272). Der lange Endvokal des Stammes manā- ist aus der Verbindung des Auslautvokals des Stammes mana- mit dem Suffix ${ }^{*} j \varepsilon(i)$ entstanden. Das Ableitungssuffix ${ }^{*} j \varepsilon$ ist in Castréns waldjurakischen Angaben enthalten: mana-je$n g a-u$ 'ich sehe, betrachte' (vgl. mana-je-ngu-u) (Mat 279). In den Paradigmen von Sprogis wird das Suffix $* j \varepsilon$ dem konsonantischen Stamm angehängt: man-je-m, manjen, manje usw. (ALH II, 172). Es ist klar, dass die Vokale $\bar{e}, e, i, \underset{a ̈ e}{e}$ in Lehtisalos Angaben sekundäre Laute sind, die aus der Verbindung des Auslautvokals des Stammes mana- mit dem Suffix ${ }^{*} j \varepsilon$ entstanden sind (vgl. AblSuff 75). In Castréns Texten findet sich auch der Stamm manji- (205), bei Lehtisalo mañii- (3), mañi- (446). Alle diese Stämme stammen aus der Verbindung ${ }^{*} \operatorname{man}(e)+j \varepsilon$. (In den entsprechenden selkupischen Stämmen [Mat 56] mandśe-, mandśa-, manne-, manna- und im kamassischen mande- gehört das Element dśe, dśa, de nicht zum Stamm.)

St äm m e m it dem Suffix *ka (*kal): 'legen, laden' (Wb 351). Das jurakische Zeitwort, das zur Wortfamilie des finnischen Verbs pane- gehört, erscheint in Castréns Paradigmen als ein Verb mit $\eta$-Stamm (Gr. S. 413-416), die Imperativform der 2 . Sg. ist aber puena-d! 'lege es!'; vgl. noch puen-s 'legen'. In Castréns Texten kommen noch die Verbformen puen-ga-da $(81,261)$, puen-ga-haju-da (22) vor. Unter 
Lehtisalos Angaben aus dem O-Dialekt findet sich der Stamm

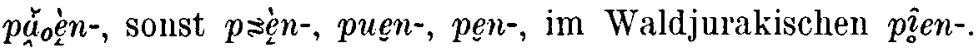
Auch für den Stamm vom Typ puena- gibt es vereinzelte Angaben aus Lehtisalos Texten: Arch $p \approx \bar{e} \eta \bar{a}-\beta$ 'ich tat (den

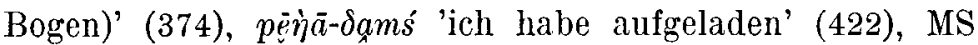
$p \approx e \bar{\eta} \bar{a}-\beta \beta p^{\prime}$ ' 'wir beladen' (554). Bei der Feststellung des Lautwertes des - $\eta$ - ist Paasonens Bemerkung zu beachten: "In Castréns Handschriften scheint das Zeichen $\eta$ nach Vokalen überhaupt nicht vorzukommen, sondern statt dessen $n g$, resp. $n k$. An einer Stelle heisst es, dass $n g$ und $n j$ 'einen so tiefen nasalen Ton annehmen, dass sie fast in einen einzigen Laut zusammenschmelzen und vielleicht eigentlich mit einem einfachen Zeichen zu bezeichnen wären'» (Beitr. 33). -- Ohne Zweifel ist das jurakische pue $a^{-}$ein sekundärer Stamm aus ${ }^{*}$ puen $+k a$. In den selkupischen Zeitwörtern erscheinen einsilbige Stämme mit $n$ am Ende: $\mathrm{N}$ pan-nap, Tsch pän-dam, Tas pin-bam 'ich legte es' usw. (Mat 71, 200, 209). Donner' (Anl lab 171) hat auch die Formen Ty pan-nā , KeO $p^{\ddot{n} n-n a m}$ mitgeteilt. Die grosse Mannigfaltigkeit der Vokale der ersten Silbe im samojedischen Wort lässt sich vielleicht mit der Annahme begründen, dass es im Ursamojedischen neben ${ }^{*} \dot{a} \sim \ddot{a} \sim e$ auch Diphthonge wie ${ }^{*} \dot{a} i \sim \ddot{a} i \sim e i$ bzw. ${ }^{*} \dot{a} e$, * äe gegeben hat. Die Entstehung der Diphthonge vom jurakischen Typ ue hängt mit der labialisierenden und velarisierenden Wirkung des Anlautes $p$-zusammen und zugleich mit der Ausbildung des Lautsystems, das sich in Lehtisalos Texten und lexikalischen Angaben widerspiegelt. Von den Eigentümlichkeiten dieses Lautsystems hat Bo Wickman eine eingehende und klare Beschreibung gegeben (FUF XXXIII, 96--130). Einige Erscheinungen des Velarisationsprozesses lassen sich auch in Castréns Texten beobachten. In Castréns Material finden wir auch Angaben, die z.B. die Herausbildung der Doppelheit der anlautenden $p--\dot{p}$ - beleuchten. In Castréns waldjurakischen Aufzeichnungen steht das Wort mit der Bedeutung 'Geschwür' piungos (Mat 238). In Lehtisalos Wörterbuch (389) erscheint dasselbe Wort als O ṕunkapt's, $\mathrm{Nj} \dot{p} i \eta k_{B} t_{s}$ 'Blutbeule, Geschwür'. Die Mouillierung des $p$ - und den Lautwandel $u>\ddot{u}$ hat das $i$-Element des Diph- 
thongs herbeigeführt: $\breve{\imath}$ ist im anlautenden $* p$ - aufgegangen. Da die Velarisation gewisser palataler Vokale in Zusammenhang mit dem Schicksal gewisser anlautender Konsonanten zu stehen scheint, kann es für wahrscheinlich gehalten werden, dass die Bildungsstelle dieser Konsonanten mehr nach hinten rerschoben wurde, dass also die Artikulationsbasis im Jurakischen sich infolge bisher noch unbekannter Faktoren verändert hat. Eine solche Verschiebung ist auch im Ungarischen geschehen, in entgegengesetzter Richtung (vgl. Gíza Bírczi: A magyar nyelv iletrajza [Die Lebensgeschichte der ungarischen Sprache] S. 141). -.- Auch das zur Wortfamilie von fi. puno-, ung. fon gehörende (C) panal- (Gr. +16-419) und das in Lehtisalos Wb (342) registrierte Zeitwort $\mathrm{O}$

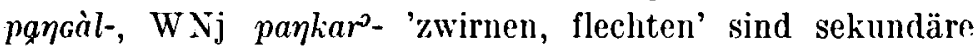
Stämme, wo an den Stamm pan-das Suffix $k a+l$ angehängt ist (vgl. Mat 286, 316). Unter den selkupischen Entsprechungen ist auch dieser sekundïre Stamm (Mat 71) zu sehen: K pan-gal-pau 'ich zwirnte', pan-gan-nau id. Das Element $k a$ und $l$ sind verbalnomenbildende Suffixe mit inchoativer Frundfunktion.

s't a m m m it de m suffix *ma: 'schieben (z.B. ein Boot ins Wasser)' (Wb 283--284). Stammvarianten o $\mathrm{T}$

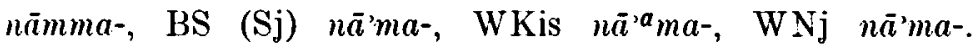
(Die gleichen Stämme erscheinen iluch in den Stammformen, die ein intensiv-effektives Suffix $r$ enthalten.) In einigen Verben wird an den sekundären Stamm vom Typ nā'ma-das Suffix $l$ angehängt, und aus dieser Verbindung entstehen die Stämme BS nā' $\beta o l-$, Kan (Sjo) nä'ul-, vgl. (C) näuol-mīodo' 'sie schoben sie' (265), naol-jiedo' 'sie stossen sie' (267) usw. Es ist nicht klar, was in den mit $l$ gebildeten Stämmen den Lautwandel $m>\beta$ verursacht hat. - - Auch die Frage soll beantwortet werden, welchen Konsonanten der Kehlkopfverschluss in den Stämmen vom Typ $n \bar{a} ' m a-$ vertritt. Das selkupische Verb mit der Bedeutung 'ich stosse' zieht unsere Aufmerksamkeit auf sich (Mat 64); von seinen verschiedene Suffixe enthaltenden Formen können folgende Stämme abgetrennt werden: B Tas Kar nokia-, Tsch noogo-, Tsch OO 
noyo-, Tschl nogo-. Auf Grund des jurakischen $\bar{a}$ und des selkupischen $\bar{o}$ in der ersten Silbe kann angenommen werden, dass der primäre ursamojedische Stamm *nōka (*noika) oder *nōke (*noike) lautete. Im Suffix ma und in den sekundären Stämmen derartigen Typs können wir das urtümliche verbalnomenbildende Suffix ${ }^{*} m a$ erkennen.

St a m m m it dem Suffix *r.: 'zusammenfahren; erschrecken' (Wb 224). Im Waldjurakischen kennen wir die

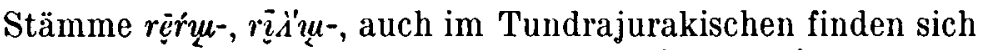

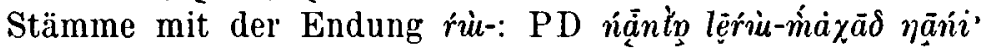
lẹriü-settī 'nach dem Zucken seines Kameraden erbebt auch er' (118); vgl. (C) seäinda leärjo-s 'erschreckt' (287); L läècoō 'erschreckend' (206). In allen diesen Stämmen gehört das Element $r$ nicht zum Stamm, sondern es ist ein intensiv-effektives Suffix. In Castréns Wörterbuch finden sich auch andersartige Stämme: lied-rie-, led-rie-, led-ri-ıla-, led-ri-ta-'zittern'. Das Zeitwort wurde in ähnlicher Form und Bedeutung auch von Reguly aufgezeichnet: leäd-riä-. In Lehtisalos Texten kommt auch

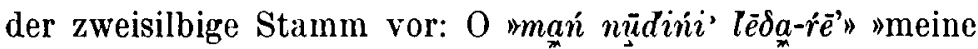
Hände zittern" (3). Das Suffix 'iē lässt sich aus der Verbindung $*_{r \varepsilon}+j \varepsilon$ erklären. In den jurakischen Verbalstämmen ist das inlautende $*_{-} \delta$ - - mit Ausnahme dieser wenigen Angaben - ausgefallen. Unter Berücksichtigung sämtlicher Angaben erscheinen ror dem Suffix * $r \varepsilon$ die folgenden primären Stammvarianten: (L) lè $\delta a-;$ (R) leäd-, (C) lied-, led-, lé-, le-, v'gl.

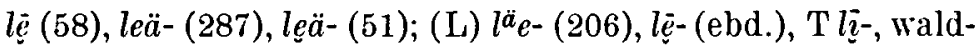
jur. re $\bar{e}^{-}, r i-$ - - Im Selkupischen, Kamassischen und in den nordsamojedischen Sprachen hat der durch Ausfall des *- $\delta$ - entstandene einsilbige, mit dem Suffix $r$ gebildete Stamm Entsprechungen: selk. (Mat 54-55) N ler-, ler-, lar-, in den anderen Mundarten neben ler-, ler-, 00 lär-, 00 Tsch när-; kam. (DJ) ne-re-, nere-. Aus diesen Stämmen sind Verba mit der Bedeutung 'ich fürchte mich' entstanden. - Die jurakischen Stämme mit der Endung ró- und r $r u$ - haben auch im Selkupischen passivische Entsprechungen: MO K le-ru-ang, OO läruang, Tsch näruang 'ich crschrak' (eig. ich wurde erschreckt). Interessant ist, dass im Jenisseischen und im Tawgy, die dem Jurakischen nahe stehen, aus dem durch 
den Ausfall von *- $\delta$ - entstandenen einsilbigen Stamm mit dem verbalnomenbildenden Suffix ${ }^{*} m a$, bzw. ${ }^{*} m \varepsilon(+j)$ Stämme mit passiver Bedeutung entstanden: Jen. lume-, lumi-, Tawgy lu-ma-. Der Vokalismus der ersten Silbe ist im samojedischen Verb sehr mannigfaltig. Unter Beachtung des mouillierten anlautenden $l$ - können wir von einem ursamojedischen Diph-

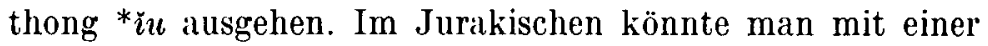
Entwicklung in zwei Richtungen rechnen: 1 . * ${ }^{2} u>*_{\imath o}>$

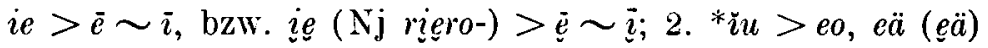
$\sim \ddot{a}_{e}$. Im 'Tawgy ${ }^{*} l \grave{\imath} u>l u-$. Im Jenisseisamojedischen erfolgte - wie in vielen anderen Fällen - Monophthongierung.

Das einseitige Material mit begrenztem Umfang ist freilich nicht geeignet, vom System des urjurakischen bzw. ursamojedischen Vokalismus ein Bild zu geben. Was aber die Quantitätsverhältnisse der ersten Silbe betrifft, unterliegt es kaum einem Zweifel, dass die erste Silbe neben kurzen Vokalen auch Vokale von langer Quantität enthielt. In den Begriff der langen Vokale gehören auch die ursamojedischen Diphthonge und Triphthonge hinein, dies bedeutet aber nicht, dass in den samojedischen Sprachen alle langen Vokale der ersten Silbe aus Diphthongen oder Triphthongen stammen würden, obwohl eine solche Lautentwicklung in vielen Fällen nachgewiesen werden kann. Beachtung verdienen solche Stämme, bei denen dem finnischen kurzen Vokal in der ersten Silbe im Ursamojedischen ein langer entspricht. Es erscheint sicher, dass die Laute $\ddot{o}-\overline{\tilde{o}}, \ddot{u}-\overline{\tilde{u}}, \varrho-\bar{e}, \underline{i}-\vec{\imath}$ im Selkupischen sekundäre Laute sind, genauso wie im Kamassischen und Jurakischen; diese Laute können also nicht zum ursamojedischen Vokalsystem gehört haben. weder in der ersten noch in der zweiten Silbe.

\section{Zum juralsamojedischen Konjugationssystem ${ }^{1}$}

Wenn wir das System der juraksamojedischen Konjuga-

1 Péter Hajdú hat seine Ansichten über die samojedischen Modi, die temporalen Verhältnisse und die Personalsuffixe in seinem Werk $\triangleq B e-$ vezetés az uráli nyelvtudományba" (Einführung in die uralische Sprachwissenschaft. Budapest, 1967) S. $71-78,136-144$ ausgeführt, mit Hinweisen auf die Literatur. 
tion nicht auf Grund der Grammatiken, sondern auf Grund der Texte Castréns und Lehtisalos studieren, können wir feststellen, dass zwischen den Konjugationen der juraksamojedischen und der finnisch-ugrischen Sprachen grosse Unterschiede bestehen. Die jurakische Konjugation gehört mit Ausnahme der Personalsuffixe wie auch der die Dualität und Pluralität bezeichnenden Elemente eigentlich in die Ableitungslehre. Diese Anschauungsweise bestimmt die Methode der Erforschung der jurakischen Verba. Um eine Zeitwortform und den eine solche Zeitwortform enthaltenden Sat\% genau auszudeuten, genügt es nicht, die Zeitwortform allein zu analysieren. Selbst dann wird kein angemessenes Resultat erzielt, wenn die Verbform im Rahmen des Satzes untersucht wird. Das Wesen der juraksamojedischen Konjugation kann nur in einem zusammenhängenden Text erkannt werlen.

Im Jurakischen gibt es zahlreiche Verbformen, in denen die grosse Zahl der Ableitungssuffixe zwischen Stamm und Personalsuffix auffällt: 0 "pádàr jảa $m a \chi \bar{a} n \grave{t} \chi \bar{a} n n \bar{o}-n-t_{x} a-s^{E}-t t a-\eta \eta \bar{u}-\delta-m$ ') "wenn ich deine Spuren treffe, we rde i c h deinen Spuren op fern (Blutopfer)" (51). Der Stamm $\chi \bar{a} n n \bar{o}-$ ist an sich ein sekundärer Stamm mit dem Suffix $* \beta$, jedes cinzelne Element zwischen dem Stamm und dem Px. der 1. Sg. hat seine Bestimmung. Die Häufung von Ableitungssuffixen ist keine gehaltlose Formalität in den jurakisclien Verben diesen Typs. Die Suffixe sind organische Teile der Verbform, und die Bedeutung der Verbform setzt sich aus der Bedeutung der Suffixe zusammen. Die Bedeutung solcher Verbformen kann gar nicht genau übersetzt, sondern nur mehr oder weniger annähernd umschrieben werden. Die feinen Funktionsnuancen, die die Suffixe bezeichnen, können nicht summiert, in einer Einheit verschmolzen werden in einer Sprache, in der sich die Kategorien der Konjugation, die Jodus- und Tempuszeichen herausgebildet haben. Im Jurakischen gibt es nur nach den Grammatiken Tempora, und die Forscher können auch nur mit finnischugrischer Anschauungsweise Tempora im Jurakischen erkennen. Auch Modi gibt es nicht in dem Sinne wie in den finnischugrischen Sprachen, denn es gibt ja keine Moduszeichen, deren 
einzige Funktion die Bezeichnung eines gewissen Modus wäre. In Verbformen, in denen eine Häufung von Ableitungssuffixen erscheint, ist sogar die Stelle der sog. Moduszeichen, d.h. die Reihenfolge der Suffixe nicht unbedingt dieselbe. Die sprachliche Eigenart, die die Verbformen dieses Typs widerspiegeln, erinnert in struktureller Hinsicht auffallend an den sprachlichen Zustand, den die tungusischen Zeitwörter vertreten (vgl. H. Winkler: JSFOu XXX,9 18-19, 22-23).

Das juraksamojedische Konjugationssystem ist aufgebaut auf der strengen, konsequenten, mit sprachlichen Elementen bezeichneten Unterscheidung von Realität (Wahrnehmung, unmittelbare Erfahrung, tatsächliche oder vermeintliche Wirklichkeit, Gewissheit) und von Irrealität in einem gewissen engeren Sinne (von anderen gehörte, in der Phantasie erlebte, von Bedingungen abhängige, einen Wunsch oder eine Absicht ausdrückende, in der Zukunft mögliche, mit einem Wort: ungewisse Geschehnisse). Aus jurakischer Sicht ist es nicht überraschend und nicht unbegründet, dass in Fragen, in der Verneinung, und auch in den Ich-Liedern Ableitungssuffixe auftreten, die dic Irrealität bezeichnen. Der Gesang, den Ego vorträgt, ist nur eine traditionelle Vortragsform, der Sänger erzählt oder singt Geschehnisse, die er von anderen gehört hat. Wie sehr sich im Juraksamojedischen die Bezeichnung des Konkreten rom Nichtkonkreten unterscheidet, beweist die "Selbstbiographie", in der Lehtisalo von einer Frau die Ereignisse ihres Lebens, also persönliche Erlebnisse erzählt bekam (Mat 347--351). In ihrer Mitteilung gebraucht sie neben nicht sehr häufigen Aoristformen (die auch als Konjunktivformen gedeutet werden können,) auch das Gerundium und solche Verbformen, in denen die Suffixe teils Passivität, teils Irrealität ausdrücken. Passivische und die Irrealität bezeichnende Verbformen erscheinen auch in den Beschreibungen, in denen die Gewährsleute Lehtisalo die Lebensweise der Juraksamojeden, aus eigener Erfahrung wohlbekannte Gewohnheiten (Zeltbau, Fuchs-, Seehundjagd usw.), nicht aber spontane Geschehnisse erzählten (vgl. die entsprechenden jurakischen Texte der Materialien).

Die Realität, die Gewissheit wird durch den Indikativ ver- 
treten, d.h. durch den Verbalnomenstamm auf $* j \varepsilon$ und dessen Formen aktiver Bedeutung mit Hinweisen auf die Person. Die Aoristformen des Indikativs sind achronistisch: sie drücken nur das Geschehene aus, ohne Zeitbezug. ${ }^{1}$

Das Fachwort "Geschehnis" entnehme ich einer umfangreichen Studie des scharfsinnigen und gelehrten Altphilologen Gyula Gyomlay. Diese Studie, die unter dem Titel »Az úgynevezett igeidốk elmélete» [Die Theorie der sogenannten Tempora] in den Nyelvtudományi Közlemények (XXXVIIXLII) erschienen ist, wäre in der internationalen allgemeinen Sprachwissenschaft sicher ein oft zitiertes Werk gewesen, wenn sie nicht in ungarischer Sprache geschrieben worden wäre. Gyomlay analysierte zunächst auf Grund einer tiefschürfenden Untersuchung des altgriechischen Sprachmaterials mit strenger Logik die Lehrsätze der Grammatiker, er wies auf die Irrtümer hin und bestimmte gewisse Begriffe, die auch bei der Untersuchung der Zeitverhältnisse und der Modi in den uralischen Sprachen aufschlussreich sein können, zumal die Verknüpfung der Aktionsart und der temporalen Bezüge, ein Erbe der griechisch-lateinischen Grammatiker, auch bei der Untersuchung der temporalen Verhältnisse in den uralischen Sprachen eine bedeutende Rolle gespielt hat.

In seiner erwähnten Studie fand Gyomlay es zweckmässiger, anstatt der Benennung "Tätigkeit" die Benennung "Geschehnis" zu brauchen, weil dieser weiter gefasste Ausdruck auch die passiven Formen einbezieht. In die Benennung Geschehnis zog er auch den mit dem Geschelınis verbundenen, bzw. infolge des Geschehnisses entstandenen Zustand hinein. Die Zeitform des Verbs (chronos, tempus, Zeitstufe) ist der Ausdruck eines subjektiven Verhältnisses. Der Sprecher be-

${ }^{1}$ In den jurakischen Indikativformen hat die Verschmelzung des Suffixes * $j \varepsilon$ (i) mit dem Stammauslautvokal die lange Quantităt des Vokals in der zweiten Silbe des Stammes herbeigeführt. Es lãsst sich schwer entscheiden, $o b$ in Castréns Paradigmen das Personalsuffix der 1. und 2. Person in den Formen madam, madan usw. an den primāren Verbalstamm gehăngt ist, oder ob wir es bei diesen Formen mit einer sekundären Kürzung zu tun haben. In Lehtisalos Texten ist der Vokal in der zweiten Silbe der Indikativstämme in der Regel von langer Quantität. 
zieht seinen Standpunkt zum Nacheinander der Geschehnisse auf den objektiven Inhalt der Wortformen, die das Geschehnis bezeichnen. Vom Gesichtspunkt des Sprechers aus kann man die Vergangenheit (praeteritum), die Gegenwart (praesens) und die Zukunft (futurum) mit Verbformen ausdrücken. Die Zeitformen, die die Tradition - irrtümlich — tempora nennt, bezeichnen zumeist überhaupt keine Zeitstufe. In den älteren Perioden der Entwicklung der Kultursprachen finden wir nur eine Zeitstufe, die mit einer einfachen Verbform ausgedrückt werden kann, nämlich die Vergangenheit (praeteritum), aber auch diese bezeichnet eigentlich keine Zeitstufe (NyK XXXVII, 82). ${ }^{1}$ Präsensformen sind nichts anderes als Nicht-Präteritumformen, d.h. als achronistische, die Zeitstufe nicht bezeichnende Formen (ebd.). Bezüglich der Modi stellt Gyomlay fest: "Der Imperativ, der Konjunktiv und der Optativ . . können nach dem Wesen dieser Modi nie etwas anderes bedeuten als einen Zustand in der Zukunft, und ebendeshalb können sie bei Homer nirgends, nur in der Rede der Personen vorkommen" (ebd. 92), aber auch diese drücken keine Zeitstufe aus, sie sind achronistisch (ebd. 196).

Der in den klassischen Sprachen geschulte Castrén gebrauchte in seiner Grammatik die tempus bezeichnenden Ausdrücke der Grammatiker nicht; er deutete mit dem Fachausdruck "erste, zweite, dritte Zeit" an, dass die Fachwörter der Grammatik zum Ausdruck der temporalen Verhältnisse in den samojedischen Sprachen nicht geeignet sind. Auch Setälä hat in seinem Werk von tiefer Wirkung "Zur Modus- und Tempusstammbildung in den finnisch-ugrischen Sprachen" (JSFOu II, 1887) erklärt, dass "Aktionsart" und "Zeitstufe» nicht identische Begriffe sind: *auch aus dem Gebrauch der Tempora ersieht man, dass sie eigentlich keine Zeit bezeichnen, zu deren Angabe sie auch kein Element erhalten" (S. 174). Johannes Angere knüpft im Kapitel "Die Tempussuffixe" (S. 106 --110) seines Werkes "Die uralo-jukagirische Frage» (Uppsala 1956) sehr interessante und lehrreiche kritische Bemerkungen

${ }^{1}$ Auch Paavo Ravila betont die Priorität des Präteritums gegenüber dem Präsens (CIFU 70-72). 
an die Deutungen des Aorists ( $=\mathrm{C}$ »erste Zeit») und des mit dem Zeichen $s$ ausgedrückten Präteritums (=C »zweite Zeit») in den samojedischen Sprachen (S. 110): "[N.M.] Tereščenko selbst betont, dass man im Jurakischen oft mit Vorbehalt von Tempus sprechen kann, und sie bedient sich des Ausdruckes "Zeit" nur aus praktischen Gründen, um denen zu helfen, die sich in ihrer Muttersprache an die Kategorie der Zeitstufe gewöhnt haben (KONY S. 371)."

Um die juraksamojedischen Modi zu verstehen, soll Castréns Feststellung in Betracht gezogen werden: "Eigentlich setzt im Samojedischen jeder Modus, jedes Tempus und mit cinem Worte jede Verbform cinen besondern Stamm oder eine Grundform voraus, die ihrer eigentlichen Natur nach ein Nomen verbale ist..."(Gr. $\$ 465$, vgl. $\S 464)$. Auch Ravila betont, dass die Modusstämme in den uralischen Sprachen ohnr Ausnahme Verbalnomenstämme sind (KUUS, 63).

Diese Zitate und Bemerkungen bestimmen die Gesichtspunkte, unter deren Beachtung ich einige Fragen der jurakischen Konjugation behandle. Die Untersuchung der juraksamojedischen Verba auf Grund der Texte bestätigt die Auffassung Ravilas: "Es kann kein Zweifel darüber herrschen, dass das Aufkommen von Konjugation in den samojedischen und finnisch-ugrischen Sprachen von dem Gesichtspunkt erklärt werden muss, dass das Verb -- wenigstens für unser heutiges Sprachgefühl -..- als ein Nomen aufufassen ist" (KUUS S. 60). (Zitiert aus Angeres erwähntem Werk s. 114. Vgl. noch Paavo Ravila: "Die Wortklassen, mit besonderer Berücksichtigung der malischen Sprachen", JSFO॥ $59,3 . \$$ - 13.)

Das Verhältnis der subjektiven Konjugation zur objektiven ${ }^{\perp}$

Es ist aus Castréns Grammatik bekannt geworden, dass es in den nordsamojedischen Sprachen - ebenso wie in einigen

${ }^{1}$ Die Erscheinungsformen des Objekts im Juraksamojedischen und in den uralischen Sprachen im allgemeinen hat Bo Wickman in seinem Werk "The Form of the Object in the Uralic Languages" (Uppsala 1955) ausführlich behandelt. Mit diesem Werk beschäftigen sich die Besprechungen von A. J. Joki (FUF XXXII, Anz. 1-41) und von Péter 
finnisch-ugrischen Sprachen (Ungarisch, Obugrisch, Mordwinisch) - einen Unterschied gibt zwischen subjektiver und objektiver Konjugation. In der Grammatik (S. 377) lesen wir die folgende, auch auf das Selkupische und das Kamassische bezogene Bemerkung: "Besonders muss man in allen Dialekten [ = Sprachen] einen genauen Unterschied zwischen den transitiven und intransitiven Zeitwörtern nicht nur ihrer verschiedenen Bedeutung, sondern auch wegen ihrer verschiedenen Flexion machen.» ${ }^{1}$

Castrén stellt fest, dass die selkupische und die kamassische Konjugation eine gewisse Verarmung und Vereinfachung gegenüber dem komplizierten Konjugationssystem der nordsamojedischen Sprachen aufweiset. Er bemerkt ausserdem, dass im Selkupischen - ähnlich wie im Kamassischen - eine gewisse Neigung wahrzunehmen ist, "die transitive oder intransitive Natur des Verbums ganz und gar den Gebrauch der Personalaffixe bestimmen zu lassen” (Gr. § 539). Bezüglich des Selkupischen bemerkt Joki: "Nun ist aber die Grenze zwischen subjektiver und objektiver Konjugation bzw. zwischen den intransitiven und transitiven Verben relativ schwankend...")(CSIFU I 224).

Hinsichtlich der jurakischen Konjugation sind die possessiven Personalsuffixe aufschlussreich, die in der auf einen Besitz bezüglichen Nominativform des selkupischen Wortes loga 'Fuchs' erscheinen (Mat 152-168). Zu beachten ist, dass es aus den $\mathrm{N}$ - und Ket-Dialekten auch für die Formen Akk. + Px. Sg., Du., Pl. Angaben gibt. Im Dialekt Ket ist Nom. = Akk.: Sg. $u$; Du. wi; Pl. $u t$; im Dialekt N: Sg. Nom. = Akk.

Hajdú (NyK LIX, 247-251), ferner des letzteren Artikel "The Form of the Object in Forest Yurak" (ALH X, 95-115). Mit dem Problem der Herausbildung der objektiven Konjugation haben sich unlängst Károly Rédei - mit Hinweis auch auf die frühere Literatur - (MNy LVIII, 421-435), ferner Tibor Mikola (MNy LXI, 441-461) befasst. Letzterer hat auch seinen Standpunkt zu einigen Fragen der samojedischen Konjugation dargelegt. Vgl. noch Matti Liimola (CSIFU I 313318) und P. Hajdú: Bevezetés (75-77).

1 Es erübrigt sich hier, für die objektive Konjugation Beispielsätze anzuführen, es gibt deren in grosser Zahl bei Bo Wickman (a.a.O. $74-$ 108) und P. Hajdú. 
$m \sim p$; Du. Nom. $i, w i$, Akk. $m i, w i$; Pl. ut. Die Nominativ + Px. Formen der übrigen Dialekte: 1. Pers. Tschl OO $u ; u$; $u t$; NP Tas Kar B $m$; mi; men (B auch met). - 2. Pers: $l$; $l i$; lat, lt; Tas B len (B auch let); Tschl OO lta, ltä. - 3. Pers.: $t, d ; t i, d i ; d a t, d a ̈ t, d e t$, Tas den. Auch die Endungen sind lehrreich, die in den Indikativformen des transitiven Verbs mit der Bedeutung 'anzünden' erscheinen (Mat 196-226, vgl. Jel 216, Tas 217, B 223-224): 1. Sg. N $p$; NP Tschl Tas Kar B $m$; MO Ket $u ; 1$. Du. N MO Tschl Tas B $i$, NP $o$, Ket $\bar{o}, i$; 1. Pl. N MO Tschl $u t$; Ket $u t \sim o t ;$ NP ot, Tas B men. - 2 . Sg. $l$; Du. $l i$; Pl.N let, NP lat, lat, MO Ket $l t$, Tschl lät, lt, ltta, lttä, Tas, B len. - 3. Sg. $t, d$; Du. $t i, d i, \mathrm{~N} l i$ [sic!]; Pl. N. NP $d a t$, MO $t$, Ket $t t p$, Tschl $t \ddot{a}$, tten, Tas B den (B auch det). Diese Endungen zeigen eine auffallende Übereinstimmung mit den Endungen der possessiven Deklination, und ihre gemeinsame Herkunft kann kaum bezweifelt werden. In den Indikativformen der 1., 2. und 3. Sg. unterscheidet sich im Selkupischen die Konjugation der intransitiven Zeitwörter von der der transitiven, z.B. (Mat 204 parku- 'schreien', vgl. par 'Geschrei'): 1. Sg. parkuang (N parkuak); 2. -kuand; 3. -kuan od. -kung; 1. Du. -ai; 2. -li, -ali; 3. -alge; 1. Pl. -t; 2. -lt, -alt; 3. -att (vgl. Gr.S. 529 parkuag). Die Endungen der intransitiven Verba zeigen eine auffallende Übereinstimmung mit den entsprechenden Endungen des Verbs 'sein': $\mathrm{N}$ eak, MO eang 'ich bin', 2. eand ( $n d<n+d$ ), 3. ean od. ek, $\mathrm{MO}$ eng (Mat 227-228). Obwohl wir kein klares Bild von der selkupischen Konjugation besitzen, scheint es auf Grund zahlreicher Angaben wahrscheinlich zu sein, dass die Indikativformen der 1., 2. und 3. Sg. der intransitiven Verba als ursprüngliche zweigliedrige Konstruktionen zu deuten sind: der Verbalnomenstamm wurde mit den entsprechenden. Formen des Verbums 'sein' verbunden, und diese Formen sind durch Einbusse ihrer Selbständigkeit zur Endung geworden.

Im Kamassischen sind die possessiven und die verbalen Personalsuffixe identisch und hängen eng mit den selkupischen Possessivsuffixen zusammen. Die Konjugation der intransitiven Zeitwörter unterscheidet sich nur in der 3. Person von der Konjugation der transitiven Verba. Die Sg.- und 
Pl.-Formen der intransitiven Verba sind Verbalnomina auf ${ }^{*} j \varepsilon$ (Sg. $i \varepsilon$, Pl. $j \varepsilon^{\prime}$ ). Im Dual der intransitiven Zeitwörter erscheint - genau wie in der Deklination -. das Zeichen des Duals $k$. (D-J 138, 146-159).

In den juraksamojedischen Texten sind die transitiven und intransitiven Zeitwörter nicht so deutlich unterschieden wie in den Paradigmen der Grammatik. Obwohl die Bearbeitung der komplizierten jurakischen Personalsuffixe eine besondere Studie erfordert, möchte ich mit einigen Bemerkungen doch demonstrieren, dass im Jurakischen die Personalsuffixe im wesentlichen genau so Possessivsuffixe pronominaler Herkunft sind wie im Selkupischen und im Kamassischen.

Bei den Zeitwörtern erscheinen in der 1.Sg. drei Personalsuffixe: $m^{\prime 1} ; \beta ; i, j$.

Das Possessivsuffix der 1 . Sg. $m$ ' ist in transitiven und intransitiven Zeitwörtern gleichermassen gebräuchlich: Arch tarem' jî̀'èm' 'so lebe ich' (430), BS sit $\beta \bar{a} \delta \bar{a} m$ ' 'dich zog ich auf' (286). - Das Personalsuffix $\beta$ ' erscheint sowohl in intransitiven als transitiven Zeitwörtern besonders in den Dialekten BS, MS, Kan und Arch, doch gibt es auch Belege aus dem Waldjurakischen: MS $j a \dot{a} \chi a \grave{n}^{\prime} \_$Dăe $\beta \beta \bar{\imath} \beta$ ' 'ich kam an einen

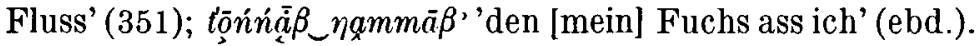
Das jurakische $\beta^{\prime}$ kann mit dem Possessivsuffix jur. 1.Pl. $\beta \beta a^{\prime}$, kam. $\beta a^{\prime}, b a^{\prime}, b \varepsilon^{\prime}$, wie auch mit dem $\beta$-Element der selkupischen und kamassischen I.Du., ferner mit dem $\beta$-Element des wogulischen Px. 1. Pl. $o \beta$ usw. zusammenhängen, dessen Zusammengehörigkeit mit dem Pronominalstamm ${ }^{*} m$. nach Liimola "äusserst unsicher" ist (Hist. Formenlehre d. Wog. 225-227) (anders P. Hajdú: ALH X, 105). Im Jurakischen

1 Nach Castrén ist das $d$-Element des Personalsuffixes $d m$ (vgl. waldjur. $t m, t$ ) "wahrscheinlich" ein euphonischer Laut (Gr. § 381). In Lehtisalos Texten finden sich zahlreiche Beispiele für Zeitwörter mit der Endung $\delta^{\boldsymbol{D}} m^{\prime}, \delta^{\hat{\partial}} m^{\prime}, \delta^{\circ} m^{\prime}, \delta^{u} m^{\prime}$ : OD $m \bar{a} \delta^{\nu} m^{\prime}$ 'ich sagte' (193), O ṕúu $\delta^{\hat{\partial}} m^{\prime}$ 'ich suche' (465), $\chi^{\bar{o} \delta^{o} m}$ ' 'ich fand' (164), tśū $\delta^{u} m$ 'ich ging hinein' (457) usw. In der Endung $\delta m^{\prime}$ ist also das Element $m$ ' ein Possessivsuffix, das Element $\delta(t)$ aber ein Ableitungssuffix, und so entsprechen die jurakischen Zeitwörter auf $\delta m^{3}(t m)$ den tawgysamojedischen Zeitwörtern auf $t u m$, tem, tim (Gr. S. 489), vgl. auch niletem niletm 'ich lebe'. 
ist das Px. 1. Sg. $m i^{\prime}$ aus der Dualkategorie in die SingularKategorie übergangen. Es findet sich in Castréns Texten auch in den Formen han-mi 'meinen Schlitten' (22, 23), hanemi (30) und han-ui (11, 12). Vgl. O jirílkkò $\beta$ 'mein Grossvater' (530), jirikikōmi id. (ebd.) Doch kommt das Possessivsuffix mi, min, vin bei Castrén auch in seiner ursprünglichen Dualbedeutung vor: "tie-vin gānan!» "führe meine Renntiere!» (10), b. teämin (22), c. tèmi (29) (vgl. selk. N Du $i, w i$, Akk. mi, wi usw., kam. $\beta^{2} i$ usw.) Das Personalsuffix $j$ der 1 . Sg. erscheint in der selkupischen possessiven Deklination und in der Konjugation als Personalsuffix, und in dieser Eigenschaft findet es sich auch in Castréns waldjurakischen Aufzeichnungen: tamdai $\sim$ tamdami ['bedecken'], tangai ['geben, bringen'], piingai ['sich fürchten'] (Mat 313, 315,316), vgl. lambai 'mein Schneeschuh' [? 1. Du.] (ebd. 308) usw. Bei Castrén heisst im Paradigma der possessiven Deklination die Nominativform der 1. Du. von sarmik 'Wolf' sarmikumi sarmikui (Gr. S. 246). In Castréns tundrajurakischen Texten fällt eine interessante Angabe auf, wo in der Verbform der 1. Du. die Endung $i \sim$

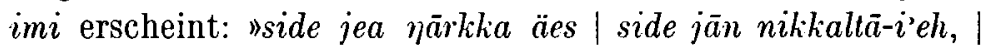
madaua äpta | tiet jān madangu-i» "wir, zwei Grosse des Landes seiend, | reissen ihn in zwei Teile | wenn wir ihn durchschneiden | schneiden wir in vier Stücke" (42), vgl. Variante b nikkalta-i-mi 'wir reissen', madangu-i-mi 'wir schneiden durch' (52). Joki verbindet das Dualzeichen $i$ mit dem die Pluralität bezeichnenden $i$-Element: das $i$ sei aus der Kategorie der Pluralität in die Kategorie der Dualität hinübergeraten (CSIFU I 225) und aus dieser dann - im Waldjurakischen in die Kategorie des Singulars. Den Entwicklungsgang verdeutlicht das obige Zitat: in beiden Zeitwörtern wurde das Px. der 1. Du. $m i$ abgeschliffen, seine Funktion hat in Variante a das die Dualität (Pluralität) bezeichnende Element $i$ übernommen. (Wir können auch beobachten, dass anstatt des auf das Dualobjekt hinweisenden Elementes $z a j u$ oft das auf das Pluralobjekt hinweisende Element ${ }^{*} j \varepsilon$ erscheint.) Infolge einer derartigen Entwicklung hat in den waldjurakischen Zeitwörtern auf $t m$ auch das Suffix $* t$. die Funktion des ${ }^{*} m$. vom Possessivsuffix der $1 . \mathrm{Sg}$. übernommen: $m$ wurde 
abgeschliffen, $t$ tritt als Personalsuffix in vielen Zeitwörtern

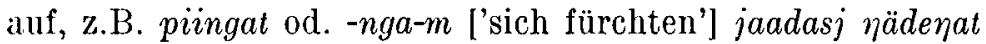
'ich ging zu Fuss' (Mat 316, 271); WNj měâ ${ }^{o} t t^{\prime} \bar{u} \eta \eta \bar{a} t$ 'in das Zelt trat ich ein' (407); nōp měât kō $\eta \eta \bar{a} t$ 'ein Zelt traf ich' (ebd.).

In der possessiven Deklination tritt das Px. der 1. Pl. $\beta \beta p^{\prime}$ ebenso auf wie in den transitiven und intransitiven Zeitwörtern: OD síd'e $\chi \bar{a} l m e r a \beta \beta p$ ' 'unsere zwei Toten' (188);

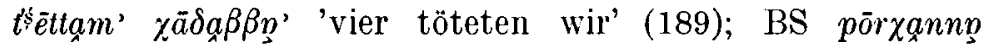

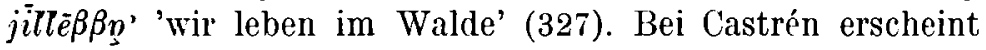
in den Nominativformen der Paradigmen der possessiven Deklination als Px. der 1. Pl. teils $m a^{2}$, teils $w a^{\prime}$ bzw ${ }^{*} n a^{\prime}$, im Waldjurakischen $m a^{\prime}, w a^{\prime}$, $n a^{\prime}$ (Mat 308-310). Vgl. BS

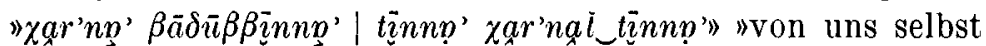
aufgezogen [sind sie], | die Renntiere sind unsere eigenen

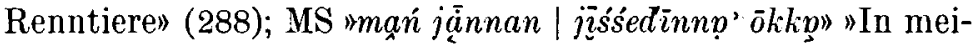
nem Lande | [sind] viele Unvernünftige» (259), vgl. MB (BS)

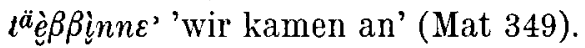

Das Px. der Pl. mit dem n-Element hängt zweifellos mit dem Personalsuffix $n$ der 2 . Sg. zusammen, das nicht nur in intransitiven, sondern auch in transitiven Zeitwörtern sehr häufig rorkommt: (C) niedam gongun 'du findest den Weg' (105), vgl. niedam gōngur id. (72). Bei Castrén liegt das Personalsuffix $n$ auch in der vollständigeren Form na vor: "siu jāle mindāna" "sieben Tage gehst du" (35), in den Varianten $\mathrm{b}$ und $\mathrm{c}$ mindān $(49,56)$. Die vollständigere Form findet sich auch bei Lehtisalo vor dem verstärkenden Element mp: PD

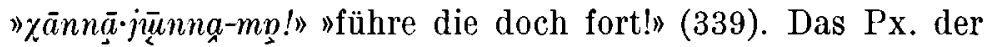
1. Du. $n i \sim n i$ ' wird an ein Nomen, an transitive und intransitive Zeitwörter gleichermassen angehängt: MS jẳin $i^{\prime} \dot{m} \bar{u} s^{\prime}$

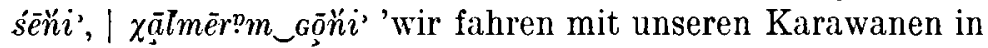
unser Land, | fanden einen Verstorbenen' (355). Die Possessivsuffixe mit einem $n$-Element haben in den obugrischen Sprachen ihre Entsprechungen von deutlicher pronominaler Herkunft (M. Liimola a.a.0. 214, 222-225, 227-232).

Das jurakische, die 2. Person bezeichnende Possessivsuffix mit dem Element $r$ kann dem selkupischen Possessivsuffix mit dem Element $l$ und dem kamassischen mit dem Element 
$\boldsymbol{a}$ gleichgesetzt werden. Vereinzelt kommt in den Zitaten ein Px. der 2. Sg. $l$ auch im Jurakischen vor: O "paxdàr ṕéntśel"

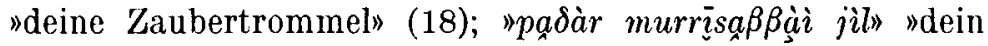

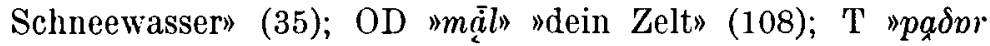
$\chi \overline{a j j e \bar{e}}$ " "deine Sonne" (95). Auch in Castréns Paradigmen für die possessive Deklination kommt das die 2. Person bezeichnende Possessivsuffix mit dem l-Element vor: Sg. jaml, Du. jamli, Pl. jamla (jam 'Meer' Gr. S. 256), vgl. 'Mensch' (257), 'Brief' (259), 'Zelt' (261). Aus Castréns waldjurakischen Aufzeichnungen können wir mehrere Angaben anführen: munapśel [dein Bart], -śeli, -śela (Nat 310) usw. Das Px. der 2. Sg. kommt in Castréns Texten auch in der Form ra vor: jädarmāra jādarmar 'dein Besuch' (218), tumdtsaekīira 'du kennst [es] wohl' (77).

In Castréns und Lehtisalos Texten kommt das Px. der 2.Sgr. *t. auch mit einem vokalischen Element vor: C »nisjem-da hädāua'ah, | njevemda hädäua'ah" "deinen Vater erschlugen wir, | deine Mutter erschlugen wir" (272); T "jūinnamì tumià $\beta$ " "ich kannte dein Pferd" (94); O "padàr $\beta \bar{a} \eta k a \chi a \bar{\delta} \delta a n i^{p} p \bar{n} n$ ' tarppāon'!" "tritt aus deiner Höhle heraus!" (42); (C) »hartta nisenad hādaieda" "deine eigenen Väter tötetest du" (228); MS "ńä $\beta i$ ńêr $t \bar{a} \delta^{p}$ !" "gib das andere Weib!» (262). Das Px. der 2. Pl. * $t a$ ' erscheint sowohl bei Nomina als auch bei transitiven und intransitiven Zeitwörtern. In transitiven Zeitwörtern haben * $t a^{\prime}$ und $r a^{\prime}$ die gleiche Funktion: (C) "mōnsanguu ma$d \bar{a} d a, \mid t j i$ ni $(m)$ idāra $(\sim i d \bar{a} d a) ! »$ "meinen Schenkel schneidet

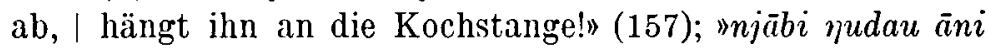
madāra $(\sim \operatorname{mad} \bar{a} d a) \mid \overline{a n i}$ nidāra!" "meine eine Hand | schniedet wieder ab, | hängt sie wieder auf!» (159).

Das Px. der 3.Sg. ${ }^{*} t a,{ }^{*} t \varepsilon$ erscheint im Waldjurakischen als ttaj, taj. Vereinzelte Angaben liegen auch aus dem Tundra-

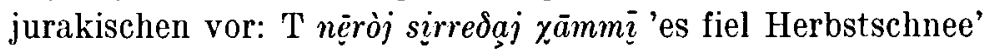

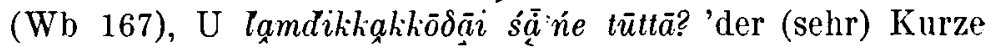
kommt wann?' (581). In Castréns Texten entsprechen dem Possessivsuffix ${ }^{*} t a j,{ }^{*} t \varepsilon j$ die Formen dah, deh (djeh): njanaej tirädah 'seine silberne Faust' (77), niedeh 'seine Frau' (83), atseki nimdjeh 'der Name des Knaben' (63), vgl. die Varianten des Px. der 3. Sg.: (C) die, (L) de, t'e usw. Für die Form dan 
des Px. der 3. Sg. finden sich vereinzelte Belege nur in Castréns Texten: sājuu sjurvuida'ah | narodan minjes 'die Räuber begannen zu laufen | ihre Speere tragend' (272); sjurnje jëndat(ah) | anodan seijuueh 'im drehenden Strom | ging ihr Boot unter' (297). - Bei Castrén erscheint das Px. der 3. Pl. meistens als $d o, d u$, oft als $d o^{\prime}, d u^{\prime}$, manchmal als $d \bar{o}(3), d o h(180)$, aber auch als $d j o, d j u(156)$, bei Lehtisalo als $\delta o^{\prime}, \delta u^{\prime}$. Wir besitzen mehrere Angaben für die Form don, dun, tun des Possessivsuffixes in Castréns Texten: äeuwadon gadipi 'sie kratzen sich den Kopf' (161); tuton ( tutun) tumah 'das [ihr] Feuer begann zu brennen' (123). Vereinzelte Angaben lassen sich auch aus Lehtisalos Texten zitieren: MS jēr $\beta^{\circ} t t a \bar{\delta} \bar{n} n_{-}$ Dqñne $\beta \beta \bar{e}$ 'ihr Fürst ist vorhanden' (260); MS "pēllemdōn $D \bar{a} \eta-$

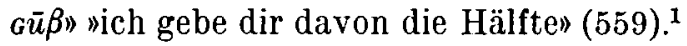

In den Paradigmen der Grammatik sind die Akkusativ + Px. 1. Sg.-Formen mit dem Nominativ + Px. 1. Sg. identisch, deren Endung teils $m$, teils $u(\beta)$ ist (Gr. $\S 412$ ). Aus den Texten können wir Angaben anführen, bei denen die Akkusativendung mit der 1. Sg.-Endung des prädikativen Verbs identisch ist: Arch $m \bar{u} \delta a m^{\prime} \eta \ddot{a} e \grave{e} \delta \bar{a} \delta^{a} m^{\prime}$ 'die Karawane [= meine Kara-

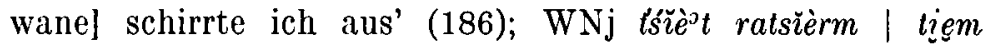

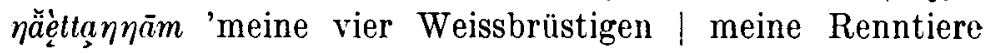
schirrte ich aus' (410); - (C) hāptuu njamau 'meinen Renntierochsen fing ich ein' (302); Arch nüè $\beta \beta \partial \grave{o} \beta$ jillà $\beta$ 'ich hob meinen Kopf' (423). Es erhebt sich die Frage: was ist das Element $m^{\prime}$, bzw. $\beta^{\prime}$ in den nominalen Formen, eine Akkusativendung oder ein Px. der 1. Sg.? Nach Lehtisalos feiner Transkription sind die beiden Elemente der Form nach identisch: Akk. $m^{\prime}=$ Px. der 1. Sg. $m^{\prime}$. In beiden Endungen weist der Kehlkopfverschluss auf einen geschwundenen Konsonanten hin (der dem vokalischen Element gefolgt war). Aufschlussreich ist die folgende Angabe: (C) "hüve sit tābada $\mid$ tjukī dem

${ }^{1}$ Das Dualzeichen $h \bar{a}$ in Castrèns waldjurakischen Aufzeichnungen weist auf eine Verbindung *ka $+j \varepsilon$ hin. Das Element *kai lāsst sich mit dem tawgysamojedischen Dualzeichen kai $\sim$ gai identifizieren, wo $i$ das Zeichen der Pluralität ist. In einer Angabe erscheint bei Castrén $n$ als drittes Element: poonga 'Netz': poomga-ha $\bar{a}(n)$ (Mat 301). Vgl. noch Gr. S. 225. 
njamas?" "Wer befahl dir | dieses Renntier zu nehmen?" (36). Die Form dem ist nicht eindeutig; in Variante $b$ haben wir tim (50), in Variante c temi (56), wo aber die Endung $m i$ identisch ist mit dem Px. der 1. Sg. mi.

Der häufige determinierende Gebrauch der Possessivpronomina in den uralischen Sprachen ist allgemein bekannt (für das Jurakische vgl. ALH III, 308-314). In einer aus dem PD-Dialekt aufgezeichneten Erzählung (120-132) drückt das an die Subjekte und die mit Kasusendungen versehenen aldverbialen Bestimmungen tretende $\mathrm{Px}$. der 2. Sg. $r$ die Beziehung des Erzählers zum Zuhörer und die des Zuhörers zu den ihm erzählten Geschehnissen aus. In der Erzählung finden sich solche stehenden Wendungen wie nădèdananna zäsa $\beta \beta \bar{a} r$ 'der [dein] mit Treibnetz fischende Mann', śide jåderttp lütsẹer 'die [deine] zwei russischen Schmiede' (es sind wichtige Personen in der Erzählung). - Es gibt auch solche Sätze, in denen das persönliche Verhältnis des Erzählers zu den Geschehnissen durch ein an irgendein Satzglied angehäng-

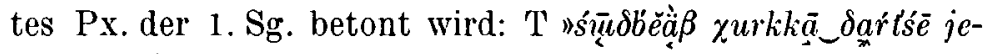

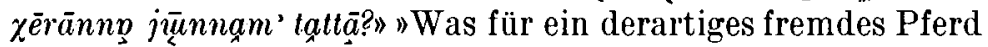
bringt der Riese?» [mein Riese, d.h. von dem ich spreche]

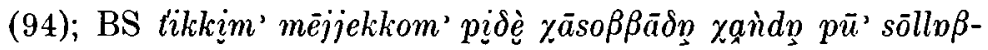
$\beta \bar{e} \delta \underline{y}$ 'Diese Schwiegertochter [meine diese, meine Schwiegertochter] band ihr Mann hinter seinen Schlitten' (545); OD

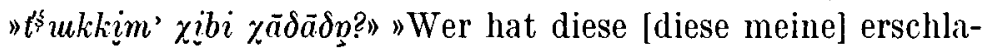
gen?" (108).

Vom juraksamojedischen Gesichtspunkt aus scheint die Konzeption natürlich zu sein, dass der Sprecher, um das Objekt mit Nachdruck zu bezeichnen, das enklitische Personalsuffix der 1 . Person Sing. ${ }^{*} m$. bzw. das aus diesem stammende Possessivsuffix zur Grundform hinzufügte, woraus dann ein determinierendes Element, später eine unpersönliche verstärkende Partikel entstanden ist. Dieses verstärkende Element dürfte dann in einigen uralischen Sprachen zum Zeichen des Akkusativs geworden sein (vgl. im Kamassischen die beim unbezeichneten Objekt auftretende verstärkende Partikel mit einem $\beta$-Element und die Akkusativformen in Castréns jur. Paradigmen mit $u$ ). Im Jurakischen werden die verstärkenden Partikeln $m$. und $\beta$. häufig gebraucht, und die 
aus Castréns Grammatik (S. $130-440$ ) bekannte sog. fragende Konjugation ist auch nichts anderes als dic mit $m$. bzw. $\beta$. versehenen Formen der Zeitwörter.

Die verbalen Personalsuffixe sind alle - mit Ausnahme des Elementes $j, i$ - Possessivsuffixe pronominaler Herkunft ${ }^{\mathbf{1}}$, und sie werden alle (im Waldjurakischen auch das Element $j$ ) ebenfalls in der possessiven Deklination gebraucht. Die Angaben aus den jurakischen Texten bezengen, dass der Gebrauch des bezeichneten und des unbezeichneten Objekts nicht von der sog. subjektiven oder objektiven Konjugation des Verbs abhängt, und auch die Form des prädikativen Verbs hängt nicht von der Form des Objektes ab. In jurakischen Texten lässt sich eine gewisse Differenzierung in der Konjugation der intransitiven und der transitiven Zeitwörter bemerken, aber im wesentlichen liegt im Jurakischen derselbe Zustand vor wie im Kamassischen: die intransitiven und die transitiven Verben unterscheiden sich nur in den Formen der 3. Person voneinander. Die objektive Konjugation im Indikativ ist nichts anderes als die possessive Deklination des Verbalnomens auf $* j \varepsilon$ : der Verbalnomenstamm hat die Bedeutung eines Nomen actionis. Die Konjugation der transitiven und intransitiven Verben war ursprünglich dieselbe. Mit jurakischen sprachlichen Tatsachen lässt sich die Differenzierung wie folgt nachweisen: 'kommen' *tol( $e)+j \varepsilon$ Sg. 1. $t \bar{o} m$ ' 'Kommen $(-\mathrm{ich}=$ ) -mein'; 2. tōn 'Kommen (-du $=$ ) -dein', 3. $t \bar{o}$ 'Kommen'; 3. Pl. $t \bar{o}$ ' ('= ?); -- 'verlassen': *kājje $+j \varepsilon$ 1. Sg. $\chi \bar{a} \bar{j} j \bar{e} m$ ' $(\sim \chi \bar{a} j \bar{j} \bar{e} \beta)$ 'Verlassen $(-\mathrm{ich}=)$-mein'; 2. $\chi \bar{a} j \bar{j} \bar{e} n$ $(\sim \chi a j j \bar{e} r)$ 'Verlassen $(-\mathrm{du}=)$-dein', 3. $\chi \bar{a} j \bar{j} \bar{e}$ 'Verlassen' vgl. $\chi \bar{a} j \bar{j} \bar{e}-\delta n$ 'sein Verlassen'; 3. Pl. $\chi a j j \bar{e}-\delta u$ ' 'ihr Verlassen'.

Die possessive Dellination der intransitiven Verbalnomina auf $* j \varepsilon$

(Die sog. "reflexive Konjugation")

Nach Castrén haben die Verba mit reflexiver Bedeutung in den nordsamojedischen Sprachen eine eigenartige Konju-

${ }^{1}$ Die Herkunft der Possessivsuffixe mit einem Element $l \sim r$ ist nicht geklärt. 
gation. Die reflexive Bedeutung wird zusammen mit dem Charakter $j, i i, i$ durch eigene Personalsuffixe angegeben (Gr. $\$ 357,373,377,379,490$ ). In den Paradigmen kommen nur transitive Zeitwörter vor, aus den Texten hat man aber nur Formen mit intransitiver Bedeutung. Diese zeugen davon, dass die sog. reflexive Konjugation nichts anderes ist als die possessive Deklination des mit dem Suffix ${ }^{*} j \varepsilon$ gebildeten Verbalnomens intransitiver Bedeutung. In den Texten kommt das Verb mit der Bedeutung 'ankommen' oft vor. Dem aus diesem Verb gebildeten Verbalnomen werden Possessivsuffixe angehängt: BS mań těęêj-ji- $\beta^{\prime}$ 'ich kam an' (332); mań

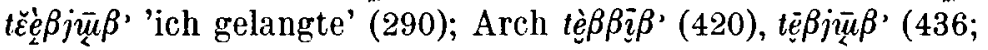
$i>u$ vor dem Px. 1. Sg. $\left.\beta^{\prime}\right) ; 0$ mnaxà' tè $\beta \beta \overline{e n n " ~ " w e i t h e r ~ b i s t ~}$ du gekommen" (441); W Nj »kuñéăt täésjen?" "Woher kommst

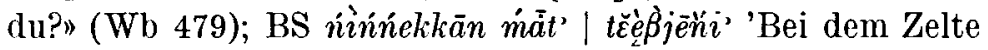
meines älteren Bruders | kamen wir [Du.] an' (334); MS $\chi \grave{o ̀ i-~}$ $\delta \bar{a} n$ tăę $\beta \beta \bar{\imath} n n \underline{\text { Q }}$ ' 'Wir kamen nach Koida' (554). [Für die 2. Du. und 2. Pl. von diesem Verbalnomen haben wir keine Angaben; vgl. O "pin' $\eta \bar{a} m t \bar{z} d i$ '» "ihr setzt euch [Du.] . . hinaus" (10); W Ni "tśū $\beta$ jurokjetty'!» "stehet auf!» (141).] In Lehtisalos tundrajurakischen Texten hat die Form der 3 . Sg. einen Kehlkopfverschluss am Ende: $O t \bar{a} \delta \underline{\underline{x}} t \grave{e} \beta \beta \overline{\underline{t}}$ ' 'dann kam er an' (444). Formen dieses Typs lassen sich aus den Mundarten T PD BS MS Kan Arch in bedeutender Anzahl anführen. In den waldjurakischen Angaben erscheint zum Teil ein Kehlkopfverschluss, zum Teil ein $\mathrm{Px}$. der 3. Sg.: $\mathrm{Nj}$ tăęęjettp 'er kam

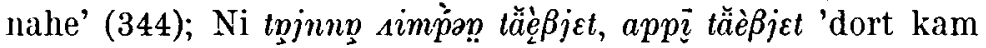
der Adler ein, dorthin kam der Bär' (138). Das Prädikat auf $j \varepsilon t$ spielt eine bedeutende Rolle im Märchen Nr. 50 aus dem Ni-Dialekt (132-147). Ebenda erscheinen die Prädikate in der 3. Pl. mit der Endung $j \varepsilon t$ ': $t \bar{o} j$ 'jēn tăèe $\beta j \varepsilon t$ ' 'sie kamen zu dem grossen See' (138, vgl. 84, 137, 142). Die entsprechende Form hat im Tundrajurakischen die Endungen $\delta^{p}$, und $\delta^{\prime}$ : 0

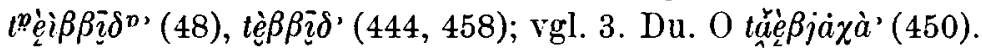
Es gibt zahlreiche Angaben aus den Texten für Verbalnomina mit der Bedeutung 'sich setzen, sitzen', 'sich schlafen legen, einschlafen, schlafen' usw. Dass es sich bei allen diesen Formen nicht im geringsten um Reflexivität handelt, beweisen 
die mit Possessivsuffixen versehenen Verbalnomenformen der Zeitwörter mit der Bedeutung 'leben' und 'sterben':

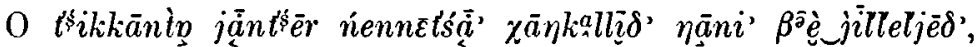

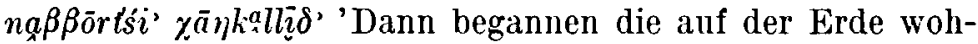
nenden Menschen zu sterben, fingen an in Armut zu leben, begannen ohne Essen zu sterben' (16, vgl. 9. 46, PD 64). Die inchoative Bedeutung wird durch das Suffix $\eta k a+l$, bzw. $l$ angegeben, vgl. (C) jileliiedo 'sie begannen zu leben' (17), jililjed' id. (33).

An das nominale Prädikat werden die folgenden Possessivsuffixe angehängt:

1. Sg. $\beta^{\prime}\left(? m^{\prime}\right) ; 2 . n ; 3 .{ }^{\prime},{ }^{*} t,{ }^{*} t a ;$ Du. $n i{ }^{\prime}\left(n e^{\prime}\right) ; t i^{\prime} ;\left(j \dot{a}-\gamma \dot{a}^{\prime}\right) ; 1$. Pl. ${ }^{*} n a^{\prime}\left(? \beta a^{\prime}\right) ; 2 .{ }^{*} t a^{\prime} ; 3 .{ }^{*} t,{ }^{*} t a^{\prime}, t o^{\prime}$.

\section{Der Konjunlitiv}

Der Charakter des jurakischen Konjunktivs nach Castrén ist $j i, i, n i$ (Gr. § 469). (Es soll gleich bemerkt werden, dass das Element $n i$ keine Formvariante des Elementes $j i$ ist.) Die Konjunktivformen erscheinen in Zitaten, in der Rede der Personen, in Fragen, Antworten, Verneinungen, in Wünschen mit imperativischer Betonung, deren Erfüllung eventuell nur in der Zukunft erfolgt. Der Konjunktiv drückt kein Zeitverhältnis aus. Die formale Gleichheit einzelner Formen des Konjunktivs mit den entsprechenden Formen des oben behandelten nominal gearteten intransitiven Verbalnomens ist so auffallend, dass wir die identische Herkunft der beiden Verbalnomenstämme kaum in Zweifel ziehen können, z.B.

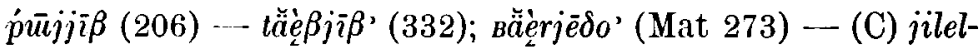
jiedo (17). Die lange Quantität des Vokals in dem Suffix dürfte durch die Reduplikation des Elementes *je verursacht worden sein. (Für solche Reduplikation zum Ausdruck des nachdrücklichen Wunsches kennen wir auch einige andere Beispiele.) Die Absonderung der subjektiv und objektiv konjugierten Kategorien wird durch die Angaben aus den Texten nicht bestätigt: WLj "mań kuptp̧â miñdêât ${ }^{N_{\eta}}$ "

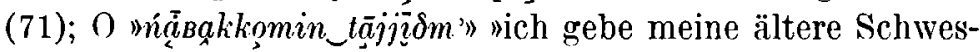




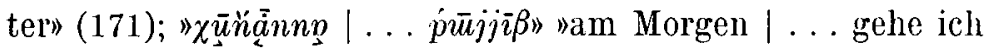
ihn suchen» (206). - Wir haben viele Angaben für die Form der 2. Sg.: (C) "hūnjāna müusijin!" "morgen fahre mit deiner

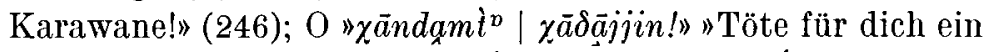

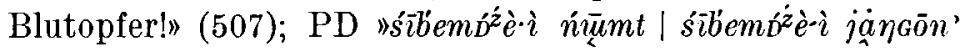

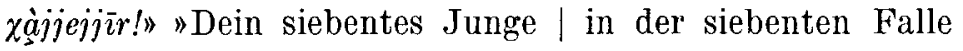
lasse zurück!» (540, Zaubergesang; vgl. 108, O 163); "pū $\overline{\bar{e}}$,

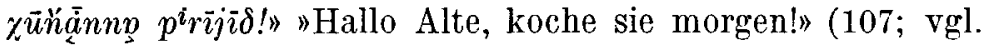

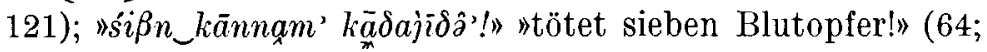
vgl. MS 307); (C) "tjuki $j \bar{a} d$ punjān beä-ji-ua» "Von dieser Stelle beginnen wir zurück [zu gehen]" (246). Die Form der 3. Sg. der intransitiven Verba endet bei Castrén auf $j e h$, bei Lehtisalo auf jje: (C) »manj jan jied tōjeh» "er möge auf mein Land

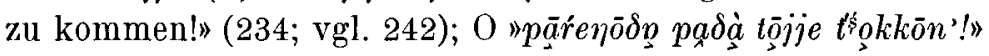
"Der Kaiser, er mag hierher kommen!" (41, vgl. 165). Auch das unpersönliche Zeitwort mit der Bedeutung 'es gibt nicht' hat eine Konjunktivform: (C) "jierengōda jängōjeh»»Wächter mögen

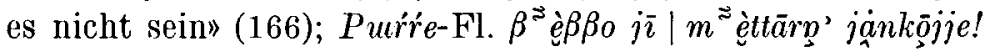
'Besitzer | schlechter Vernunft | seien nicht!' (495; Zaubergesang). - In transitiven Verben erscheint das Px. der 3. Sg.: (C) wä̈sako manjijēda ( manjijieda) 'der Alte betrachtet

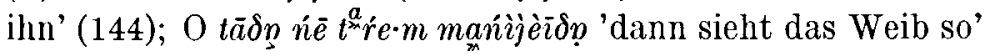
(446); (C) "uen amjedoh!» "mögen die Hunde [sie] fressen!» (238); U jeśśenca'nv ōstárèm 'Bäęerjēoo' 'Auch mit Eisen fängt man

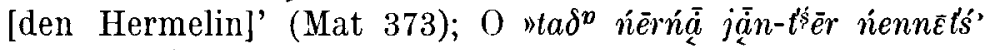

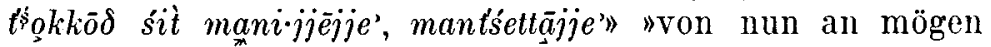
dich die auf der Erde wohnenden Menschen hier sehen, mögen sagen" (9). - Für die Formen der 3. Du. gibt es keinen ein-

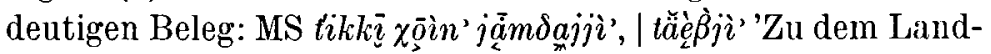
rücken begaben sie sich mit ihrem Zelt, | kamen an' (277, 348);

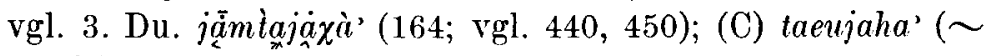
taeujā) (144).

Das Verbalnomen passiver Bedeutung auf $* \beta . b z w . * j .+\beta$.

"Ein eigentliches Passivum besitzt das Samojedische nicht", bemerkte Castrén (Gr. § 479). Lehtisalo erblickte die Funktion 
der Ableitungssuffixe ${ }^{*} u,{ }^{*} \ddot{u}$, bzw. ${ }^{*} j+u, \ddot{u}$ darin, dass sie aus transitiven Zeitwörtern intransitive bilden (AblSuff 40 42). Die ursamojedische Form des Suffixes war $* \beta$. bzw. $* j .+\beta$. (vgl. Erkki Itkonen, Scandinavica et Fenno-Ugrica 183-191). Seiner ursamojedischen Funktion gemäss bezeichnete das Suffix den Zustand; von dieser Funktion kann die passive Bedeutung abgeleitet werden. (Das Suffix $j$ könnte auch an sich passive Bedeutung haben z.B. im Verbalnomen $a d i-<* a d+j \varepsilon$ 'sichtbar sein'). Die mit dem Suffix $* \beta$. bzw. $* j$. $+\beta$. gebildeten Verbalnomina erscheinen mit den Endungen $\bar{o}, \bar{u}(\bar{u})$ im Jurakischen. Wenn das Element ${ }^{*} j$. an einen konsonantischen Stamm trat, führte es die Mouillierung des Konsonanten herbei.

Die aus intransitiven und transitiven Verben stammenden passiven Verbalnomina kommen gleichermassen besonders in Zitaten vor, sie werden auch mit einigen Possessivsuffixen verbunden. Sie haben keine Formen, die als Verba finita angesprochen werden können.

Ein solches Verbalnomen ist z.B. das Wort mit der Be-

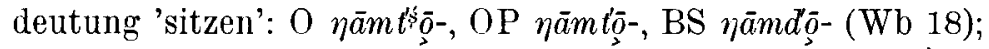

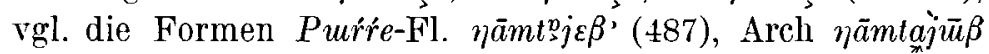
(437), O yamti $\beta$ ' (19) usw. ['mein Sitzen' usw.]. In Castréns waldjurakischen Aufzeichnungen finden sich Formen wie:

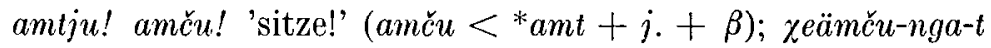
'ich sass' (Mat 266). ${ }^{1}$ Weitere Beispiele: WNj pi' $\eta \bar{a} m t^{\prime}{ }^{\prime} u$ $-\eta \eta \bar{a} \cdot t, \mid j \bar{a} r \dot{r} a \eta^{\prime} \eta \bar{a} m t s u \eta \eta \bar{a} \cdot t$ 'in der Nacht bin ich ("sitze ich"), am Tage bin ich ("sitze ich")' (415); MS tarèm' jā $\delta \bar{a} m^{\prime}$ | mōnn $n^{a}$ settī̧' | àmóōsettim. 'So schreite ich, | falle immer

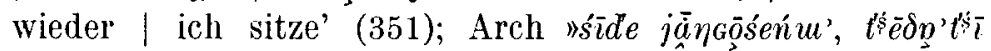

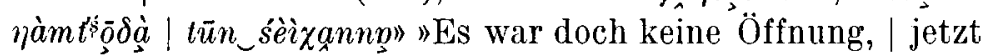
da sitzt er | im Herzen des Feuers" (181). - Dem Verbalno-

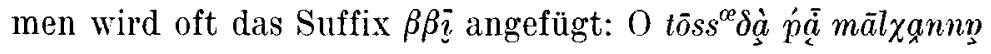

1 Die Affrikate in diesem Wort ist ein sekundärer Laut, wie in den Wörtern nači, eetsi usw. 'ist sichtbar' (vgl. nat-je-a id. Mat 267). Vgl. noch selk. Jel ačang $\left(<^{*} a t+j+a n g\right)$ 'ich bin sichtbar' (Mat 10). Die

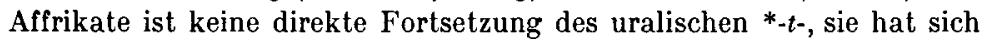
aus der Verbindung $*_{t}+j$ entwickelt. Es scheint wahrscheinlich, dass der uralische Laut *-t- auch in anderen Fällen keine samojedische Affrikaten-Fortsetzung hat (vgl. Beitr. 229-230 Anm.; CompGr. §111). 


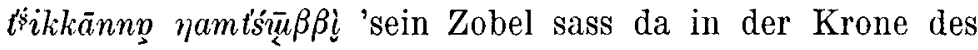
Baumes' (47; vgl. 19, 40, 441, 450, MS 296). Mit dem Passivstamm wird das verbalnomenbildende Suffix ${ }^{*} t a+j \varepsilon$ verknüpft, dessen Lativ (+ Px. 3. Sg.) in der folgenden Angabe

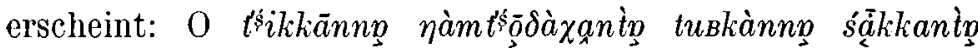
sappā $\delta u^{\prime}$ 'Während er da sitzt, schlugen sie mit der Axt in sein Gesicht' (113, vgl. 19).

Es finden sich sehr viele Belege in den Texten für das mit dem Suffix $* \beta$ gebildete Verbalnomen 'halt machen und ein Zelt oder Zelte errichten [= lagern]'. Stammvarianten: O

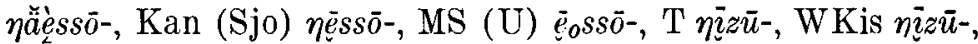
vgl. Kan $\bar{e}_{o} s s a-($ Wb 26). Auch in Castréns Texten finden sich Formen mit a-Stamm: "tjiken üäsa-dōn!" "dort schlage die Lager auf!” (247); niernjäna| mead neäsawioi $(\sim$ isavioih $\sim$ üäsavioih) 'In der Richtung vor ihm | hatten die Zelte ihr Lager aufgeschlagen' (122). In Castréns Angaben weist der Vokalismus der ersten Silbe eine grosse Mannigfaltigkeit auf: in den Stämmen auf $\bar{o}, \bar{u}$ sehen wir $e \ddot{a}, \ddot{u} \ddot{a}, \vec{\imath}$, in den Formen der 3 . Sg. der mit ${ }^{*} j \varepsilon$ gebildeten Stämme aber $\ddot{u} \ddot{o}(4), i_{o} \ddot{o}(131$, 265), $\ddot{u} \ddot{a}(12,118,147,171,212,217), i_{o} \ddot{a}(131,145,146$, $171,217), \bar{e}, \bar{l}(26,118)$. Bei Lehtisalo sehen wir die Vokale $\bar{e}_{0}, \bar{e}, \vec{\imath}$, bzw. $\ddot{a} e,(\mathrm{OP} \ddot{a} e i)$. Es ist wahrscheinlich, dass der urjurakische primäre Verbalstamm * ${ }^{*}$ oise $\sim * \beta$ oese oder ${ }^{*} \beta$ oisa

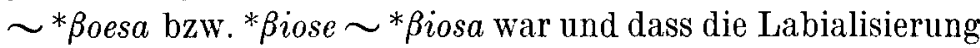
des Diphthongs in Castréns Angaben durch den ursprünglichen Anlaut ${ }^{*} \beta$ - verursacht wurde. Der Schwund von * $\beta$ bzw. seine Substituierung durch einen sekundären Nasal gab Anlass zur Entwicklung nach verschiedenen Richtungen, wobei auch der Vokal der zweiten Silbe eine Rolle spielte. Mit den $\bar{o}-, \bar{u}$-Stämmen können Suffixe mit passiver Bedeutung sowie - in Zitaten - solche, die Irrealität bezeichnen, ver-

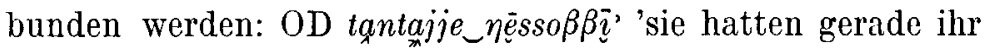
Lager aufgeschlagen' (379), vgl. (C) janga jahana | njahar

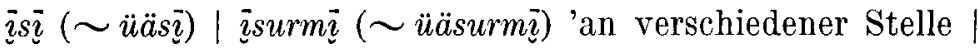
drei Zeltlager hatten sich gelagert' (118). Bei dem Verbalnomen auf ${ }^{*} t a+j$. steht der Kehlkopfverschluss am Wortende anstatt des geschwundenen Possessivsuffixes: MS sì $d^{\prime} e$ měă',

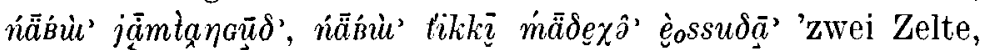


die einen begeben sich auf die Umzugsreise, die anderen lassen sich auf diese Zeltstätten zum Wohnen nieder' (594).

Wir besitzen zahlreiche Angaben für das zur Wortfamilie ron ung. hagy gehörende tundrajurakische Verbalnomen $\chi \bar{a} j \bar{j} \bar{o}$, waldjur. kājjō 'zurückbleiben' (Wb 164), das vom Stamm $\chi \bar{a} j j \grave{j}$ - 'zurücklassen' stammt und eigentlich die Bedeutung 'zurückgelassen, verlassen werden' hat. Das Verb 'zurücklassen' ist ein * $a$-Stamm (vgl. Ip. guodde-) und es existiert auch im Jurakischen als ein $a$-Stamm: (C) paridienje njüdeäm | paertse häja-vi $i_{0} i h$ 'den Jüngsten des Schwarzen liess sie zum Kämpfen da' (200). Das passive Verbalnomen erscheint sowohl bei Castrén als auch bei Lehtisalo in prädikativer Funktion besonders in einer mit dem Suffix $\beta \beta \vec{\imath}$ gebildeten Form: $\chi \grave{a} j \bar{j} \bar{u} \beta \beta \bar{\imath}(108,117,255,287,318$ usw.), vgl. Arch $\chi \bar{a} j \bar{j} \bar{u} \beta \beta e$ (172). In einem Zaubergesang finden wir es in einer interessanten Verbindung mit dem Verb 'sein' als Form der 1. Sg.:

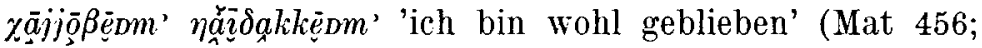
die Form mit dem Stamm $\chi \bar{a} \bar{j} j \bar{o}$ drückt die Passivität, die das Suffix *ta und die Partikel $k k \bar{e}$ enthaltende Form des Verbs 'sein' drückt - da es sich um eine Vision des Zauberers handelt - Irrealität aus). Als Prädikat für die Form der 3. Sg. kommt auch das mit dem Suffix *ma $+j$. bzw. * $\eta a$ gebildete Verbalnomen vor: WNi kājjummī (82), kāäjummą̆̋ j (ebd.),

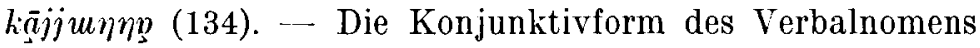

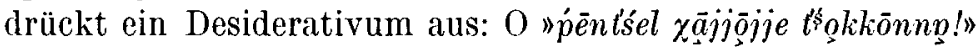
"Deine Zaubertrommel möge hier bleiben!" (19); Kan "mía'jen-

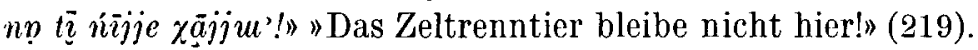
Das aus dem Passivstamm mit dem Suffix *ta $+j \varepsilon$ gebildete Verbalnomen kommt - nach vereinzelten Angaben - auch in prädikativer Funktion vor: (C) mmanj häjodādm ( häjo-

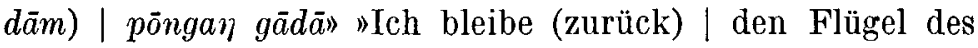

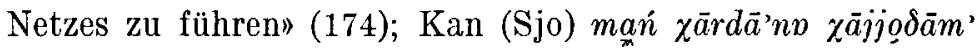
sè $\eta \gamma a s_{x}$ 'ich bleibe für die Nacht im Dorf' (Wb 164); "tiki jāna| häjodāda ( hājohdāda)» "An dieser Stelle | bleibt ihr»

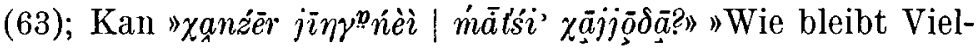
frass [Männername] ohne Zelt?" (216). - Den nominalen Charakter der Verbalnomina auf $\bar{o}, \vec{u}$ veranschaulicht die folgende Angabe, wo $\chi \vec{a} j \bar{j} \bar{o}$ - in der Funktion des Nomen possessi 


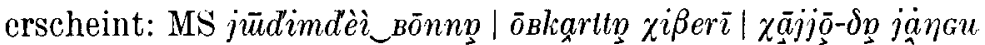
'Im zehnten Jahre | keinen einzigen übriggebliebenen | Menschen gibt es' (274).

\section{Verbalnomina auf ${ }^{*} m a,{ }^{*} m \varepsilon$}

Das mit dem Suffix *magebildete Verb a $1 \mathrm{n}$ o m e $\mathrm{n}$ bezeichnet im prädikativen Gebrauch einen Zustand und hat passiven Sinn: OD śinnūop sümmp 'Der Ne-

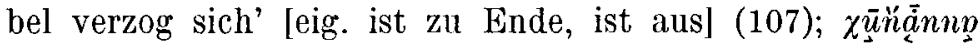

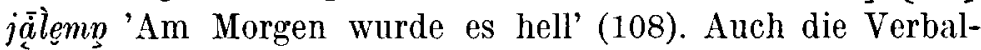
nomina auf $\bar{o}, \bar{u}$ haben Formen, die mit dem Suffix *ma ge-

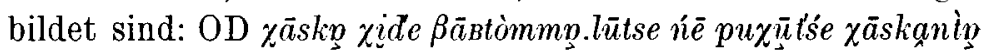

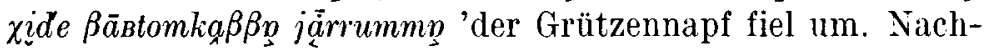
dem der Grützennapf umgefallen war, begann das russische Weib zu weinen' [eig. war' im Weinen] (72; vgl. 108, Kan 234);

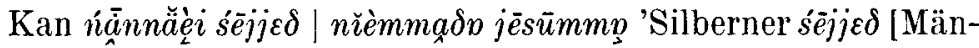
nername] erwachte aus dem Schlaf' (232). Sehr selten wird dem Verbalnomen ein Possessivsuffix angehängt: O "padsàri $m \bar{a} l l \bar{e} \beta^{z} \grave{e} s s a \cdot i m m a{ }^{\prime}{ }^{\prime} "$ "Ihr [Du.] seid schon alt geworden" (10).

Das zweite Element des zusammengesetzten Suffixes *ma $j$. ist mit dem passivischen verbalnomenbildenden Suffix ${ }^{*} j \varepsilon$ identisch. In dieser Form ist das Suffix *maj nur im Waldjurakischen vorhanden. Ein Beleg findet sich aber auch in Castréns Texten: jālumdata jāngumah 'das Tageslicht verschwand' (227). Für das Verbalnomen auf maj können wir aus dem Ni-Dialekt viele Angaben anführen, besonders aus dem

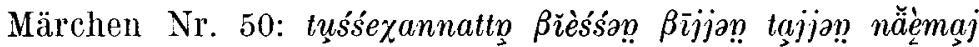
'In seiner Tasche war Eisendraht' (133; vgl. 132, 134, Nj

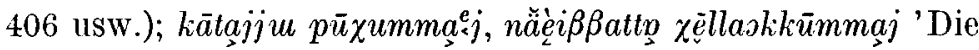
Grossmutter ist alt geworden, ihr Kopf ist grau geworden'

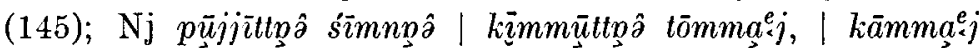
'Aus seinen Nasenlöchern | ist Blut gekommen, | er ist gestorben' $(417$; vgl. 414, 416). Für Verbalnomina, die von transitiven Verben stammen, gibt es wenig Belege: Ni täjnnn Áim-

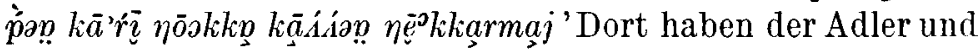
der Vogel $k \bar{a} \cdot r i$ viel Fische gezogen' (138); $\mathrm{Nj}$ "țtū jōrokkar- 
maj'" "Ihre Renntiere sonderte sie aus" (412); tinnśśạn kōrmąa $j$ '[er] prüfte den Lasso' (416).

In den wen igen Verbalnom i n a i u f mīlässt sich die Endung wahrscheinlich auf die Endung maj zurück-

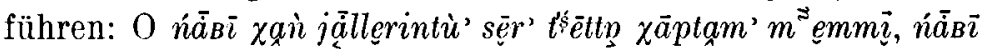

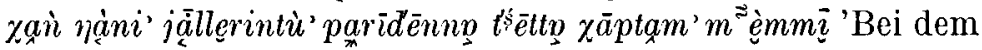
einen Schlitten waren vier ganz helle Renntierochsen, bei dem anderen Schlitten wieder waren vier ganz schwarze Renntierochsen' (154); MS $\beta$ ắróo 'adimmī' 'kahle Flecken begannen sichtbar zu werden' (351). Einem Zaubergesang ist die folgende

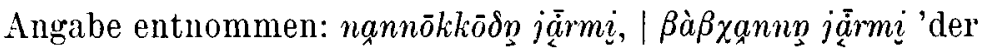
unverheiratete Jüngling weinte, | weinte im Bett' (Mat 429). Im Waldjurakischen kommen Verbalnomina mit der Endung $m m \vec{\imath}$ vorwiegend in Zitaten vor: $\mathrm{Nj}$ st'suskkăèej ṕįinñop |

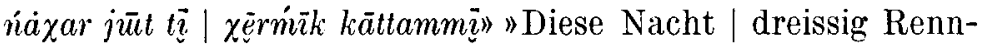

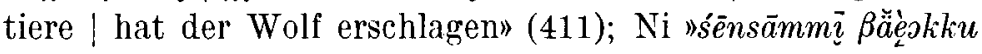

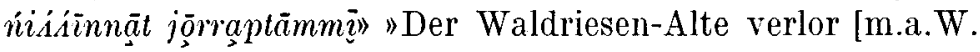
ermordetej deine Grossväter" (134). ${ }^{1}$ An das Verbalnomen kann ein Possessivsuffix treten: WNi "mań tēj kättammäàen" "Du hast mein Renntier erlegt» (132); sensāmmī $\beta \breve{a ̆ e ̀ o k k u ~ k a ̄ r \beta t p ~}$

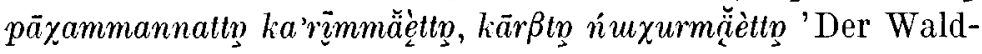
riesen-Alte hat die Lärche mit dem Wurzelende losgezerrt, er schleifte die Lärche' (134), vgl. (C) tjukū matormui-dí 'alle hatten sie [Du.] durchgeschnitten' (214).

Das mit dem Suffix ${ }^{*} m \varepsilon$ gebildete Verb a $1 \mathrm{nom}$ e $\mathrm{n}$ wird allein nicht gebraucht, nur in Verbindung mit dem Suffix $* j \varepsilon$. Bei Castrén erscheint es mit der Endung meh: numda paeusemeh 'Der Abend kam' [eig. der Himmel wurde dunkel] (227). In Lehtisalos Texten ist die Endung

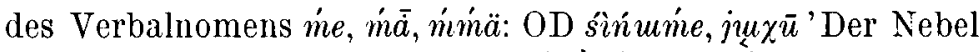

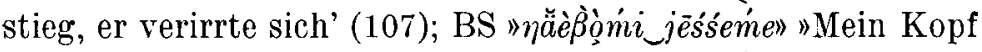
wurde schmerzhaft» (201); vgl. WNi "śëjjej jìèśmap̣» »Ich wurde hungrig» [eig. mein Herz wurde krank] (136); O njadime 'er wurde sichtbar' (172; vgl. Arch 427). In der Pluralform ist der Kehlkopfverschluss die Substitution des abgeschliffenen

1 Das Wort śēnsāmmı̄ 'Waldriese' ist ebenfalls ein Verbalnomen vom

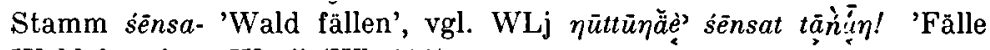
Wald für einen Weg!' (Wb 439). 
Possessivsuffixes: OD $\eta_{x}$ ajime' 'sie wurden sichtbar' (63).

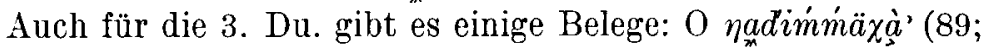
vgl. 88, 154, PD 131). - In einem Zaubergesang erscheint auch die Form der 1 . Sg. des Verbalnomens: "ḿả $\delta$ p_nimńe nadimádom' 'oberhalb von dem... Zelt kam ich zum Vor-

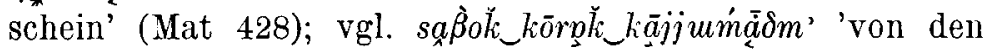
guten Renntierstieren bin ich nachgeblieben' (ebd. 425). In einer Angabe ist das Px. der 1. Pl. mit dem Verbalnomen verbunden: PD najjime $\beta \beta p^{\prime}$ ' 'wir wurden sichtbar' (63).

Im Tundrajurakischen erfüllt das $\beta \beta \bar{\imath}$-Verbalnomen in prädikativem Gebrauch dieselbe Funktion wie das Verbalnomen auf maj im Waldjurakischen, zumindest in der Ni-Mundart. Dieses Verbalnomen passiver Bedeutung kommt sowohl bei Castrén als auch bei Lehtisalo in den Texten häufig vor: (C) "tju'unjād | hasaua dōvioih, | tju'u mokodahana àmdjuvioih, | paeusemdjo jämban | sinsieukongäe malnavioih" usw. "Von oben kam ein Mann, | in der oben befindlichen Rauchöffnung | sass er, | den ganzen Abend | als Haselhuhn sang er» usw. (156). Bei Lehtisalo sehen wir in den Anfangszeilen einer heiligen Sage auch mit dem Suffix $\beta \beta \bar{\imath}$ gebildete Verbalnomina in prä-

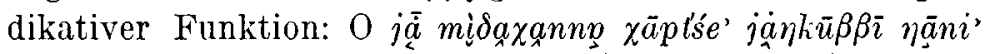

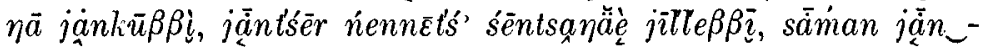

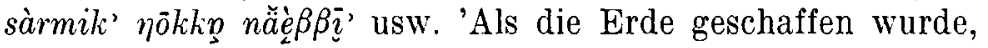
gab es keinen Tod, auch $\eta a$ [Todesgeist] gab es nicht. Der auf der Erde wohnende Samojede lebte gesund. Allerlei Tiere der Erde gab es viel' usw. (15). An das Verbalnomen kann auch das Possessivsuffix treten: (C) "jiliwaen?" "bist du noch

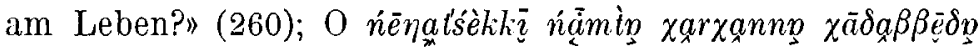
'Einmal tötete der Knabe das Mädchen mit dem Messer' (24) usw.

Es erhebt sich die Frage, ob das Suffix $\beta \beta \bar{\imath}$ in jedem Falle die Fortsetzung des Suffixes $* m a+j$ ist, oder zum Teil auf das Suffix *pa $+j$ zurückzuführen ist. Das Verbalnomen mit der Bedeutung 'tot' steht im Wb (174) in der Form $O \chi \bar{a}-в \dot{a} \dot{a} i$, WKis $k \bar{a}-p \bar{\imath}$, in Castréns waldjurakischen Aufzeichnungen finden wir die Form kaa-pui (Mat 275). Im Wb sind auch noch Kan (N) $\chi \bar{a} \beta \beta \bar{\imath}$ und OD $\chi \grave{a} \grave{\beta} \grave{i}$ angeführt, die der Herkunft nach wahrscheinlich ebenfalls mit dem Suffix *pa $+j$ gebildete 
Verbalnomina sind; vgl. noch C $k \bar{a}-v u i h$ (2), hī-vi $i_{o} i h$ (222), hãwi (25). Mit Zusammenfallen muss man also rechnen.

In der Übersetzung der Texte bezeichnet das Verbalnomen auf $\beta \beta \bar{\imath}$ die vergangene Zeit, einige Forscher hielten es auch für die Bezeichnung der vergangenen Zeit (J. Györke: NyK LI, 88-97). Dieses zustandbezeichnende Verbalnomen drückt aber keinerlei Zeitbezug aus. Hinsichtlich der Funktion ist das aus der WNi-Mundart aufgezeichnete obenerwähnte Märchen Nr. 50 aufschlussreich; darin erscheinen passivische Verbalnomina als Prädikate für die 3. Pers. Sg. Verbalnomina mit Endungen wie $m a, m a j$, jet(ta), ferner ebenfalls passivische Formen auf sotu und Verbalnomina auf $*_{\eta a}{ }^{1}$

\section{Verbalnomina auf ${ }^{*} p a,{ }^{*} p \varepsilon$}

Das verbalnomenbildende Suffix ${ }^{*} p a,{ }^{*} p \varepsilon$ hat bekannte finnisch-ugrische Entsprechungen, unter ihnen das Suffix va, $v e$ des ungarischen Verbaladverbs. ${ }^{2}$

In der Grammatik findet sich ein Gerundium auf $b$ und dieses Gerundium können wir mit dem Verbalnomen auf *pa gleichsetzen. Das Gerundium, für das es wenig Angaben gibt, erscheint bei Castrén in der Regel, bei Lehtisalo immer mit Kehlkopfverschluss am Ende: (C) »tjuku jauna $\mid$ hüveän dōb'|

1 Wie bedeutend die Rolle der Verbalnomina auf satu im Ni-Dialekt ist, beweisen auch interessante Zahlenangaben: im kurzen Märchen Nr. 37. (S. 85-86) verteilen sich die Verbalnomina in prådikativer Funktion je nach den Endungen wie folgt: sotu 28 Belege, Nomen 1, $m m p$ 2, maj 2, $\eta \eta a$ 1. - Lehtisalo hat zahlreiche Funktionen des Suffixes * $\eta a$ aufgezeichnet (AblSuff 142-144). In einem Teil der Walddialekte erscheint dieses Suffix in jedem Verb (Verbalnomen), vgl. Mat 312-313 bzw. Lehtisalos Texte. Der Gedanke liegt nahe, dass es seiner Grundfunktion gemāss ein intensiv-effektives Suffix ist, ebenso wie das Suffix r., das man aus allen samojedischen Sprachen mit viel Belegen nachweisen kann.

2 Lehtisalo hat ein Suffix ${ }^{*} p$. in einigen jurakischen Hauptwörtern und Eigenschaftswörtern nachgewiesen und dieses vom Suffix *pp. unterschieden (AblSuff 257-260). Letzteres ist entweder eine in gewisser phonetischer Lage entstandene Formvariante des Suffixes * $p$. oder kann auf eine Verbindung * $p$. $+t$. zurückgeführt werden. 
tjungae padlih" "An dieser Stelle, | wenn jemand kommt | als

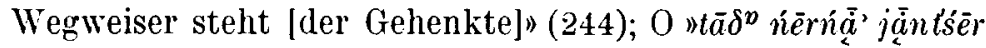

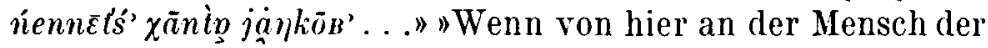
Erde kein Blutopfer hat..." (13). Der Kehlkopfverschluss weist auf ein abgeschliffenes Suffix ${ }^{*} t$.; und das aus zwei Elementen bestehende Ableitungssuffix ist tatsächlich in den Texten zu finden: (C) "jāngō-p-ta|jānda hāni!» "Wenn es

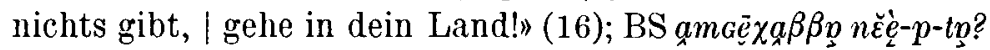
'Was mag es nur sein?' (312). Das mit * $p$. + ta gebildete Verbalnomen des Verbs 'sein' kommt oft in Verbindung mit dem

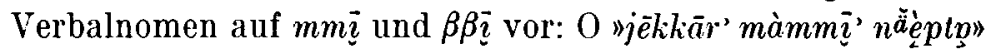
"Ich weiss nicht ob er sagte" (207); "mañi' nēenuimi' tāra $\vec{\beta} \beta \bar{\imath}$ $\eta \check{a} \dot{e} b^{\prime}$.... "wenn unsere [Du.] Tochter notwendig wäre..." (9). Das Suffix *pa kann im Gebrauch als Gerundium mit dem Suffix ${ }^{*} j \varepsilon(i)$ verknüpft werden: MS $» \gamma \bar{a}-\beta \bar{a}-j j \bar{u}-\beta$ ? » $»$ Ist

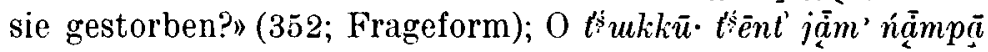
tamnn $\eta \bar{a} m t \bar{\imath}$ 'bis heute die Erde in der Hand haltend sitzt er $\operatorname{noch}^{3}(6) .^{1}$

Das zustandbestimmende Verbalnomen auf * pa nimmt auch die Possessirsuffixe an sich. Die so entstandenen Formen entsprechen nach Form und Bedeutung den Formen látcám, látvád, látvánk in den ungarischen Kodexen. (S. Károly: Igenévrendszerünk ..., 208-213, 231-236. $)^{2}$ Das Possessivsuffix bezeichnet - wie im Ungarischen - das Subjekt des durch das Verbalnomen ausgedrückten Geschehens. Solche Verbalnomina kommen - in nicht allzugrosser Zahl - fast ausnahmslos in Zitaten vor. Am häufigsten treffen wir Verbalnomina mit dem Possessivsuffix der 2. Sg. und der 3. Sg.

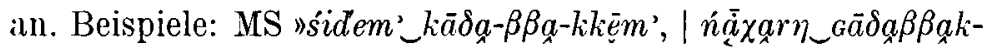
lẹ̀m'" "Zwei habe ich wohl getötet, | drei dürfte ich getötet

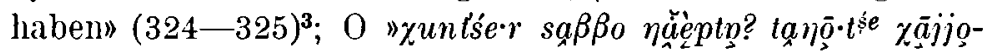

1 Das zusammengesetzte Suffix * $p a+j$ bzw. ${ }^{*} p a+t a$ entspricht im wesentlichen dem tawgysamojedischen "Charakter" des Konjunktivs

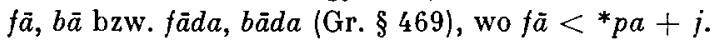

2 Igenévrendszerünk a kódexirodalom első szakaszában (Das System der ungarischen Verbalnomina in der ersten Periode der Kodexliteratur), Budapest 1956. $222 \mathrm{~S}$. + Zusammenfassung 223-234.

${ }^{3}$ Zum enklitischen Element $k k \bar{e}, k k \imath$ vgl. ALH VIII, 121. 
$\delta \bar{a}-n-n i$ ??" "Wie dürfte es gut sein? Bleiben wir [Du.] zu übersommern?" (440); (C) "äni $j a \bar{\delta}-\beta a-t \mid$ tjikeunje | njāndo mipan" "Wenn du wieder gehst | gib sie ihnen" (199); "tjukaeda häevat" "wenn du von hier fortgehst" (35), val. b. häeba (49), c. häebata (56); "indat püubat ( piubat)" "Wenn du einen Bogen für dich selbst suchst" (100); BS "téekkì mák ka "Dort in dein Zelt wenn du ankommst ..." (366); US "níвq

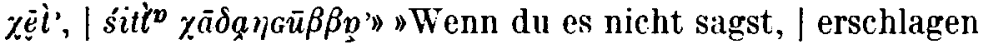
wir dich" (305); (C) "pīnjan tōBti ( tōsati'e) | tjiekahaña amahajudi!" "Wenn ihr [Du.] zurückkommt, | dann esst ihr

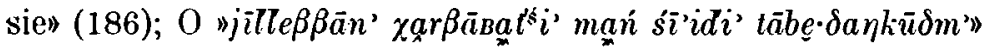
"Wenn ihr [Du.] leben wollt, bestimme ich euch" (10); - (C) "pïli püdanau | udau tatam, | tjekau juo-p-tah | opoj näeuan | sidda amdam" "Meine allerletzte | Hand gebe ich, | wenn ihr sie verliert, | in meinen einzigen Kopf | esse ich euch" (162); (C) paevude pinje | humna häebtieh? 'in der dunklen Nacht |

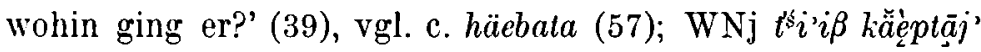

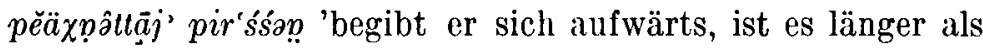

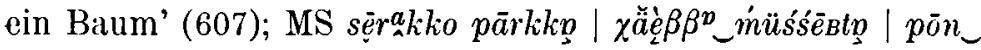

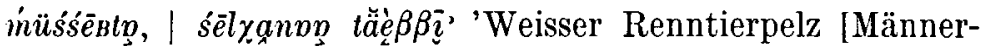
name] | ob er kurze Zeit reiste | ob er lange reiste | traf bei seinem Endziel an' (280). Es gibt auch einige Angaben für die Verbindung des Passivstammes adi- mit dem Suffix *pa: side jur meäh | tanjä adibata ( adebatu') 'zweihundert

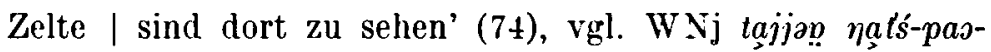
$t t \vec{a} j$ ' 'als er dort sichtbar ist' $\left(419 ; p a s<{ }^{*} p a+j\right.$.). Für Verbalnomina, die aus transitiven Zeitwörtern stammen, liegen nur wenige Angaben vor: (C) "aunanda | nieda müunje | sium häjēptah" "Einst auf dem Wege | liessen sie mich" (87); 0

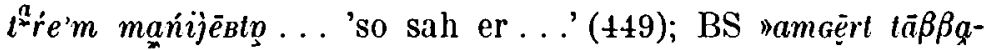

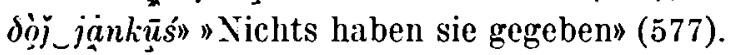

An den Akkusativ des mit dem Suffix *pa gebildeten Verbalnomens kann, nach vereinzelten Angaben, ein Possessivsuffix angehängt werden: (C) tjuonje savua jieru | ha-u-vva$m$-dah, | jilli-wā-m-dah | manatam 'Der Wirt Fuchsfellmütze | ob er gestorben ist | oder ob er lebt, | gehe ich sehen' (246);

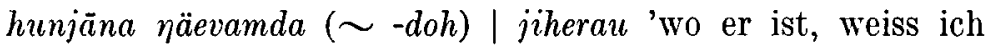




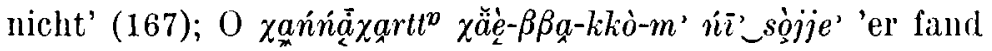
nicht, wo er hinauskommen konnte' $(170) .^{1}$

Für den Lativ des mit dem Suffix *pa gebildeten Verbalnomens, dessen Bedeutung derjenigen des ungarischen Verbaladverbs auf ván, vén entspricht, sind ebenfalls nicht viele Angaben zu finden: (C) "anīna tō-ban | tjikēnja sium idanjin" "Wenn ich ein anderes Mal komme, | dann hängst du mich

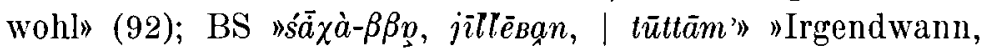

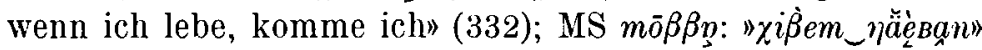
'Er sagte:' "Wer-ich sein mag" (279). An den Lativ wird in vereinzelten Belegen das Px. der 2. Du. angehängt: $O$ "pūnnp

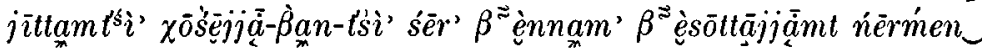
sì holet, stosse Halbhund als Stellvertreter von dir durch das

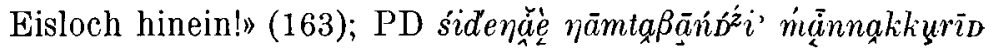
$\chi \underline{i n g} \bar{a}$ 'er höhlte [den Baum] zu der Grösse aus, dass sie darin zu zweien sitzen können' (122). Vgl. noch die folgende stehende

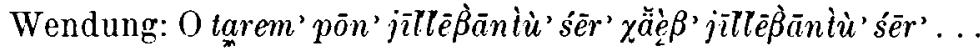
'Während sie so lange Zeit lebten, kurze Zeit lebten . . ' (11). - In einer Angabe liegt der Lativ in prädikativer Funktion vor: (C) tjüöriej, | jāndo njāuotajā | njāngaeda häeuvan 'Er' begann zu schreien, | die dünnhaarigen Spitzhunde kommen galoppierend | auf ihn zu' (231-232).

Es gibt mehrere Angaben in Castréns Texten, wo dem Laltiv des mit dem Suffix * $p a+n a$ gebildeten Verbalnomens ein Possessivsuffix angehängt wird: $\| j i l \bar{e}-b-n a \bar{n}(\sim j i l i e b n \bar{a} m) \mid$ sit'eh hōptsum, | hä-b-nan $(\sim-n \bar{a} n) \mid$ tjikī $j a \bar{n} a \mid$ sēbemsi beänor!» "Wenn ich lebe, | finde ich dich, | wenn ich sterbe, | an dieser Stelle | beginne ich zu verfaulen" (79); "amga jānd | taeupananda ... »an irgendeinen Platz | wenn du gerätst . ..»(211);

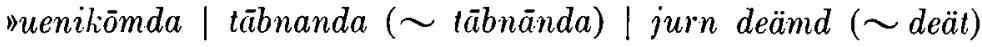

${ }^{1}$ In einigen Sätzen erscheint das Suffix *pa $(+j$.$) - als eigenartiges$ determinierendes Element -- im Akkusativ eines Pronomens bzw. No-

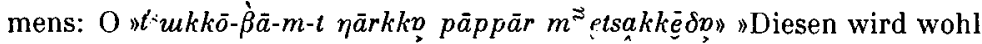

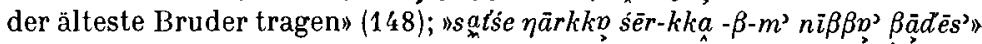

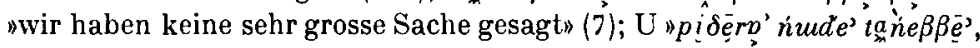
$a_{A} m G \bar{e}-\chi a-\beta-m^{3} k \bar{a} \delta a \eta G u^{\prime}, \chi \bar{o} \eta G u^{3}$ »Eure Jungen sind da, sie werden etwas fangen, finden" (Mat 353). 
mitjon, | nibnjānda ta'ah | punjān tānanōd!» "Seinen Hund wenn er (ihn) gibt, | hundert deiner Renntiere gib, | gibt er nicht, | treibe sie zurück" (180); "amgae äebnāndi'?" "Was mögen sie [Du.] sein?» (14); "satji mungoh | tōbnāndo'oh tanjeh hānon!»"Wenn heftige Pfeile kommen, dann stirb!» (201).

Aus dem Stamm mańi $+j \bar{j} \bar{e}$ - stammen folgende Lativfor-

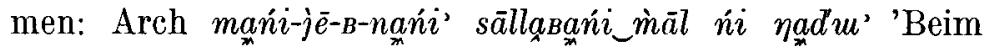
Umherblicken ist das Ende meines Eises nicht sichtbar' (430);

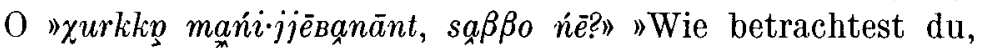
[ist sie] eine gute Frau?" (443). - Das Suffix des Lativs $(+$ Possessivsuffix) tritt in nicht sehr vielen Belegen an das mit dem Suffix *pa + ta gebildete Verbalnomen; anstatt des Suffixes ${ }^{*} t a$ findet sich oft ein Kehlkopfverschluss: (C) »amgam nüptāni ( nubtāni)?) "Warum stehen wir [Du.]?" (134);

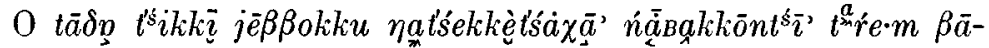

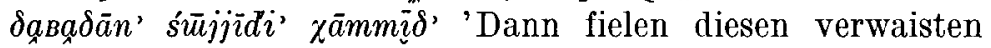
Kindlein, während ihre ältere Schwester sie so aufzog, die

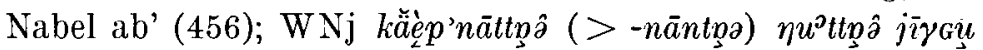
'Wenn er geht, gibt es von ihm keine Spur' (607).

Verbalnomina a uf $\not{p} i, m p i$. Verbalnomina auf $\dot{p} i$, die meistens von transitiven Verben stammen und die auch Possessivsuffixe an sich nehmen können, kommen in den Texten ziemlich selten vor. Bedeutend mehr Angaben besitzen wir für das Verbalnomen auf mpi, wo das Suffix $p i$ mit dem zustandbezeichnenden Suffix $m$. verbunden ist. Die Angaben bestätigen jene kontinuative Bedeutung der mit $p i$ gebildeten Formen nicht, auf die sich Castrén berief und die nach seiner Meinung ein solches Suffix enthaltende Formen dazu geeignet macht, die "gegenwärtige Zeit» auszudrücken (Gr. § 476). In der Grammatik haben wir im Paradigma des Verbs mit der Bedeutung 'bedecken' den Wandel $b i \sim b a$ vor uns $(\S 495)$. In den Texten unterscheiden sich aber die Verbalnomina auf $p a(m+p a)$ und die auf $p i(m+p i)$ deutlich voneinander: $(\mathrm{C})$ "eäsodabnani ( $\sim$ üäsoumbanani) $\mid \bar{o} b$ neäsohani', | müusieu(m)banani' | üäsohani!» "Wenn wir uns lagern, | einmal lasst uns lagern, | schlagen wir auf unserer

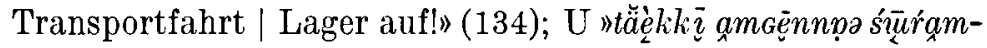




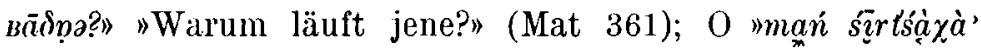

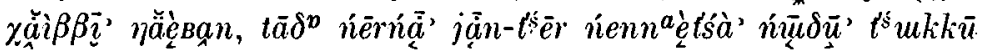

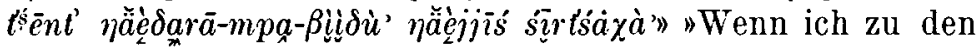
Tschuden gegangen wäre, dann hätten von da ab die auf der Erde wohnenden Menschen ihre Kinder bis jetzt zu den 'Tschuden geschickt') (157).

Um die Herkunft des Suffixes $\not \dot{p} i$ zu erklären, müssen wir in Betracht ziehen, dass das konsonantische Element des Suffixes ein mouilliertes $\dot{p}$ ist. Die folgende Angabe ist lehr-

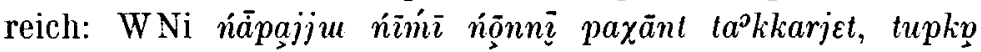
$v^{\prime} \bar{a}^{\prime} m-\dot{p} \bar{o}-\eta \eta \eta_{\supset}^{\prime}$ 'Das Weib des älteren Bruders versteckte sich in dem einen Türwinkel, hält eine Axt in ihrer Hand' (103; vgl. 142). Im Suffix - $\dot{p} o$ - ist das mouillierte $\dot{p}$ auffallend: $\dot{n}^{\prime} \bar{a} m-\dot{p}^{-}-$ ist ein sekundärer, mit dem Suffix $* j+\beta$ gebildeter Passivstamm, die Mouillierung des Elementes $p$ wurde durch das Suffix $* j$. verursacht. Unter Beobachtung dieses Stammes können wir das Suffix $\dot{p} i$ auf eine Verbindung ${ }^{*} p \varepsilon+j$. zurückführen. Das Suffix ${ }^{*} p \varepsilon$ ist das palatale Gegenstück zum Suffix ${ }^{*} p a$ wie das Suffix ${ }^{*} n \varepsilon$ dasjenige von ${ }^{*} n a$ ist, was wir weiter unten sehen werden. Allein wird weder das Suffix *pe

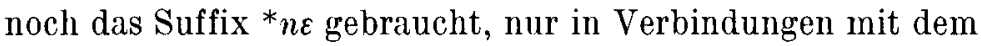
Suffix ${ }^{*} j .:{ }^{*} p \varepsilon+j .>p i,{ }^{*} \varepsilon \varepsilon+j .>n i$. Der passivische Verbalnomenstamm auf $\dot{p} i$ erscheint in den folgenden Formen der 3. Sg.: (C) meäkanda taevioih | tĭda äedpi 'er kam zu seinem

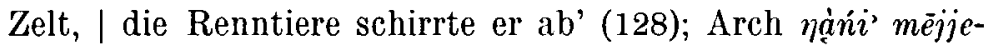

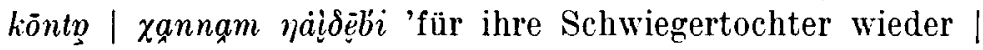

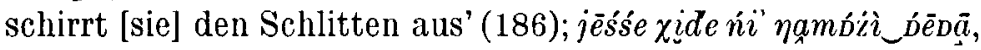

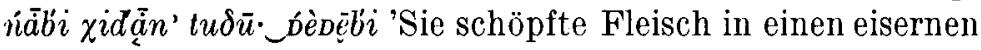
Napf, in den anderen Napf schöpft sie Fett' (437-438). Das Verbalnomen nimmt das Possessivsuffix der 3. Person

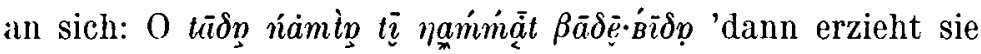
ihren Kameraden [d.h. das Kind] mit dem Renntiereuter' (446); (C) siu jāle nikalpidi 'sieben Jahre reissen sie [Du.] ihn' (43). Anstatt des abgeschliffenen Possessivsuffixes sehen wir den Kehlkopfverschluss: MS šide $\beta^{z}$ èsaktiò jēśse jinnnem' $\beta$ sènppì? 'Zwei Greise ziehen gegeneinander an einer Eisenschnur?' (592). Es finden sich sehr wenige Angaben für die

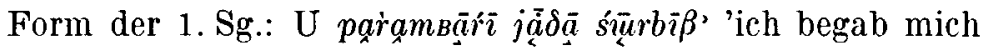


eilig laufend fort' (Mat 360); Ar'ch s'ämi 'mäsabisism' 'Ich wasche mein Gesicht' (437).

Bedeutend mehr Belege besitzen wir für das Verbalnomen auf $m p i$, das aus transitiven, seltener intransitiven Verben stammt: (C) "njana parombim" "Ich habe grosse Eile" (93); BS

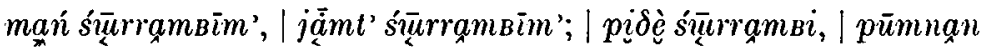

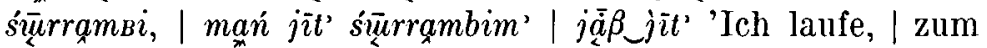
Meere laufe ich | er läuft, | läuft hinter mir her. | Ich laufe in das Wasser, | in das Wasser des Meeres' (289); U śịirambintpa

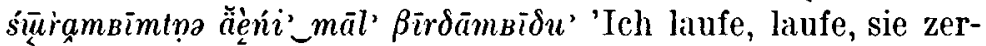
kratzten ganz meine Füsse' (Mat 360). ${ }^{1}$ — Es gibt zahlreiche Beispiele für das Verbalnomen vom Stamm na'am-: MS pāl-

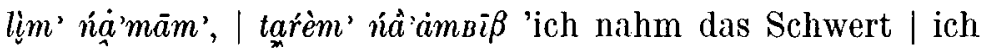
halte [es] in meiner Hand' (347), vgl. ńá'amsim' (Mat 359); (C) njämbi-ua ( njāmbina) 'wir hielten es fest' (164); "njāmbira!" »haltet [es] fest!» (161). In der Form der 3. Sg. erscheint zum Teil der Verbalnomenstamm, zum Teil dessen mit Pos-

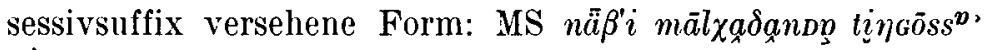
riá 'ámBi 'am einen Ende hält | der Tunguse fest' (346); Kan laurmdy niáa $m b i \delta p$ 'er nimmt seine Treibstange in die Hand' (218); (C) njämbido'oh 'sie halten es fest' (161); PD ńámbixi'

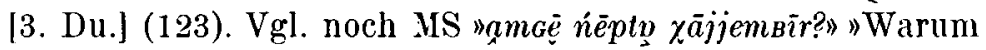

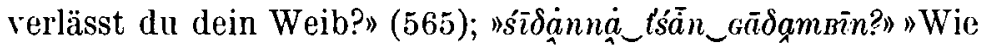
viele Male tötest du uns?» (561).

Die Angaben beweisen, dass die Verbalnomina auf $\not \dot{p} i$ (mpi) kein Zeitverhältnis bezeichnen, wie auch die ebenfalls nominal gearteten Verbalnomina mit dem Suffix $p a$ (mpa) keine Zeitstufe ausdrücken. Zu der Erklärung, dass das Suffix $\dot{p} i$ (mpi) kontinuative und präsentische Bedeutung hat, mögen die zustandbezeichnende Funktion und die zum verbalnominalen Prädikat gehörenden temporalen Bestimmungen Anlass gegeben haben. Die folgenden beiden Zeilen eines Gesanges sind in dieser Hinsicht aufschlussreich: (C) pōn mimbitie? | haeu mimbitie? 'Ging er lange | ging er kurze Zeit?' (45). In den Varianten $b$ und $c$ erscheint das Verbalnomen auf

${ }^{1}$ Die Partikel ṭ̣a, die in einigen Dialekten sehr oft als verstärkendes Element vorkommt, ist ihrer Herkunft nach nichts anderes als das Px. der 3. Sg., eventuell der 2. Sg. 
* pa in derselben Bedeutung: mimbatie (53), mimbata (60), leig. ist sein Gehend-sein lange? ist sein Gehend-sein kurze Zeit?] vgl. MS 264 usw.

\section{Das Verbalnomen auf ${ }^{*} n a,{ }^{*} n \varepsilon$ mit potential-konditional-optativer Bedeutung}

Beide Formen des Suffixes uralischer Herkunft sind im Kamassischen rorhanden ( $D-J$ 170) und von den finnischugrischen Sprachen im Ungarischen.

Im Jurakischen erscheint das mit dem Suffix * ${ }_{n} a$ gebildete Verbalnomen in Zitaten, in der Sprache der Lieder und der Sagen. Das Suffix wird oft mit dem Suffix $* j \varepsilon(i)$ des Konjunktivs verbunden, sowie mit dem enklitischen Element $k k e$, $k \cdot k \underline{i}$, ferner mit den Irrealität bezeichnenden Suffixen und im Waldjurakischen auch mit dem Suffix ${ }^{*} \eta a^{1}$ Die Possessivsuffixe und die Kasusendungen werden daran angeschlossen, in erster Linie das Lativsuffix. Die Zahl der Angaben ist nicht

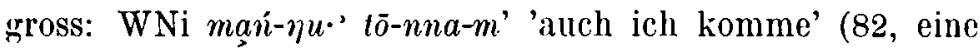
stehende Wendung im Märchen Nr. 37); WLj käę $\beta \chi a t ~ \eta a ̄ m t-$ $n a-j j \varepsilon \cdot m$ ? 'Setze ich mich neben dich?' (102); WNj tśièptī

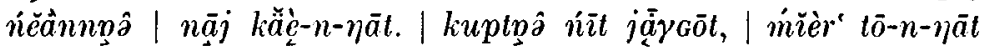
'Morgen | wieder gehe ich. | Lange bin ich nicht fort, | bald

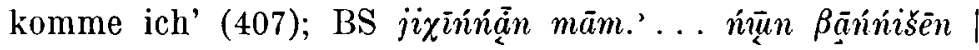
$\chi \bar{a} n-b a-n n a-k k \bar{e}-m$ ' 'Ich dachte... zu meinem Solın Hans | werde ich mich wohl begeben' $(572-573)$; W Ni $" t^{\prime s} i^{\circ} k k i$

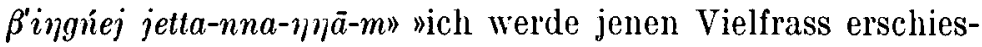

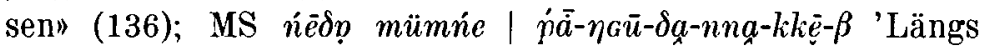
der Schlittenspuren | begebe ich mich wohl [zu schreiten]'

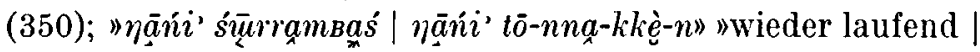

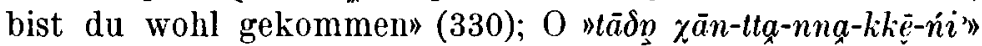

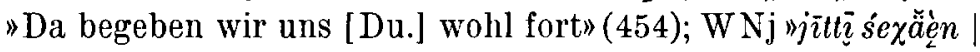

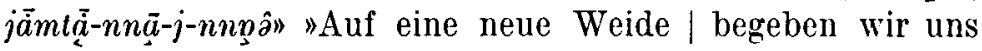
auf die Reisen (412). Viele Angaben besitzen wir für die Form

${ }^{1}$ Lehtisalo hat das Suffix -nna-, -nno- $(-t t a-)$ auf das Suffix *n!ș zurückgeführt (AblSuff 238-240). 
der 3. Sg.: (C) side juongatie | heämzawaej | sisju hai-nā'aus ihren beiden Mundwinkeln | blutig | der Schaum strömt [geht]' (214; auslautendes $\bar{a}<{ }^{*}(n) a+j$.). Bei den Formen des als Prädikat gebrauchten Verbalnomens erscheint ein Possessivsuffix bzw. Kehlkopfverschluss: O "néerĩ tsóñé-nna-

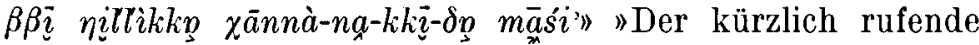
Teufel hat sie wohl weggeführt» (47; das Suffix *na erscheint auch in dem als Attribut gebrauchten Verbalnomen auf $\beta \beta$ i);

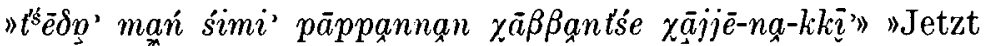
haben mich wohl meine jüngeren Brüder verlassen um zu

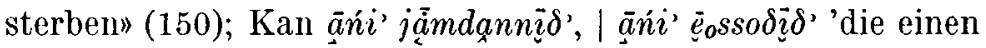
sind auf die Umzugsreise gegangen, | die anderen haben gelagert' (225).

Das Verbalnomen mit nominaler Bedeutung (eine solche Bedeutung hat es auch in prädikativer Stellung) wird im Satz als Subjekt, Objekt und Umstandsbestimmung gebraucht:

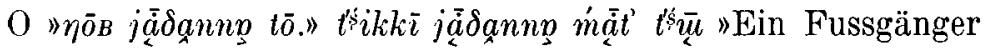
kam.» 'Der Fussgänger trat in das Zelt hinein' (10); Kan

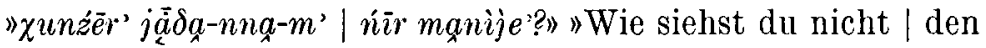

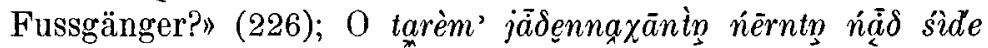

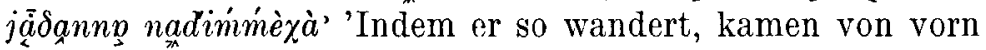
zwei Fussgänger in Sicht' (88). -- Für die mit Kasussuffixen versehenen Formen des mit dem Suffix *pa + na gebildeten Verbalnomens haben wir weiter oben Beispiele gesehen (S. 178).

Die Fortsetzungen der Suffixverbindung $* n \varepsilon+j \varepsilon$ lauten im Jurakischen $n i \sim n i \sim n e \sim n^{\prime} \dot{a}^{1}$ Die spärlichen Angaben stammen mit wenigen Ausnahmen aus Castréns Texten: (C) "hanamda pōderi-ni-m" "Deinen Schlitten werde ich ausspannen» (47), "niedamda hō-ni-n!" »Ein Weib für dich finde!" (94); "tānsa nieve hādakau | obum njüda tāri-nji-n | njātsadan tārinjin!" "Mutter der Eidechse, meine Grossmutter | einen

${ }^{1}$ In Castréns Paradigmen erscheint - wie bereits erwähnt - das Suffix $n i$ als eine Variante des Konjunktivsuffixes $j(i)$; es ist auch im Jenisseisamojedischen vorhanden (Gr. S. 501-512). Im Selkupischen finden sich in den Paradigmen des Verbs mit der Bedeutung 'ich zünde an' die Suffixe ne und ni: Ket Tschl. ne (Mat 205-206, 213), N ni (ebd. 196, 210). In manchen Angaben erscheint die Verbindung $k u+n e \mathrm{bzw}$. $k u+n i$ (ebd. 209, 216\}. 
Knaben nur gib | zu meinem Kameraden nur gib!» (303); "niseända jān | hōrjini'e ( hor-nji-ni')" "in deines Vaters Land | gehen wir [Du.] zu versuchen" (227); "jiles hō-nji-ra'ah mäjenda hōnjira'ah" »im Leben werdet ihr finden | eure Leiden

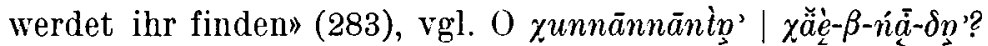
'Wohin | begabt ihr euch?' (505, Zaubergesang). Vereinzelte Angaben haben wir auch für die Formen der 3. Sg. und 3. Pl.: MS éorọọ "tieda jānguh | ni-nje-ki jileäh» »jetzt sind sie weg | vielleicht leben sie nicht" (94). Dem von einem transitiven Verb stammenden Verbalnomen wird das $\mathrm{Px}$. der 3. Sg. angehängt: "tjukī hāsaua | amgädanda | tēkutsini | haije-njā-kide?»» »Dieser Mann | warum | unsere Renntiere | mochte er lassen?» (136). Als Subjekt haben wir das mit den Suffixen $*_{n a}$ und ${ }^{*} n \varepsilon+j \varepsilon$ gebildete Verbalnomen in einer stehenden Wendung: BS

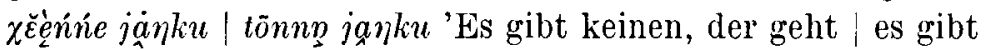
keinen, der kommt' (570), vgl. (C) "janāna | hāji-njā-do jānguh" "in unserem Lande | ist niemand zurückgeblieben" [eig. ihr Zurückbleiben (Potentialis) gibt es nicht (Konj.)] (213). In Castréns Texten kommt der Lativ des Verbalnomens mit Possessivsuffix vor: humna häe-b-njān-da? 'Von wo ging sie?' (164); tanja jile-njāda ( jile-njahan-do) 'Während sie dort leben... (83; vgl. 84, 174, 185, 249); vgl. noch MS ımań

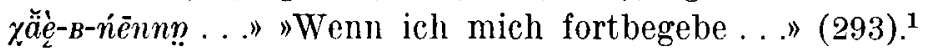

Verbalnomina mit dem Suffix ${ }^{*}{ }^{s}$.

D as Verbalnomen a u ${ }^{*} s^{\prime} a,{ }^{*} \dot{s} \varepsilon$

Castrén behandelte die Modi im zweiten Kapitel der Grammatik. Zu seinen Feststellungen fügte er folgende Bemerkung hinzu: "Es ist jedoch möglich, dass irgendein einzelner Modus

${ }^{1}$ Castrén behandelte - mit Vorbehalt - das enklitische Element rawa, lawa (in den Texten -raha-, [L] -raxa-) als Zeichen des Optativs (Gr. \$ 470). Dieses Element, dessen Bedeutung 'wahrscheinlich, vermutlich, gleichsam' usw. ist, kann ebensowenig als Moduszeichen betrachtet werden wie das ebenfalls enklitische Element $k k \bar{e}, k k \underline{i}$. 
von mir entweder übersehen oder unrichtig aufgefasst ist» ( $\$ 467)$. Ein solcher "übersehener Modus» ist im Jurakischen das Verbalnomen auf ${ }^{*} s a,{ }^{*} s \varepsilon$, dessen Bedeutung derjenigen des finnischen Konditionalis (in einzelnen Fällen des finnischen Potentialis) und dessen ${ }^{*} s$-Element auch dem $s$-Element des finnischen Konditionalis gleichgesetzt werden kann. Die Bedeutung der jurakischen Konditionalformen könnte man am genauesten mit den entsprechenden finnischen Konditionalformen bestimmen. Der jurakische Konditionalis kommt in Zitaten, in gesprochenen oder nur gedachten Reden der Personen, in Fragen, Verneinungen vor.

D a s S uffix *'sa erscheint an sich ziemlich selten: (C) manj darem mam'ah: | "njeveau haroa-sa-dah, | hunjan garoasa-da'ah, | saeusi haroasada'ah" 'Ich sagte so: | Meine Mutter wolltet ihr ["haluaisitten] | wohin wolltet ihr, | die Blinde wolltet ihr, | wohin wolltet ihr?» (283); "tjuki munga' mana-sa-n!" "Diese Pfeile siehe ["näkisit»]! (87), vgl. T "jàstarmmārn mannésa-mm»!" "Sieh doch die Mauserstelle an!" (Mat 417); $\mathrm{N} » t \bar{a}$

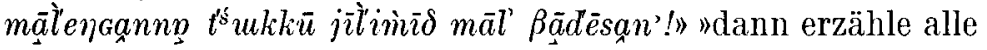

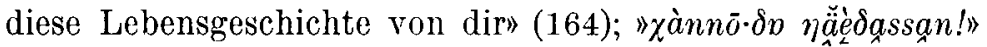
"Befreie die Schlitten von den Geschirren!" (444); BS "lop

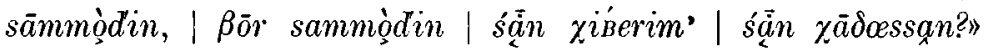
"Heide Samojede | Dieb Samojede | wieviel Menschen | wieviele hast du getötet?" (323); PD noppòi $\beta \bar{a} \delta a j i j i$ ' I "mañni'

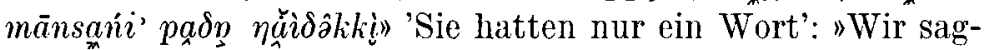

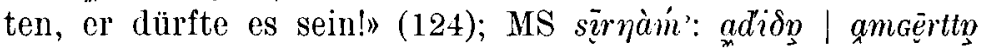

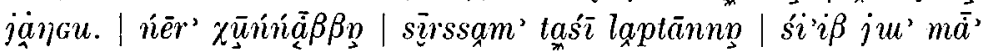
'Ich schaue: | nichts ist zu sehen. | Kürzlich wohin ich blickte, | unten in der Niederung | sind siebzig Zelte' (294). In einer Angabe aus dem WNi-Dialekt erscheint das Suffix *'a in

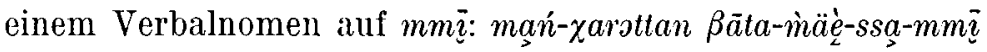

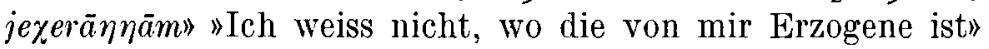
(141). In verneinenden Fügungen erscheint das Hauptverb oft mit dem Konditionalsuffix und Kehlkopfverschluss am Ende: (C) njivioida manas' 'er sah [es] nicht' (179); O

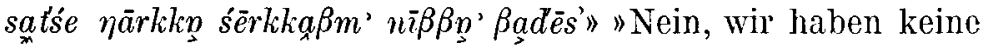
grosse Sache gehabt” (7).

Das Suffix * ${ }^{*} a$ kann mit dem Suffix $* j \varepsilon(i)$ des Konjunktivs 
verbunden werden, und so sind die Varianten $s s \bar{a}$ (und mit sekundärer Affrikate) $t s \bar{a}$ entstanden: BS »śáa $\grave{a}^{\prime}$ mannetsām'?" "wann habe ich gesehen?" (362), vgl. "sáxam_mannętsā $\beta$, I tartśem_mannetsam??" "Wann habe ich [gesehen] | solches gesehen?» (364); (C) "han-sā-ka-ua'ah" "Wir werden wohl bringen"

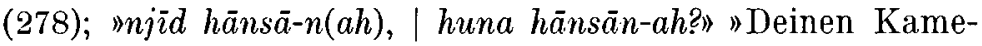
raden brachtest du, | wohin brachtest du [ihn]" (297); O "xat-

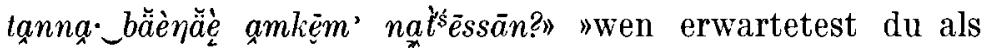

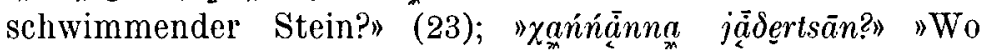
gingst du?» (26).

E i n $\mathrm{S} u \mathrm{ffix}{ }^{*}{ }^{\prime} \varepsilon$ an sich lässt sich nicht nachweisen, sondern es wird ebenso wie das Suffix ${ }^{*} n \varepsilon$ und ${ }^{*} p \varepsilon$ mit dem Suffix des Konjunktivs $* j \varepsilon(i)$ verbunden. Das Suffix $* j \varepsilon$ hat auch in diesem Fall die sekundäre Mouilliertheit des Konsonantenelements im Suffix und - wie in vielen Fällen seine Entwicklung zu einer sekundären Affrikate verursacht. In den Suffixvarianten des jurakischen Konditionalis $s \dot{s}, \dot{s} \bar{e}$,

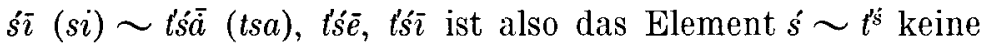
direkte Fortsetzung eines uralischen $* \stackrel{c}{*}$. Da die Zahl der Belege ziemlich gering ist, behandle ich die von intransitiven und transitiven Verben stammenden Formen zusammen: (C) "manj häe-r-tse-m!» "Wenn ich ginge!" (74); "lānārtsem" "Wenn

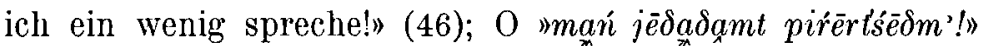
"Wenn ich dir mal eine Suppe kochte!" (160); "nin amaé $t^{\prime} \geqq k-$ kion' $t \bar{a} s \bar{s} \delta$ ? " "Meine Brüder, warum hast du sie hierher gebracht?" (48); W Nj tāśapt? 'hast du sie gebracht?' (Wb 476);

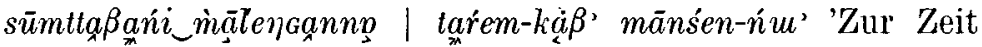
meines Gutwerdens | so sagtest du ja' (Mat 425, Zauberge-

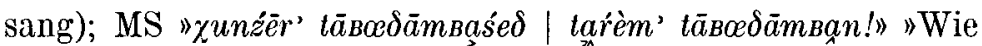
du befohlen hast, | so befiehl!» (267); BS "ńē pāppakkkōmtp | nennp' śänśttīrı "Deine jüngere Schwester | schonst du sehr" (357). - Die Formen der 3. Sg., in denen der Verbalnomenstamm des Konditionalis erscheint, behandle ich zusammen mit den formal identischen Formen der 3. Sg. des "Präteritums».

Sehr interessant sind die Verbalnomina des Konditionalis vom Stamm min- 'gehen', die - manchmal mit dem verbalnomenbildenden Suffix * ma verknüpft — dem heutigen Sprach- 
grefühl gemäss die Funktion eines Attributs in einer Fügung

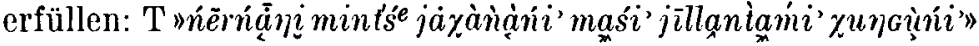
"Weiter vorwärts in dem Lande, in das wir gehen werden, finden wir vielleicht einen lebenslänglichen Aufenthaltsort» (Mat 420); (C) "manj hāntam | niernjā, | minsima jān hāntam» "Ich gehe voraus, | zum Bestimmungsort ich gehe" (246). Es gibt Angaben für den mit Possessivsuffix versehenen Lativ des Verbalnomens: (C) "piodar hānj | njānanda hānj, | minsinda jān | taeuvāda | sauvvambouna!»»Du gehe, | mit ihr gehe, | in das Land, | in das sie geht, | bringe sie | gut!" (235); taŕèm'

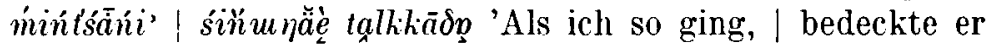
sich mit Nebel' (Mat 428; Zaubergesang); PD tā nülttāsáàni'

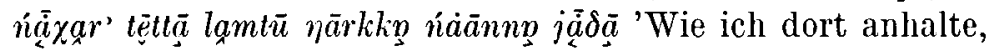
kommt der älteste der drei lamtü- Hausherren zu mir gegangen' (Mat 406). - Dem Verbalnomen werden die Suffixe $j i$ bzw. ni $i$ und das Possessivsuffix angehängt: $O$ "maśi miniśsi-

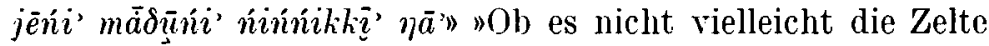
sind, zu denen wir [Du.] unterwegs sind" (447). Ein solches Verbalnomen tritt im Satz auch als Subjekt auf: (C) "min$s i-j \overline{-}-n e^{\prime}$ | härioih" "Die zu denen wir [Du.] gingen, | sind tot" (248); "sit pisidavioi | adisijido' tallih" "Hier hast du für das Sehen | derer, die über dich lachten» (198); vgl. U »āñ $i^{\prime} \dot{m a} \bar{a} k-$

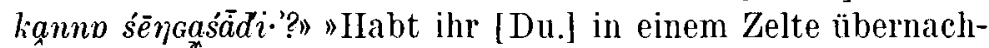
tet?" (Mat 366).

In einem Gesang begegnet uns der Lativ bzw. Lokativ des mit dem Suffix ${ }^{*} \dot{s} \varepsilon j+p a$ gebildeten Verbalnomens in prädikativer Funktion: (C) mansixān ( $\sim$ mansiuāna) madm' $(\sim$ mam'ah $): \mid$ niembāpau | mipnan $(\sim \operatorname{mipnjāna)~..."»Ich,~}$ ich sage [fig. etym.]: | meine jüngere Schwester | wenn ich

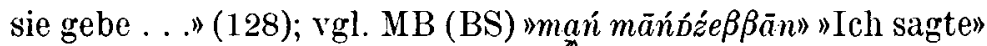
(Mat 377). In einem Gerundium wird das zusammengesetzte Suffix ${ }^{*} l \varepsilon+p .+{ }^{\prime}\left({ }^{*} t\right.$.) mit dem Verbalnomen des Kon-

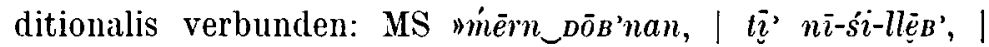

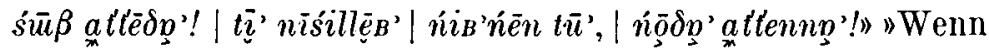
ich bald komme, | wenn die Renntiere zu kalben beginnen, | erwartet mich! | Beginnen die Renntiere zu kalben, | und ich komme nicht, | wartet nicht!" (293). - 
Für das Verbalnomen mit dem zus a mmengesetzten Suffix $p$. $+s a+j$. liegen nicht viele Angaben vor: (C) »hümjäna paeuseme | tjikẹn üäsadōn! | harna tō-btsa-ke-m" "Wo es dunkel wird, | dort schlage das Lager auf! | Selbst werde ich kommen" (247; vgl. 236); "jin douãna | harn dōptsale eu" "Wenn ich Lust habe, | komme ich wohl selbst"

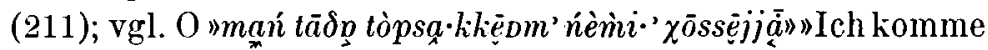

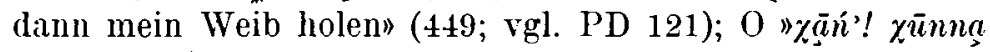

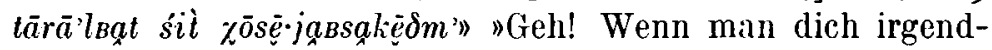
wann braucht, werde ich dich wohl holen kommen" (44);

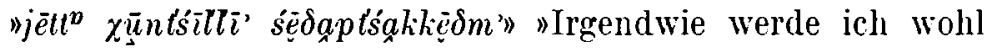
nähen" (149); (C) "hānā-ri-ptsa-kae-ua'ah ( Thān-sã-ka-ua'ah)» "Wir werden wohl bringen" (278).

Auch für das Verbalnomen mit dem Suffix $p .+s \varepsilon+j$. besitzen wir nur wenige Angaben: (C) "tjukī jäd | nisenat $j \bar{a} n$ | ānin gäe-ptsi-ni'eh" "Von diesem Platze | in das Land deiner Eltern | wir wieder uns begeben" (224). -- In den Formen der 3. Sg. sehen wir den Verbalnomenstamm mit dem enklitischen Element kike, liki und - bei einigen Verbalnomina mit transitiver Bedeutung -- mit dem Possessivsuffix ver-

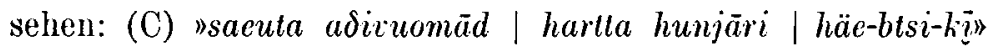
"Nachdem er seine Augen geöffnet hat, | selbst irgendwohin | geht er wohl" (45); "njär $(\sim$ njāra $) \mid$ sit täbatä-ptsc-kith" "dein

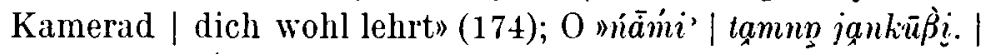

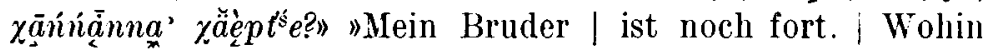

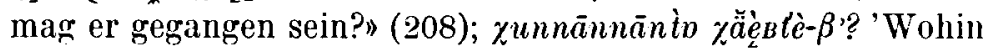
sollte er wohl gegangen sein?' (470; Zaubergesang); "pārk-

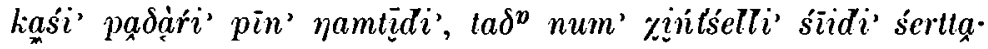
ptsakkin "ihr setzt euch nackend hinaus, dann wird wohl num

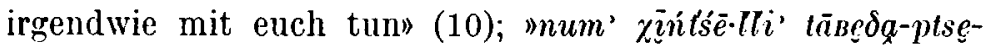
like- $\chi e j i \bar{u}-\delta p$ " "num befiehlt sie [Du.] wohl irgendwie" (7);

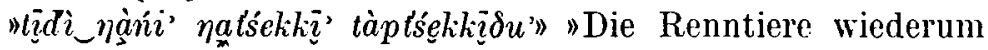
bringen die Knechte" (451). Als Gerundium erscheint das

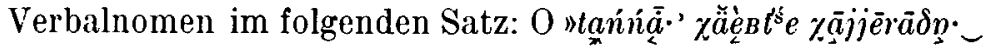

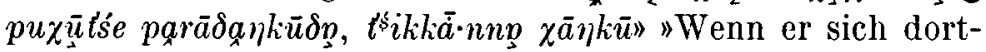
hin begibt, verbrennt ihn die Sonnenalte. Da stirbt er" (44). ${ }^{1}$

1 Der eventuelle Zusammenhang (vgl. ALH III, 305-306) des Suffixes pts des jurakischen Konditionalis mit dem Suffix pč des lappischen Konditionalis ist eine strittige Frage. Nach Erkki Itkonen hat dieser 
Zum Verbalnomen des Konditionalis können in seinem nominalen Gebrauch die auch die Funktion des Gerundiums erfüllende Stammform des Verbs 'sein' und Possessivsuffixe

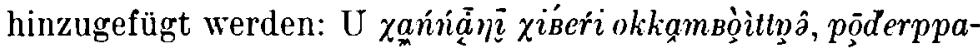

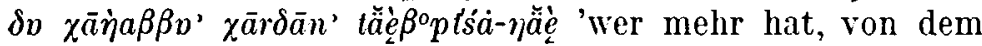
bitten wir ein Zugrenntier, um zum Dorf zu gelangen' (Mat 350); 0 "mépt'semi " jāmí t'sán "s "Sieh, hier ist unser [Du.]

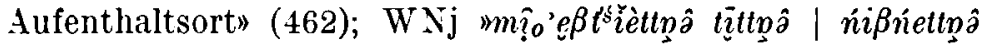
$m \approx \grave{s} s$ ') "Die von ihm gehaltenen Renntiere, | wie hielte er sie nicht" (419). Ein Konditionalsuffix enthalten die Wörter verbalnominaler Herkunft tundrajur. $\chi \bar{a} p t^{\prime} \bar{s}^{-}$' 'Tod', WP kāpśa id. (Wb 175) und tundrajur. jit̀ êpt's' 'Leben', vgl. WP diAAípse $\eta$ dèa 'Wohnort' (Wb 128). Das $\eta$ im Auslaut ist auch ein verbalnomenbildendes Suffix, sei es ein ursprünglicher oder cin sekundärer Laut $\left(\eta<{ }^{*}-n\right)$. Die Auffassung, dass weder das Leben, noch der Tod als wirkliche Zustände betrachtet werden kömnen, ist wohl in der Glaubenswelt der Juraksamojeden verwurzelt. O $\chi \bar{a} s s n$, OP $\chi a s s œ j j \bar{\imath}, \mathrm{WNj} k \bar{a} s-$ snâ 'gestorben, selig' sind Verbalnomina, mit dem Suffix *sa bzw. ${ }^{*} s a+j$. gebildet. Das Wort $\chi \bar{a}$ вạa, WP käpáei, Kis kiapi 'gestorben, tot' ist ein Verbalnomen auf ${ }^{*} p a+j$, das konjunktivisch-passivischen Sinn hat. Diese jurakische sprachliche Erscheinung erinmert daran, dass zur Bezeichnung des Begriffes 'mortuus' in einigen finnisch-ugrischen Sprachen ein Verbalnomen mit Präsens-Bedeutung gebraucht wird (vgl. Paavo Ravila: JSFOu 59, 4 ). ${ }^{1}$

Konditionalis, der vom Ter-Lappischen bis zum Lule-Lappischen nachgewiesen werden kann, im vorigen Jahrhundert im Inari-Lappischen noch gelebt (FUF XXX Anz. 8 und XXXVII, 127). Mikko Korhonen (MSFOu 143, 126-127) lehnt die Möglichkeit des Zusammengehörens ab und führt das $p$-Element des lappischen Konditionalzeichens auf ein Element ${ }^{*} k$ zurück. - Eines ist sicher: im $p$-Element des juraksamojedischen Suffixes ist das urtümliche uralische verbalnomenbildende Suffix * ${ }^{*}$. bewahrt.

${ }^{1}$ Zur nachdrücklichen Hervorhebung der Irrealität braucht man im Jurakischen - in Ermangelung eines besseren Fachwortes - die "Determinierung» mit Ableitungssuffixen. Dies besteht darin, dass die die Irrealităt bezeichnenden Suffixe nicht nur im Prădikat erscheinen, sondern auch an andere Satzglieder angehängt sind: mpoppó̀i ṕi-kkó-tśa-

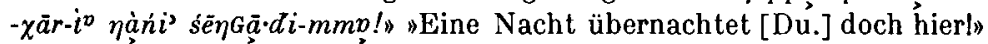
(444). Das Prădikat ist eine Konjunktivform. Im Wb begegnet im Stich- 
V e r b a ln o m in a a uf $s \bar{o}, s \bar{u}, t s u, s ́ s \bar{o}, p s u, p t s \bar{u}$. Die verbalnomenbildende Suffixe $s \bar{o}, s \bar{u}, t s \bar{u}$ stammen aus einer Verbindung ${ }^{*} s \sim s \varepsilon(+j)+\beta$, die Suffixe $s^{\prime} s \bar{o}, p t s \bar{u}$ aber aus einer Verbindung ${ }^{*} p$. $+s^{\prime} a j \sim \dot{s} \varepsilon j+\beta$. Die mit diesen Suffixen gebildeten Verbalnomina sind also passive Formen des Verbalnomens des Konditionalis. Solche Verbalnomina bezeichnen den Zustand, gleichgültig ob sie von intransitiven oder transitiven Verben stammen. Die Possessivsuffixe drücken das Subjekt des Geschehens aus. Die Übersetzungen deuten die fraglichen Formen oft durch den Gebrauch von Zeitwörtern mit Präsens- und Frequentativbedeutung an, obwohl diese Verbalnomina kein Tempus bezeichnen und

wort $\dot{p} i, p i$ 'Nacht' auch ein "Diminutivum" $\mathrm{O}$ ṕikkōtsè (381). Es ist aber sicher, dass in der obigen Angabe bei dem Wort mit der Bedeutung 'Nacht' keine Häufung von diminutiven Suffixen festgestellt werden kann. Zwischen dem Wort $\dot{p} i$ und der Endung des Px. der 3. Sg. in stellen die Kond.-Suffixe ${ }^{*} \dot{s} \varepsilon j$ und die Desid.-Opt.-Suffixe ${ }^{*} k \bar{u}$ und *kar die Verbindung her. Bei Castrén erscheint das Konditionalsuffix ptsi in einem Zitat nicht nur im Prädikat, sondern auch im Lativ des mit dem Suffix *ta gebildeten Verbalnomens: "mue-ptsi-tānda' | hartta mitsiki ( $\sim$ mitsjuki)" "zum Lebensplatz euch | er selbst gibt" (98). Vgl. noch (C) "njā-tsa-dan tārinjin!" "zu meinem Kameraden nur gibl" (303);

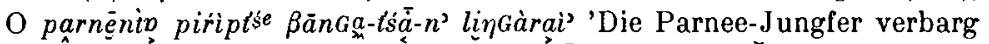

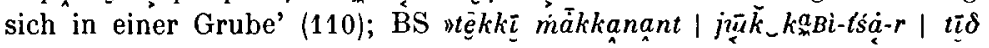
$m i-i s e^{-k} k \bar{u}^{\prime}$ " "Dort in deinem Zelte $\mid$ deine zehn Knechte | verkaufen deine Renntiere" (567). In den Zaubergesăngen, die die Visionen, Bitten, Wünsche des Zauberers ausdrücken, spielt die Betonung der Ir-

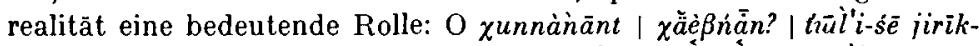

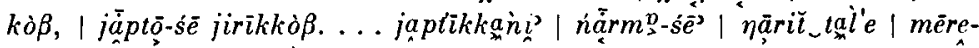

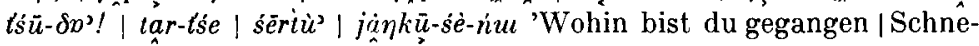
pfe | mein Grossvater, | Gans | mein Grossvater, ... Rötlicher | meines Japtjik-Stammes! | Noch näher herbei | eilet! | Eine solche Angelegenheit | haben sie noch nicht gehabtl' (473). In den Wörtern 'Schnepfe', 'Gans', 'Rötlicher', 'solche' sehen wir dasselbe Suffix śe $\left(<{ }^{*}{ }^{*} \varepsilon j\right)$ wie im

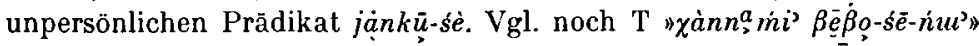

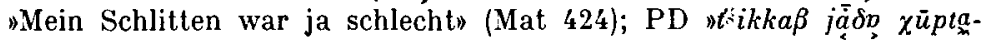
-śe-ńu " "Das Land war aber weit" (ebd. 406) usw, usw. - In den Texten finden sich auch Satzglieder, die das Element ${ }^{*} j \varepsilon$ enthalten. In diesen ist das Element ${ }^{*} j \varepsilon$ das Suffix des Konjunktivs, kein Diminutiv-oder Augmentativ-Suffix, wie die Ubersetzungen es oft durch ein zu-

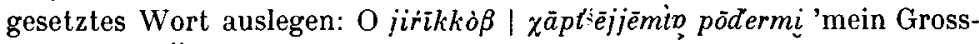
vater, sein Öchslein I schirrte an' $(530$, Zaubergesang); (C) ärkkaje tōje 
auch keine frequentative Bedeutung haben. -- Das Suffix $s \bar{o}, s \bar{u}$ wird meistens mit Passivität und Irrealität bezeichnenden Suffixen verbunden: BS mań $j \ddot{a} r-s \bar{u}-m_{B} i-m$ ' 'Ich

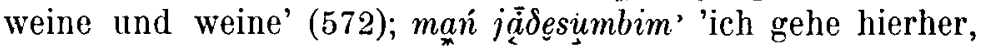

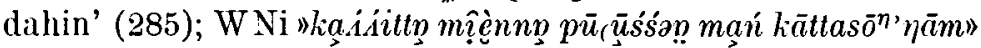
"Ich gehe eine einzelne alte Frau erschlagen" (81); "man

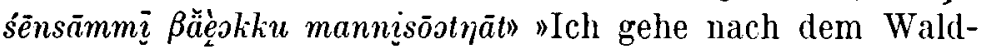

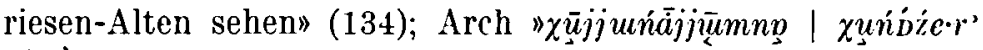

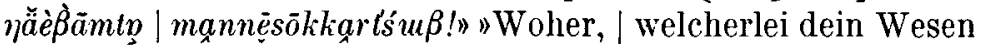
[eig. Kopf] ist, | wenn ich doch schaue!" (182); (C) "hähen dieua ädasun" "Unser Götzenrenntier schirre ab" (304); 0

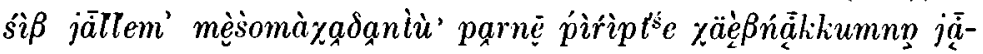

'Es ist ein grosser See' (86). In den "Heiligen Bärenmärchen" (Nr. 51.) begegnen zahlreiche Formen mit dem Suffix $j j \dot{a}: \eta \bar{o} B^{3} \eta_{\bar{a}} k_{k} k_{m} j j \bar{a} \dot{a}$ jaxà '[ein

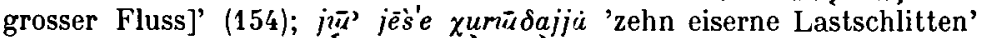

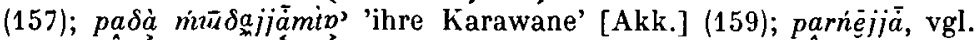

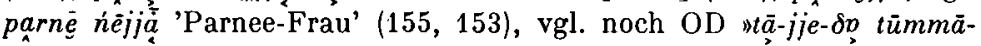
$\delta a{ }_{n} i^{\prime} . . . " W e n n$ ich von dort gekommen bin ..."(72). Auch das Desid.-Opt.-Suffix $k k \bar{o}, k k \bar{u}$ kommt in "determinativem" Sinne vor: U

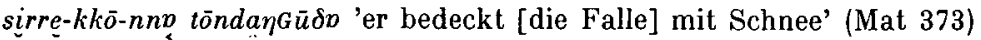
usw. - Was zur dimin. bzw. augm. Deutung dieser Suffixe den Anlass gab, das lāsst sich damit erklären, dass manchmal der Bedeutungsinhalt des Satzganzen und der Grundwörter der entsprechenden Formen bis zu einem gewissen Grade das enthălt, was man hineindeuten konnte:

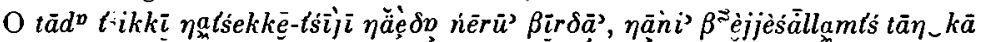
'Dann kratzten die Weiden die Beine jenes armen Kindes, blutlos

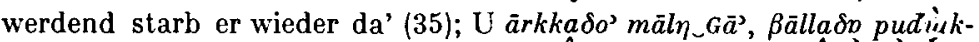

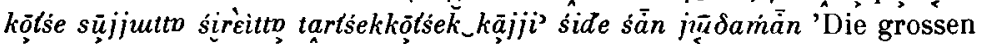
Tiere starben alle, nur von den kleinen Kälbern, den einjährigen Renntierkühen, blieben ungefähr zwanzig nach' (Mat 350); O parnēnt píriptie

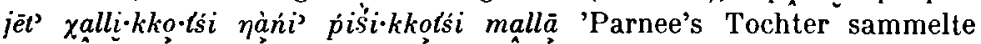

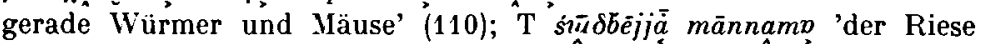
sagte' (93), vgl. sin̄ $\delta b e$ (ebd.), T siū $\delta \cong b e$ 'Riese, Riesenkobold, Waldgeist; sehr gross' (Wb 456; $b e<{ }^{*} p \varepsilon+j$.). Diese Angaben, deren Zahl noch vermehrt werden könnte, bestätigen Ravilas folgende Feststellung: "Die Benennung 'diminutiv' sagt an sich sehr wenig, und dass es sich hier nicht um eine gewöhnliche diminutive Funktion handelt, dürfte ohne weiteres klar sein. Es wăre auch äusserst sonderbar, wenn es tatsächlich Zeiten gegeben hätte, da für den Ausdruck der 'Kleinheit' eine verblüffende Menge verschiedener Suffixe notwendig gewesen wäre. Nach meiner Auffassung ist die diminutive Bedeutung eine sekundäre, auf die man auf verschiedenen Wegen gelangte..." (Virittäjä 1945. S. 151). - Vgl. noch S. 178, 213, 218. 
desumpi 'nach sieben Tagen wandert die Parnee-Jungfer

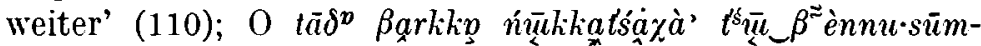
maxà' 'Darauf zogen sich [Du.] die Bärenjungen aufrecht' (151). Das Suffix sō erscheint auch mit anderen Suffixen verbunden in Formen mit Kasussuffixen: (C) tjiki wadida muesomahadanda ... 'Nachdem er diese Worte gesagt hatte...'

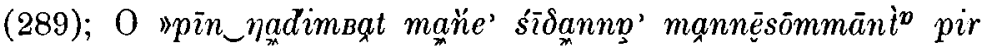
$\beta \overline{a l l a k k a j \delta}$..." "Wenn du hinausgehst, siehst du uns nicht mehr. ... (149) usw. - Für Verbalnomina auf tsü liegen

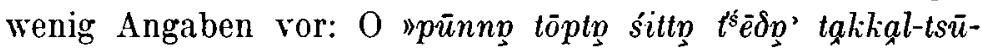
$\delta$-m" "Wenn er später kommt, verstecke ich dich jetzt" (151); (C) "nū $(v)$ un dain pōdertsun, | teän jīnjen moaptsun!" "mein Himmelsrenntier schirre an, I am Lenkriemen meines Renn-

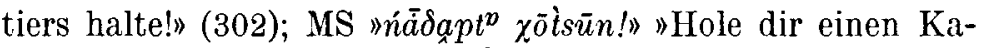

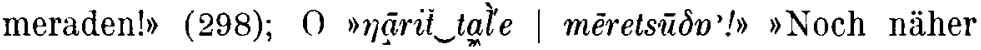

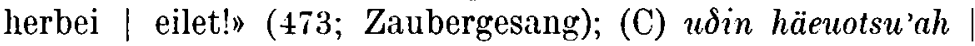
uঠin jidimsu'ah 'meine Hände werden steif, | an meine Hände kommen Blasen' (299).

F ü r V e r ba l n o m i n a a u f psū, ptsū haben wir mehr Angaben: (C) "jileurvāban | harna döptsum» "Wenn ich lebe,

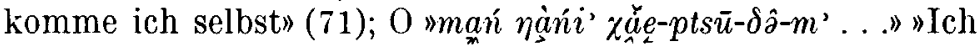

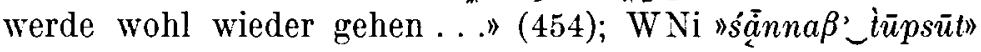
"Ich komme bisweilen" (139); (C) "jilèbnan | sit'eh hōptsum"

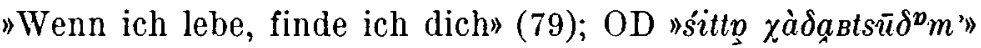

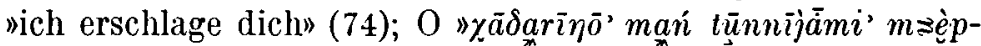
tsūn" "Gewiss nimmst du wohl meine [grosse] Büchse» (50); (C) "hūnjāna | hanod huran, | punjān gäptsjuni'" "Morgen | schnüre deine Schlitten, | zurück wir [Du.] gehen!» (224); MB (BS)

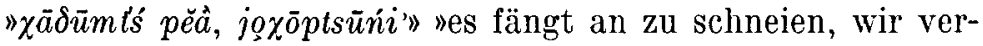

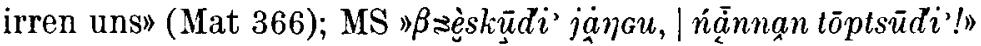
"Ihr [Du.] habt keinen Mann, komm zu mir!" (263); O " $\chi_{\alpha} \delta a-$

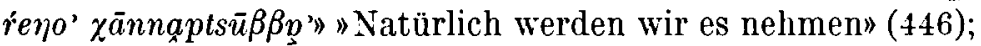

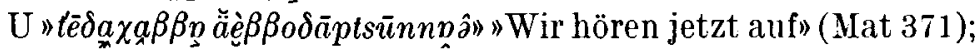

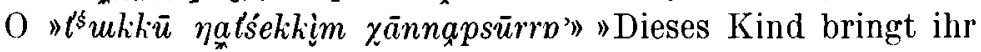
sicherlich fort" (454; vgl. 450). - Als Prädikat in der 3. Sg. erscheint der Verbalnomenstamm: 0 " $\chi \bar{a} \delta^{e} \dot{r} e \eta o^{\prime}$ pador $\chi \bar{a} \bar{j} j \bar{e} \grave{l}$

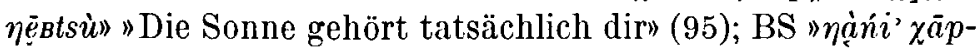
tsū_di" »[er] stirbt noch dort" (283; vgl. Mat 456), MS "pudè 


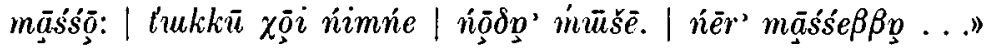
"Er sagte: | Auf diesem Landrücken | fahret nicht. | Sagte doch eben ... (259). Am Ende der Formen der 3. Sg. und 3. Pl. ersetzt der Kehlkopfverschluss das abgeschliffene Posses-

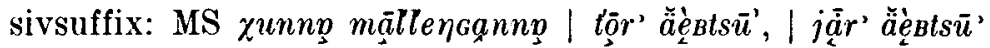
'irgendwann | kommt Geschrei, | kommt Weinen' (254), "āni śu'u| $\chi \bar{a} \delta a p t s \bar{u}$ '» »auch mich erschlagen sie» (301). Selten erscheint das Px. der 3. Pl. doch: (C) "ninjānda dōsa'h, | hādaptsudoh" "wenn er nicht acht gibt, | erschlagen sie sie" (243), vgl. Oks (MS) tī mäèsssin_dāpssūiō' 'vielleicht geben sie Renntiere' (Wb 476).

Verba I nomina a uf setté, sotu. In den tundrajurakischen Texten Lehtisalos entspricht dem waldjurakischen Verbalnomen auf sotu, dessen bedeutende Funktion als Prädikat weiter oben (S. 175) erwähnt wurde, ein Verbalnomen auf setti ', sti $\bar{\imath}^{\prime}$. Vereinzelte Angaben besitzen wir auch für die

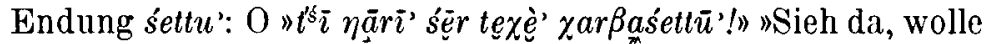

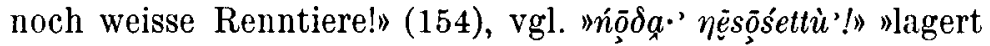

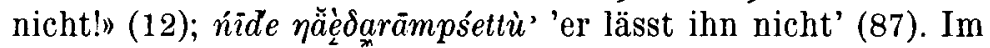
Wortinnern erscheint das Suffix als -śetta-: O $t \bar{a} \delta^{n}$ śà̀nty

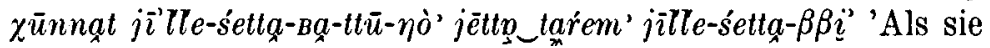
dann einige Zeit lebten, sie lebten gerade so' $(32 ; \mathrm{vgl} .11,17)$;

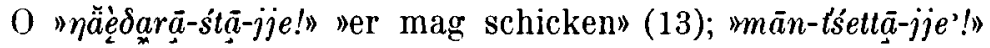
"sie mögen sagen" (9). In Castréns Texten findet sich das Suffix meistens mit $h$ im Auslaut, so z.B. in den Formen der 3. Sg. des Verbs 'fortgehen': häe-setih $\sim$ häe-sitih (187), häe-stih (221), vgl. häe-sete (102), häe-site (219). Beachtenswert sind die beiden folgenden Angaben: müusē-site 'sie fahren mit ihren Karawanen' (244); meätan tjūri-sjetieh ( tjūri-sjeț̄'eh) '[die drei Frauen ... gehen immer in das Zelt' (282). Auf Grund der Angaben Castréns kann festgestellt werden, dass sich das Suffix setti ' $\sim$ sotu auf eine Verbindung ${ }^{*}{ }^{*} \varepsilon j+t \varepsilon+{ }^{*} j \varepsilon$ bzw. $\beta$ zurückführen lässt. Verbalnomina mit derartigen Suffixen haben nominalen und passiven Sinn, sie begegnen in der Sprache der Gesänge und der Sagen, oft in Zitaten, und nehmen die Possessivsuffixe an sich: MS āmd $\bar{c}$ -

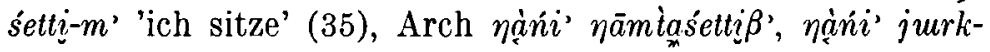


kasetti $\beta$ ' 'wieder setze ich mich, wieder stehe ich auf' (436);

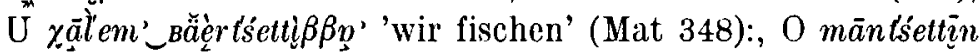

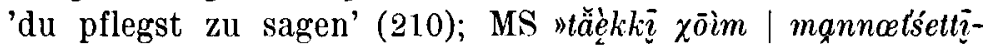
$r \bar{u} \beta$ "? "Jenen Landrücken | siehst du [ihn]"? (277; Frageform); "śênne mānśț̇op'" "kürzlich pflegtet ihr zu sagen" (306); O taŕem' jēttp jilleśsttī ¿i' 'so gerade lebten sie' (31). An die Formen der 3. Person von transitiven Verben werden Possessivsuffixe angehängt: (C) puda hada-sitte-da, | idse-sitte-da 'er' tötet ihn, | hängt ihn' (244); vgl. Arch pōde rt'setț̄ $\delta p$ 'sie schirrt es an' (435); (C) matorsetidi' 'sie [Du.] hackten [ihn] in Stücke' (187); matorsitido id. (Pl.) (ebd.). .- In den deutschen Übersetzungen dieser Verbalnomina fallen die als Erklärung dienenden Wörter 'stets', 'immer', 'immer wieder', 'pflegt' usw. auf. Das Suffix hat aber keine kontinuative Funktion. Zu einer solchen Deutung haben Sätze Anlass gegeben, in denen das Prädikat eine Zeit- oder Umstandsbestimmung hat: WNi pōn jiśáisotu '[er] lebt lange' (132); O tarem' pōn' jit-

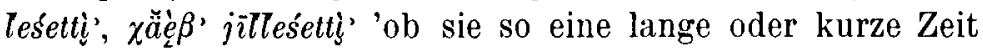
lebten' (6; stehende Wendung) usw. usw. Das Verbalnomen drückt also keine Zeitstufe, also auch keine Gegenwart aus:

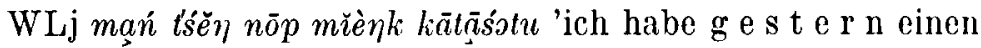
Bären erlegt' (Wb 176).

\section{Das sog. "Präteritum"}

Die "zweite Zeit» drückt nach der Grammatik immer das Präteritum aus, sein Zeichen ist das nach den Personalsuffixen auftretende Element $s i, s, c, t, d$ (Gr. $\$ 475,477)$. Bereits Györke hat darauf aufmerksam gemacht, dass solche Präteritumformen in den Texten ziemlich selten vorkommen (NyK L, 89). Dies hat zur Ursache, dass diese Formen nur in Zitaten, in Reden der Personen, in der Sprache der Gesänge und der Sagen gebraucht werden. Der von Castrén aufgezeichnete Gesang Nr. 9 beginnt mit den Worten: sjuddubaepts mah: 'Das Heldenlied sagt:' (223). Und im Gesang Nr. 4 bemerkte der Sänger: (siudubaepts(n) hanada, | njabi häjih.) | '(Das Heldenlied berichtet [jetzt] von 
ihm, | der andere [Bruder] blieb.)' (143). Der Sänger betont also, dass er eine Geschichte erzählt, die er von anderen gehört hat. In einer heiligen Sage lesen wir die folgende Frage

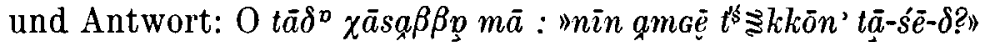

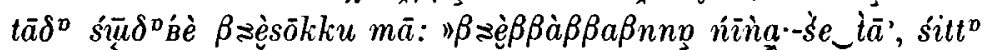

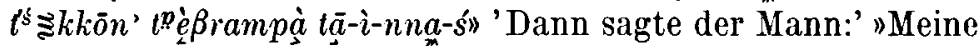
Brüder, warum hast du sie hierher gebracht?" 'Darauf sagte der Riesen-Alte': "Im Bösen habe ich sie ja nicht gebracht, um dich hierher zu bringen, brachte ich sie." (48) Wenn wir

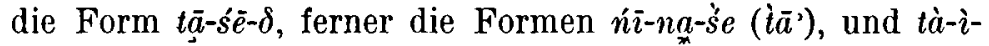
$n n a-s$ nebeneinander stellen, so fällt gleich die Identität der $s$-Elemente des Konditionalis und der Präteritumformen auf. Für Fragen und Antworten dieses Typs liessen sich noch viele Beispiele anführen (vgl. z.B. O 162, PD 122). In Castréns Texten begegnet das Element $s i$ in den Präteritumformen am häufigsten: »aunān | atseki äebnān | njaß̄̄ je waekana | $h \bar{a}-n \bar{a}-m-s i, \mid$ atseäbnān | seädan gara jāna $|h \bar{a}-n \bar{a}-d a-m-s i|$ nienjumde | wäto-r-ngā-si» "Einst als ich jung war, | an das Ende eines anderen Landes | ging ich, | als ich jung war, | ins Land der Hügelkrümmung | ging ich, | seine Tochter

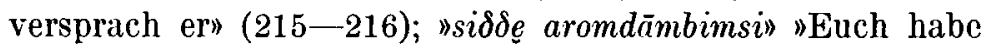
ich erzogen" (256). In Lehtisalos Texten sehen wir in den

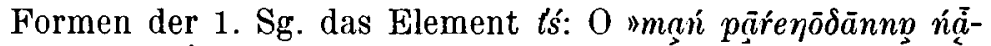

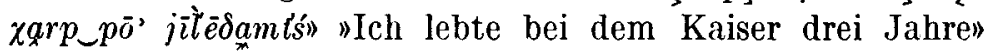
(39); T "śêrttā $\delta a m t ' s "$ "I Ich habe gemacht" (Mat 421). In einigen Angaben erscheint die vollständigere Form des Suffixes auch bei Lehtisalo: 0 "śéńne mājamśe-nu "Ich sagte doch unlängst" (88); vgl. "ńêr' māmóze-ńu'" "Ich sagte ja jüngst" (312); MS "śēñne' māmźīinu'» "Früher doch sagte ich" (264).

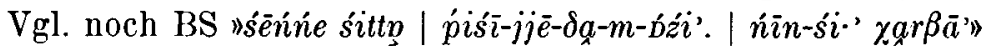
"Kürzlich habe ich dich | nicht gelassen. | Du wolltest nicht"

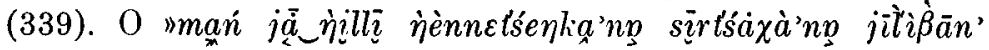

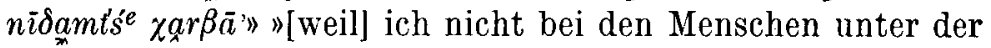
Erde, bei den Tschuden weilen wollte» (156). - In den Formen der 2. Sg. erscheint das Possessivsuffix vor dem ś-Element in der vollständigeren Form na: (C) "nier pūnjāna | mua-na-s» "Soeben auf der Hinterseite du warst" (259; vgl. 232); Arch lakkompọi jạdjernānnaś" "Eine kurze Zeit gingst du" (429); O 
"śêninie' siß $\chi \bar{a} n n a m^{\prime} \chi \bar{a} \delta \bar{a} n n a s$ " "Kürzlich tötetest du sieben Blutopfer" (19). Die Endung hat ebenfalls in den folgenden Belegen die vollständigere Form: MS "māmmo-nō-ra-śe-nu': | $m \bar{u} \delta^{n} \_\delta \bar{o} " ~ " \mathrm{Du}$ sollst aber gesagt haben: | Eine Karawane

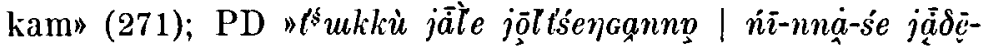
layk $k \bar{u}$ '" "An diesem Tage | wärest du lieber nicht spazieren gegangen» (Mat 398). Die vollständigere Form begegnet auch

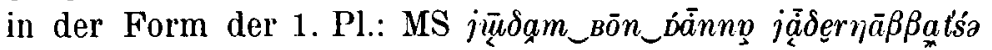
'Vor zehn Jahren gingen wir' (298). - Vgl. noch OD "ńä $\ddot{a} m$ -

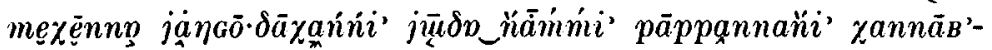

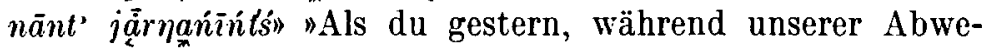
senheit unsere zehn Kameraden, unsere jüngeren Schwestern fortführtest, weinten wir [Du.]" (109). ${ }^{1}$

Die auf die 1. und 2. Person bezüglichen Angaben, in denen die Endung in der vollständigeren Form mit Vokal erscheint, machen darauf aufmerksam, dass das Präteritumzeichen und das Suffix des Konditionalis zusammengehören. Die Formen der 3.Sg. ohne Possessivsuffix, die der Form nach nicht vom Verbalnomen des Konditionalis getrennt werden können und die sich auch in der Bedeutung - wenn wir uns von der Auslegung der Übersetzungen unabhängig machen -.- decken, machen diese Annahme in vollem Masse wahrscheinlich. In den folgenden Angaben sehen wir das Verbalnomen des Verbs

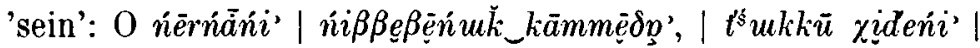

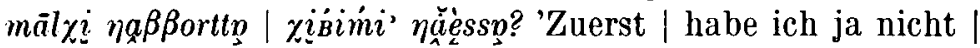
begriffen, | wer war | der Esser, | am Ende dieses meines

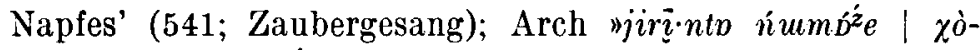

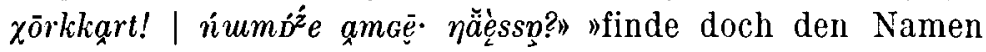
deines Grossvaters! | Wie mag sein Name sein?" (184). In vielen Angaben erkennen wir das mit dem Suffix ${ }^{*} s^{\prime} a+j .(i)$ $\sim{ }^{*}{ }^{\prime} \varepsilon+j$. (i) gebildete Verbalnomen des Konditionalis: $\mathrm{O}$

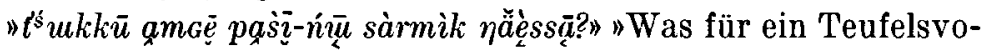
gel war dies?" (34); BS mńinnp jậGusā-m?" "Haben wir Angehörige gehabt?" (282). Bei Castrén hat die Endung si in einer Angabe die Variante sah (< $\left.{ }^{*} s a j\right)$ waäsako ham mäsı? ( ham-

\footnotetext{
${ }^{1}$ Castrén bemerkte, dass die Endungen in den Formen des Duals vor dem Zeichen des "Praeteritums" in ihrer vollen Form erscheinen: 1. $\dot{n}^{\prime}\left(\operatorname{nin}^{\prime}\right)$; 2. $t i^{\prime}, d i^{\prime}(t i n, d i n)$; 3. $h a^{\prime}, g^{\prime}, k^{\prime}($ han, gan, kan)(Gr. S. 225).
} 
ma-sah), | tarem muedo' 'was der Alte sagte, so taten sie' (166). In den folgenden Zeilen eines Gesanges sehen wir Verbalnomina auf $s$, si, tsi: jinjan mam'ah:| härundānau | jängou-n-gā-si-nju'uh, | njār njinjekau | njār tasinjī | nisean dāti nju'uh |jiles häji-tsi,| opoj njēveau |saeusi nieveau|jiles häji-s; | tjet ninjikau | āna hävui-tsi'Ich dachte: | einen Totkranken | hatte ich doch nicht, | meine drei älteren Brüder | die drei Tasinjii, | die Söhne des jüngeren Weibes meines Vaters | blieben lebend [zurück]' (281). Im Satz 'hatte ich doch nicht' erscheinen die Irrealität bezeichnenden Suffixe ${ }^{*} p .+n .+$ $k a+j$ vor der Endung si beim unpersönlichen Zeitwort mit der Bedeutung 'es gibt nicht, ist nicht vorhanden': ein Beweis dafür, dass es sich nicht um ein Präteritum, sondern um irreale Geschehnisse handelt. In der Negation hat die Konditionalform des verneinenden Verbs mehrere Varianten (manchmal mit der Bedeutung des Konditionalis auch in der'

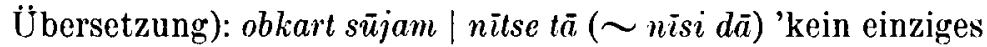
Renntierkalb gaben sie' (87); vgl. njise daeu 'ohne zu erreichen' (217). Auch in Lehtisalos Texten erscheint das Verbalnomen des Konditionalis in seiner vollständigeren Form: BS jinnąin

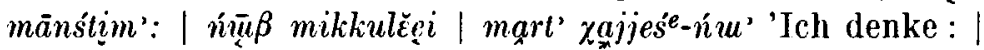
mein Sohn Nikolaus | hatte sich ja in die Stadt begeben' (570);

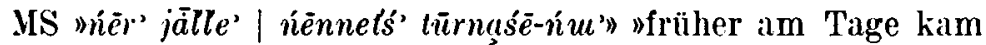

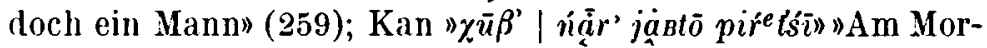
gen | drei Gänse immer kocht er” (219). -- Es gibt nur vereinzelte Angaben für solche Formen der $3 . \mathrm{Sg}$. und $3 . \mathrm{Pl}$,

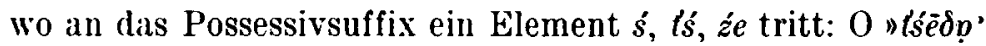

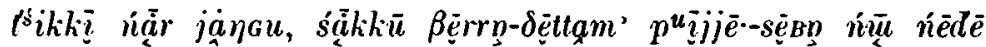
$\chi \bar{a} \delta \bar{a}-\delta a s "$ "Jetzt ist dieser dein Kamerad nicht, die Tochter von Trockennase, sein Weib, hat Gänseknöchelhaar-Wirt

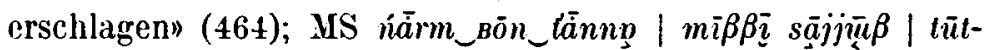

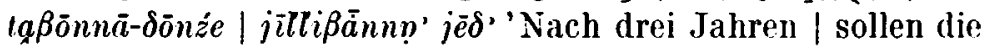
gesammelten Krieger | beabsichtigt haben zu kommen | unserer Renntiere wegen' (298).

In den Texten erregen unsere Aufmerksamkeit solche mit dem Suffix $\eta k \bar{u}$ gebildeten Verbalnomina, die - wie weiter unten erörtert werden soll - kein Geschehen in der Vergangenheit bedeuten können und bei denen - nach den Pos- 
sessivsuffixen - doch dieselben Endungen zu sehen sind wie bei den sog. Präteritumformen. Aus einem Zaubergesang zitiere ich die folgenden Zeilen: $m \approx \grave{e} \delta$ arkkānng $\left|\operatorname{tańn}_{x} \bar{\eta} \eta \hat{u}-s^{t}\right|$

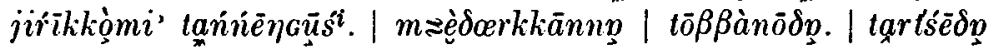

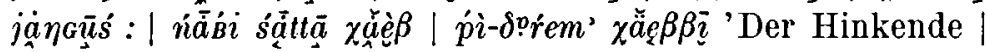
sollte sein, | mein Grossvater | sollte [hier] sein. | Den Hinkenden | hörte man kommen. | Derartiges hat er nicht gehabt: | die eine Seite seines Gesichtes | ist wie die Nacht geworden' (542). Auch die beiden folgenden Belege sind zu beachten:

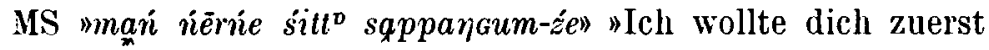

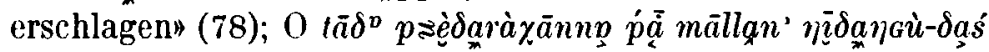
'dann wollte er ihn im Walde an einem Baumwinkel erhängen' (24). Aus dem Jurakischen kann ich noch regelmässige Präteritumformen anführen, die wegen der Grundbedeutung des Verbalnomenstammes kein Geschehen in der Vergangenheit bedeuten können: (C) "tjili paejam | jilhauāpta | njuu mi'idam-si" "Den grossen Stein, | wenn er ihn hebt, | meine Tochter würde ich geben" (292). Der Kehlkopf verschluss in der Form mi idamsi steht für das Suffix *'s des Konditionalis; das desiderative Suffix *ta bedeutet nur ein Geschehen, das in der Zukunft erfolgen mag. -- In einem Gesang aus dem MS-

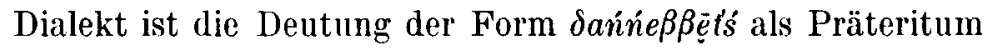
ein Irrtum: einer der beiden Brüder fragte das Mädchen, ob sie einen Bruder, einen Vater, eine Mutter habe, und das Ge-

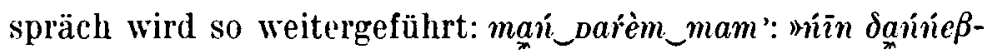

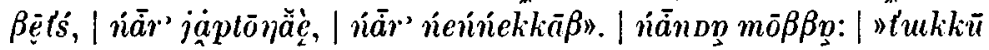

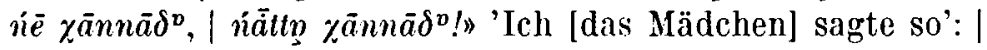
"Ich h a t t e Brüder, | drei Japtongäe, | drei ältere Brüder." | 'Er sagte zu seinem Bruder': | "Bringe dieses Weib, | bringe es zu seinen Brüdern!" (352). Man kann das Mädchen nicht zu solchen Brüdern bringen, die nur gewesen sind; die Form Sanine $\beta \beta \overline{-}-$ ist cin Verbalnomen passiver Bedeutung, es bezeichnet einen Zustand, und das Element t's kann keine Vergangenheit ausdrücken, sondern den Konditionalis: 'ich hätte Brüder' [sie sind aber entfernt von hier]'.

Um diese jurakischen Formen zu verstehen, müssen wir die Formen des selkupischen Futurs (»dritte Zeit») beachten. Bei diesen erscheint nach dem Personalsuffix das Element 
$h e \sim s e \sim s i \sim s$, "welches ohne Zweifel denselben Ursprung hat wie der Charakter des Practeritums» (Gr. § 477). Castrén hat also das Element $h e \sim s e \sim s i \sim s\left[{ }^{*}{ }^{*} \varepsilon(j)\right]$ des Charakters des selkupischen Futurs und das Zeichen $h a \sim s a\left[<{ }^{*}{ }^{\prime} a\right]$ des selkupischen Präteritums für gleichen Ursprung gehalten, das letztere erscheint - ebenso wie das Suffix des jurakischen Konditionalis - vor dem Personalsuffix. Der Charakter des selkupischen Präteritums bzw. Futurs ist von derselben Herkunft wie das Suffix des jurakischen Konditionalis.

Auf Frund der Angaben aus den Texten gewann ich die Überzeugung, dass das Jurakische keine "zweite Zeit" mit der Bedeutung des Präteritums hat. Nach den Possessivsuffixen der als Präteritum gedeuteten Formen erscheint das Suffix ${ }^{*} \boldsymbol{s} a$ bzw. ${ }^{*} \boldsymbol{s} a j \sim{ }^{*} \boldsymbol{s} \varepsilon j$ des Konditionalis, und diese Formen haben auch ihrer Bedeutung nach konditional-potentialen Sinn.

Es soll noch die Frage beantwortet werden, warum das Suffix des Konditionalis in den erwähnten Formen nach den Possessivsuffixen erscheint. Die Antwort kann auf Grund der Erkenntnisse aus den kamassischen Konjunktivformen gegeben werden. Diese Forınen entstehen nach Castréns Grammatik (s. \$552 und die Paradigmen) in der Weise, dass die personalsuffigierten Formen des mit dem Suffix $n a$, nä srebildeten Verbalnomens mit dem Verbalnomen des Konditionalis des Verbs 'sein' $i z \ddot{a}$ verbunden sind. In solchen Fügrungen ist $i z \ddot{a}$ ein selbständiges Glied, z.B. (nere- 'erschrek-

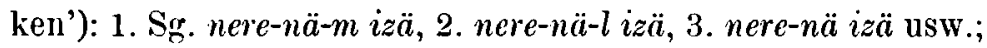
(phargar- 'schnitzen'): 1. Sg. pharga-na-m izä, 2. phargā-na-l izü, 3. pharga-na-t izä usw. In der kamassischen Grammatik von A. J. Joki, die auf Grund der Aufzeichnungen von Kai Donner geschrieben wurde, sehen wir aber, dass das Verbalnomen $i z \ddot{a}$ seine Selbständigkeit eingebüsst hat und zu einer mit den Personalsuffixen der Konjunktivformen verbundenen Endung geworden ist ( $\mathrm{D}-\mathrm{J} 169--170)$. Dieser Prozess erfolgte auch im Jurakischen. Das Verbalnomen des Konditionalis vom Verb 'sein', gebildet mit dem Suffix *sa, *ś, *saj, *scj (das ebensowenig in den Paradigmen des Verbs 'sein' erscheint wie das kamassische $i z i \ddot{)}$, wurde ursprünglich als selb- 
ständiges Glied mit dem personalsuffigierten Verbalnomen verknüpft, ebenso wie das kamassische $i z \ddot{a}$ zur Zeit Castréns. Es wurde dann nach Einbusse seiner Selbständigkeit zu einer Endung ebenso wie in den Formen des kamassischen Konjunktivs, der selkupischen »dritten Zeit» wie auch in den Formen der sog. "zweiten Zeit" des jurakischen Konjunktivs.

"K o n ju n k t v z we i t e Z e i t." In Castréns Konjugationsparadigmen gibt es Formen unter diesem Namen. In den Angaben aus den Texten nehmen die mit den Suffixen $n \bar{\imath}\left(<{ }^{*} n \varepsilon+j\right.$.) bzw. $j \bar{\imath}$ gebildeten Verbalnomina Possessivsuffixe an sich und nach den Possessivsuffixen tritt das Suffix des Konditionalis auf. Die Bedeutung solcher jurakischer Formen steht der Bedeutung der finnischen Konditionalformen und den ungarischen mit dem Suffix na, ne gebildeten Formen nahe. Das Suffix $i i$ bzw. $j \bar{i}$ ist manchmal mit den Suffixen ${ }^{*} p a$ und ${ }^{*} t a$ verbunden. Die Zahl der Angaben ist ziemlich gering. Die fraglichen Formen kommen ausnahmslos in Zitaten, in der gesprochenen oder gedachten Rede der Personen vor: (C) jūb jundar-ni-m-si, | wanji sierij èngü? 'Gern würde ich es erkunden, [aber] es geht mich nichts an' (7; stehende Wendung im Gesang), vgl. b. jundar-ji-da-m-si (20) c. junarā-wa-sj (28); MS têt sệrrakkōo | oppò̀imtp $\chi \bar{a} \delta p$-ní-

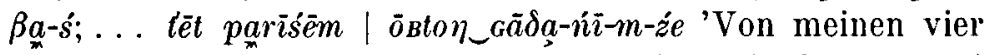
Hellen | würde ich eins töten . . von meinen vier Schwarzen | würde ich eins töten' (302); MS nōbkannn jille-ní- $\beta \beta a-t_{x}$ 'sen "Wir

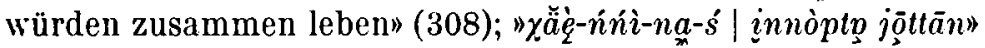
"Wenn du gehen würdest, | verlierst du deinen Bogen" (276); - (C) "huukum beäkutsem(ah) | nipnjanda njam(ah) | hartta vual-ni-s" "Den rom Wasser getriebenen kleinen Baum, | hätte er ihn nicht erfasst, | hätte er selbst gesprochen" (298). Die Variante víné sehen wir in einer Fügung mit dem Verbalno-

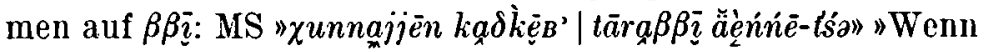
irgendein Schlimmes geschieht, | hätte man sie gebraucht» (253).

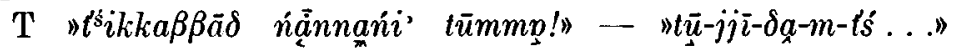
"Komme dann mit mir!" — "Ich käme, [aber] . . ." (Mat 423); "nérrne man $\chi \bar{o} r-j \bar{i}-\beta a$-s" "zuerst würde ich versuchen" (92);

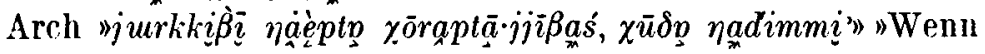


er aufstehen würde, würde ich fragen, woher er kam» [eig. sichtbar wurde] (437). O "jīis' śêngajińniñt'ś" "Recht gut wür-

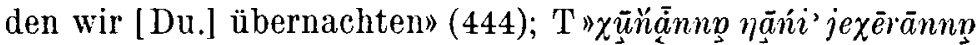

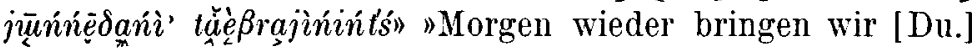

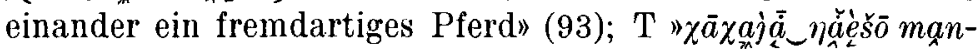
néjinnat's" "Wir könnten sie uns ansehen, wenn wir in der Nähe lagern" (Mat 418). Es gibt auch einige Angaben für die

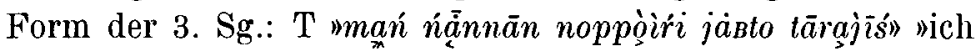
würde nur eine Gans brauchen" (Mat 419). - Mit der Form näèjjīs des Verbs 'sein' entstehen Konstruktionen mit Ver-

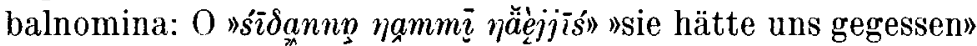

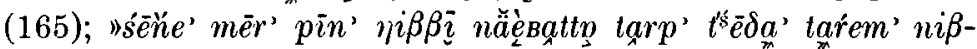
$\beta \bar{e} n$ njăèjiss majjentōr'" "Wenn du damals nicht bald hinausgetreten wärest [aus dem Zelt], hättest du jetzt nicht so gelitten" (156, das Px. der 2. Sg. tritt an die mit $\beta \beta \bar{\imath}$ gebildete Form des verneinenden Verbs).

Das Gerundium (der Infinitiv) auf $s$, t's

Castrén hat festgestellt, dass im Samojedischen ---- mit Ausnahme des Selkupischen und des Kamassischen - "der Infinitiv teils durch Nomina verbalia, teils durch das Gerundium ausgedrückt» wird. Er hat ferner festgestellt, dass es im Jurakischen drei Arten von Gerundien gibt: das Gerundium der vokalisch auslautenden Stämme, welches sich mit der Form der $3 . \mathrm{Sg}$. des Indikativs (1. Art) deckt; das $b$-Gerundium und das Gerundium, dessen Charakter $s, z,(t, d)$ lautet (Gr. § 492). ${ }^{1}$

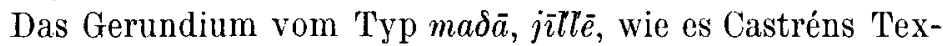
ten nur vereinzelt vorkommt, kann mit dem Verbalnomen auf ${ }^{*} j \varepsilon(i)$ gleichgesetzt werden, das $b$-Gerundium aber - wie

${ }^{1}$ Lehtisalo meint: "Möglicherweise ist das juraksamojedische Infinitivsuffix als Verbalnomen auf *'s aufzufassen» (AblSuff 200); Björn Collinder: "There was no infinitive in PU, strictly speaking. Instead, case forms of various verb substantives were used" (CompGr. $\$ 769$ ); Péter Hajdú: "Das Lativsuffix $s$ ist m.E. mit der jur. Infinitivendung $-\dot{s} \sim-\frac{\dot{z}}{2},-t \dot{s}$ gleichen Ursprungs》) (vgl. CIFU 269-271). 
weiter oben erörtert (S. 175) - - mit dem Verbalnomen auf * $p a$. Das Gerundium mit einem ś-Element findet sich in Castréns Texten am häufigsten mit der Endung si, es gibt aber auch Belege mit den Endungen $s, t s e, t s i, t s, t j e, t j i$. In Lehtisalos Texten begegnen uns im allgemeinen Formen mit der Endung $s, t s$, aber in vereinzelten Angaben fallen vollständigere, auch cinen Vokal enthaltende Endungen auf. Wir wollen einige Angaben ins Auge fassen; z.B. 'sein': (C) »side jā $\eta \bar{a} r k k a$ äesi $\mid \overline{o b t a n}$ taeruini'eh" "Da wir [Du.] zwei Grosse des Landes seiend, I

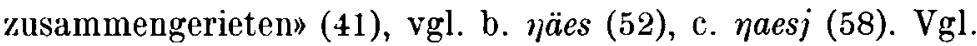

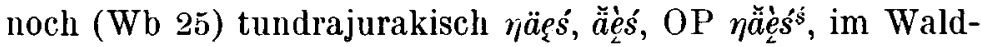

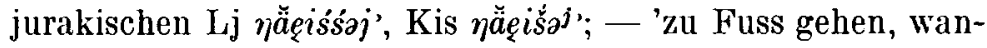

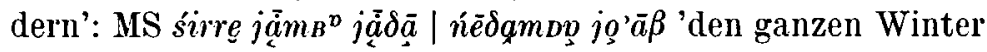
wandernd, | verlor ich seine Schlittenspuren' (351); (C) jädas haijeä 'er begab sich zu Fuss gehend' (117); Kan namyẹejopt | piūriān jādaś?" "Was suchst du zu Fusse gehend?" (239); 0

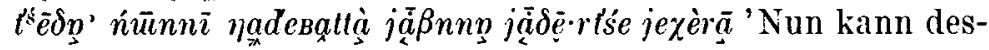
halb der Polartaucher nicht auf dem Lande gehen' (36); -. 'sagen' (in einer stehenden Wendung 'sagend trug vor'): Purŕre-Fl. māñtśe (475), vgl. māñśse (501); O māñtśe (29); Arch māñoźe (182), māntśe (185); - 'anblicken, anschauen': (C) taritse manatse 'beim Anblick dessen' (198); OD »tū jällem' mannēt'ś_`" "beim Anblick des Feuerscheines" (192); -- 'essen': (C) amsi jāmioido '[die andere Hälfte] konnten sie nicht essen' (180); "halmear amzih | ouortse armam" "Totenfleisch | essend wuchs ich heran" (9), vgl. b. ouorts? (21), c. auwartji (29); BS

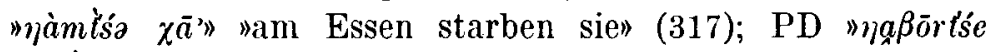
pěà $\grave{\beta}$ " "sie hat begonnen ... zu essen" (108, vgl. 60, OD 73);

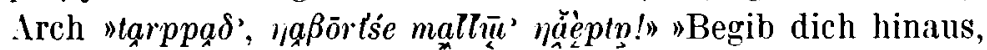
wenn du mit dem Essen aufgehört hast" (426).

Gerundiumformen von Verben (Verbalnomina) passiven Sinnes: (C) nūm paeusemsi... 'Mit Einbruch des Abends'

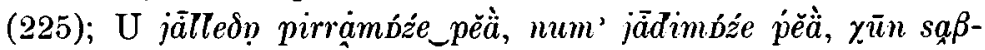
Borkika $a s^{\prime}$ 'Die Sonne fing an hochzusteigen, das Wetter fing an heiss zu werden, am Morgen war es recht gut gewesen' (Mat 359); (C) handa nimnje ämdios | njamāda 'in seinem Schlitten | sitzend packte ihn' (75); tjikị jāna | üäsos(i) beäda 'an dieser Stelle | begann er ein Lager aufzuschlagen'(217), 
vgl. U "êssō dàrạà" "man muss ein Lager aufschlagen" (Mat 358). Aufschlussreich ist eine Angabe aus Castréns Texten:

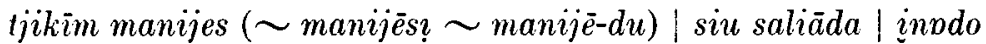
minjeh, | jīnilįd 'Während sie das sahen, | von sieben hohen Halbinseln | ihre Bogen sie tragen, | sie begannen zu schiessen' (97). In der Form manijēedu ist der Stamm ein Verbalnomen auf $* j e$, der mit dem Px. der 3 . Pl. versehen ist. Diese Nominalform beweist, dass zwischen den Bedeutungen der Varianten kein wesentlicher Unterschied besteht; dies bestätigen

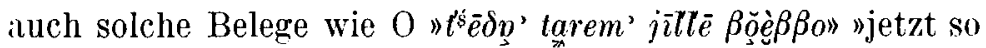
zu leben [ $=$ so ein Leben] ist schlecht" (6).

Verrleichen wir die Endungen der Verbalnomenstämme des Konditionalis (und die der azweiten Zeit») mit den Endungen des Gerundiums, so fällt die Gleichheit der Form auf. Wenn wir in Betracht ziehen, dass das Gerundium in Zitaten, in der Rede der Personen, in der Sprache der Gesänge und der Sagen, sowie in Erzählungen hypothetischer Geschehnisse vorkommt, so können wir behaupten, dass das Gerundium iluf $s, t ' s$ usw. der gleichen Herkunft ist wie der Verbalnomenstamm des Konditionalis. Es gibt Beispiele, wo uns das Gerundium und das Prädikat in völlig gleicher Form begegnen: (C) siu jiri | tanja paerngādi'eh, | madasi beäsi 'sieben Monate | da schiessen sie, | zu durchschneiden begannen sie' (203):

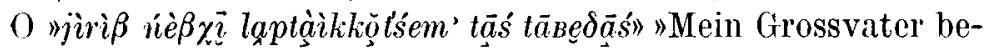
fahl die alte Kiste zu geben" (51) usw.

Um auf die waldjurakischen Formen des Verbs 'sein' $\mathrm{Lj}$

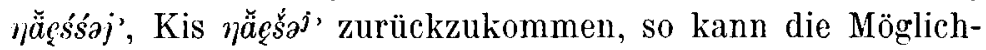
keit erwogen werden, dass der Kehlkopfverschluss für ein abgeschliffenes Element ${ }^{*} t$. steht. Diese Annahme lenkt unsere Aufmerksamkeit auf die Endungen des kamassischen Infinitivs: set, sit, st, zet, zit usw.; $z^{2} t$, žit, šit (D-J § 64). Das kamassische $\dot{z}, \check{z}, \check{z}$ in der Endung des Infinitivs dürfte ein entsprechender sekundärer Laut sein, wie der Laut $s$, t's des jurakischen Konditionalis und Gerundiums. Ist die Annahme richtig, so lässt sich die waldjurakische Endung s'śj', $\grave{s} \partial^{j}$, und die Endung des kamassischen Infinitivs auf ein zusammengesetztes Suffix ${ }^{*} \varepsilon \dot{\varepsilon}+j+t$. zurückführen. Es ist nicht uninteressant, dass bei den kamassischen vokalisch aus- 
lautenden Stämmen ein Element $i, i$ vor der Infinitivendung auftreten kann (ebd.) und dass es auch im Kamassischen ein Gerundium mit dem Suffix $i$ gibt. Dieses Gerundium "bei den meistens transitiven Zeitwörtern bezeichnet, auf welche Weise die Handlung geschieht (z.B. amnai tebaktorla'baja' 'sitzend sprechen sie')" usw. (ebd. §68).

\section{Die Gerundien auf Bans und mans}

Nach Castrén ist das "Supinum" auf wanź, manź, dats eine Ableitung vom Dativ des Verbalnomens auf wa, ma darstellt, "eine sehr seltene Erscheinung", nur im Jurakischen vorhanden (Gr. § 472). Die Texte zeugen davon, dass der Gebrauch der Verbalnomina auf $\beta a n s$, manś nicht so begrenzt ist, wie es sich auf Grund der Grammatik denken liesse. Die fraglichen Formen sind die Lativformen des mit * pa bzw. *ma gebildeten Verbalnomens, an deren Ende das Suffix des Konditionalis erscheint. Es kann als sicher angenommen werden, dass ursprünglich auch diese Formen zweigliedrige Konstruktionen waren: Die Lativformen gingen mit den Konditionalis-Verbalnomen des Verbs 'sein' eine Verbindung ein. Die betreffenden Gerundien kommen in Zitaten, in der Spriche der Gesänge und der Sagen, sowie auch in der Erzählung nicht-konkreter Geschehnisse (Lebensweise, Gewohnheiten) vor. Nach ihrer Grundbedeutung sind es Zustandsbestimmungen, in ihrem Gebrauch aber können gewisse Unterschiede bestehen. Da das jurakische Verbalnomen auf ${ }^{*} p a$ genetisch mit dem ungarischen Verbaladverb $v a, v e$; ván, rén zusammengehört, ist es nicht überraschend, dass die Funktionen des jurakischen Gerundiums auf $\beta a n s$ (und zugleich manś) beachtenswerte Übereinstimmungen mit den Funktionen des ungarischen Verbaladverbs aufweisen. So ist hinsichtlich der jurakischen Gerundien sehr lehrreich, was S. Károly in seinem oben (S. 176) angeführten Werk (Igenévrendszerünk) über die Funktionen des ungarischen Verbaladverbs feststellt. Károly weist nach, dass das Verbaladverb auf ván, vén in der Sprache der ältesten ungarischen Kodexe in Verbindungr 
mit dem Verb 'sein', seltener auch im selbständigen Gebrauch, prädikative Funktion erfüllen kann (S. 160). Auch im Jurakischen können die Gerundien auf $\beta a n s$, manś in prädikativer Stellung den Zustand des Subjekts ausdrücken.

Eine Frau, die den MS (U)-Dialekt sprach, begann die Erzählung der Ereignisse ihres Lebens vor Lehtisalo folgender-

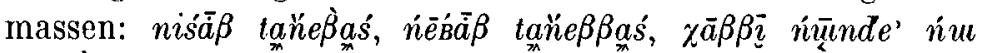

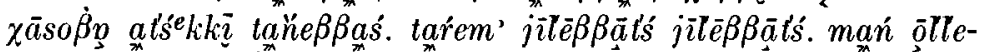

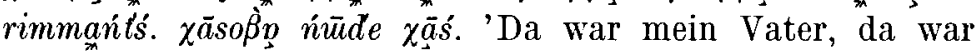
meine Mutter, da war der Sohn ihres verstorbenen Sohnes, ein kleiner Junge. So leben wir, leben wir. Ich war allein, der Junge starb' (Mat 347). Im Laufe der weiteren Erzählung erscheinen neben Prädikaten, die Passivität und Irrealität bezeichnen, auch weitere Prädikate mit dem Element $\dot{s}$, $t^{\prime} \dot{s}$ :

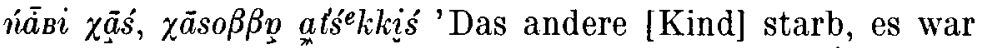
ein Junge' (ebd. 348); tà ńä́nnp tịnn $\chi \bar{a} t t^{\prime}$ 'Im Sommer starben unsere Renntiere, sie starben um den Iljatag herum' (ebd. 350). Bei näherer Betrachtung der ganzen Erzählung können die Formen $\chi \bar{a} s, \chi \bar{a} t^{\prime} s$, at'sekkiś nur als zustandbestimmende Gerundien aufgefasst werden ('gestorben', 'Junge-seiend'), und unter diese fügen sich die zustandbe-

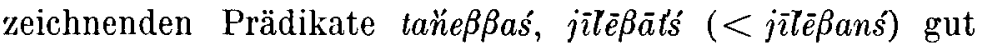
ein, ebenso wie das Gerundium ōllerimañ tśs. Für den prädikativen Gebrauch des Gerundiums mit der Endung $\beta a n s ́$ gibt

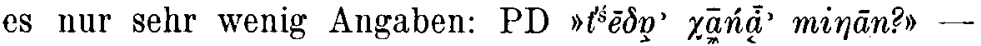

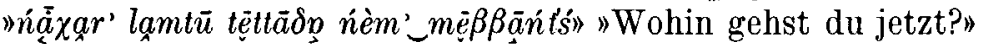
- "Eine junge Frau holen von den drei lamtū Wirten" (Mat 405 ; - es handelt sich hier nicht um eine Zweckbestimmung, sondern um die Bezeichnung des Zustandes); BS jixinniáan

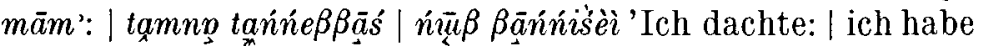
ja auch gehabt $\mid$ meinen Sohn Hans' (572-573). In zwei Kaniner Angaben wird die Endung $\beta a n s ́$ an den konjunktivischen

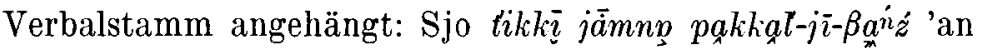

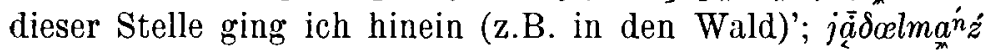

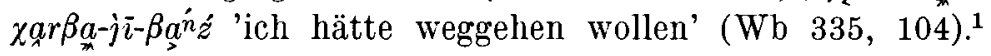

${ }^{1}$ Die beiden Kaniner Angaben werfen ein Licht auf den Weg des Schwundes des Lativsuffixes $n$ und erklären bis zu einem gewissen Grade, warum in Castréns Paradigmen Formen mit der Endung wans, 
Über die figura etymologica -Konstruktionen mit Verbalnomina auf ván, vén schreibt Károly: "In unseren eine eigen ungarische Konstruktion aufweisenden Kodizes wird der Nachdruck des öfteren durch eine figura etymologica ausgedrückt, wobei der Unterschied in der Aktionsqualität zwischen Verbaladverb und Zeitwort betont wird (jövén eljő: veniens veniet)" (a.a.0. 230). Solche Konstruktionen gibt es auch im

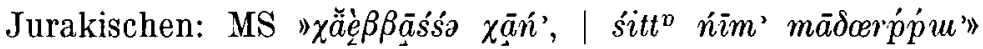

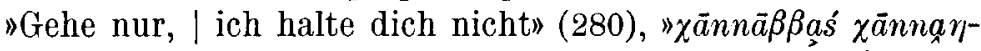

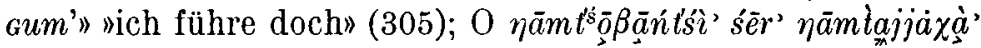
'sie sitzen und sitzen' (10). Beachtenswert ist der Ausdruck (C) "man-si-vān madm» »ich, ich sage» (128). Die Endung -vān wird an den Verbalnomenstamm des Konditionalis angehängt.

Bedeutend mehr Angaben gibt es - meist in stehenden Wendungen - für den adverbialen Gebrauch des Gerundiums auf $\beta$ anś. Die Prädikate der Beispielsätze drücken ein

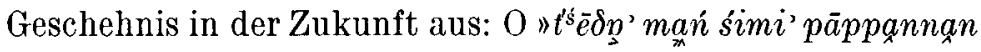
$\chi \bar{a}-\beta \beta$ ann t'se $\chi \bar{a} \bar{j} j \bar{e} n a k k i$ '" "Jetzt haben mich wohl meine jüngeren Brüder verlassen, um zu sterben" (150; vgl. Kan 239, 242);

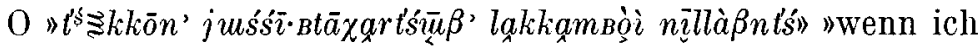
mich mal hier langlegte, um ein Weilchen zu ruhen!» (51; vgl. 153); (C) "manj häengum | han-jie-wan-si | hãntam» "Ich gehe fort, | ich gehe jagen" (117; vgl. O 1, 18, 46, 149, BS 208, 358). In einem Satz erscheint das Gerundium als Zustandsbestim-

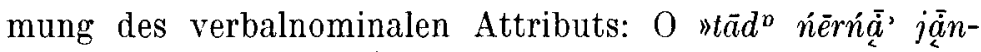

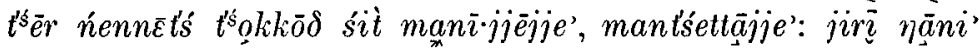

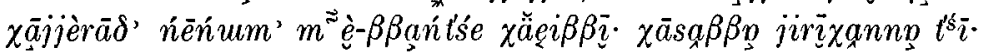
$t \bar{a} \delta \bar{a} \delta p "$ "Von nun an mögen dich die auf der Erde wohnenden Menschen hier sehen, mögen sagen: der Mann, welcher ausging, die Tochter von Sonne und Mond zu nehmen, blieb dort festhalten" ( 9 ; vgl. 8, 457, Mat 405). Auch die folgenden Gerundien mit adverbialer Funktion kommen in Zitaten vor: (C) nieleuansi mingaua' 'ihn verheiraten ... gehen wir' (64); mansara-uansi 'arbeiten [gehe ich]' (122); O ṕintà $\beta n t ' s$ 'Nutz-

wanź (wać) als Formen der II. Zeit 1. Sg. Ind. der sog. reflexiv konjugierten Verba, wie auch als solche der II. Zeit 1. Sg. Konj. angeführt sind: mada-ju-wans, mada-je-wanś usw.; - mada-ji-wanśs -wanź, -want, wać usw. (Gr. S. 391, 394). 


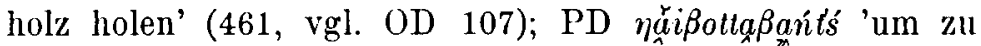

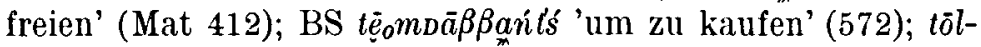

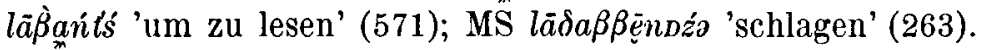
- In einigen Endungen übt das Element $\beta$ eine labialisierende

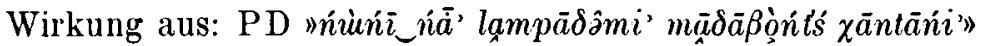
"Wir begeben uns mit meinem Sohne, um Schneeschuhe für

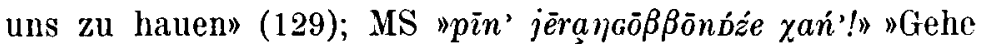
über Nacht die Renntiere hüten!» (75); Kan (Sjo) $m a^{n} z a r a \bar{a} \beta \beta \bar{o}^{\prime} z^{\prime}$

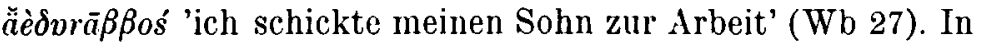
den folgenden Angaben ist das $\beta$-Element der Endung $\beta$ n't's mit dem Vokal des mit * $t a$ gebildeten Verbalnomens verschmol-

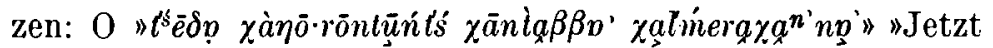
begeben wir uns, um unseren Verstorbenen zu opfern" (466).

Für die prädikative Funktion des Gerundiums auf mans habe ich aus Castréns Texten nur eine Angabe b. "pudara kurkada?" | "manj jiheram, | ani jauna jada-r-n-ga-mansi" "Wie lebt ihr?" "Ich weiss nicht, | in einem anderen Lande bin ich gewandert" (24). (Die mit $r+k a$ gebildete Form des Pronomen $k u$ - ist als Verb konjugiert.) Vgl. c. jadar-mandji (32), a. jadarnga-msi (16). Aus Lehtisalos Texten besitzen wir mehr

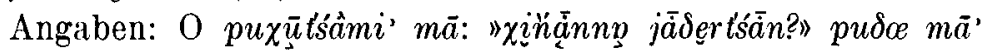

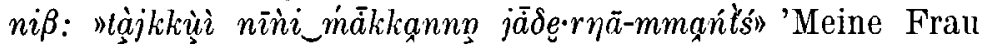
sagte': "Wo warst du?" 'Er sagte so:' "Ich besuchte die Zelte jener Kameraden» (Mat 345. In der hypothetischen Erzäh-

${ }^{1}$ Das obenerwähnte Gerundium vom Typ MS $\chi \bar{a}$ ńjeßrítśa 'um zu jagen' hat auch eine Entsprechung $\chi \bar{a}$ rijeśsa in demselben Dialekt. In Gerundien dieses Typs wird dem Konjunktivstamm das Lativsuffix $n$ und das Suffix des Konditionalis angehängt: MS side jōnnại náa $\delta \dot{o}^{\prime}$

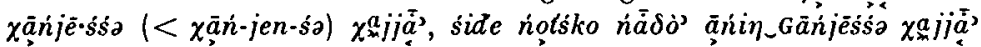
'Die zwei mittleren Brüder gingen auf die Jagd. Die zwei jüngsten Brüder gingen auch jagen' (77, vgl. 276). Derartige Gerundien finden sich

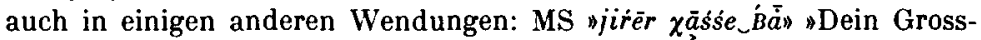

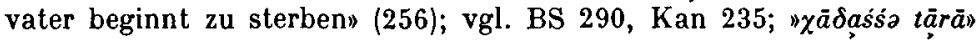
"man muss [ihn] töten" (304); "jilltissśa töB'nan" "wenn ich lebendig kom-

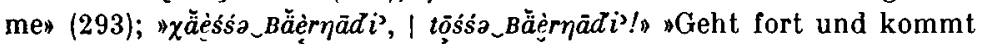
wieder!" (280). Wahrscheinlich können auch die im $\mathrm{Wb}$ angeführten, besonders aus dem OP-Dialekt zitierten Ger.-Inf-Formen mit der En-

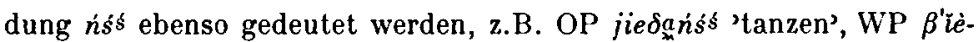

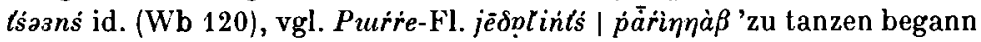
ich' (486). 
lung spricht der Gewährsmann von sich in der 3. Person.) Auch die folgende Angabe stammt aus einem imaginären Ge-

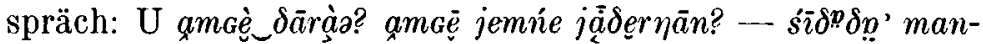
ne'màn't's tōmmañt's. 'Was tut not? Warum kamst du? - Ich kam euch besuchen' (ebd. 352). In einem Rauschlied aus der BS-Mundart sind die folgenden prädikativ gebrauchten Gerun-

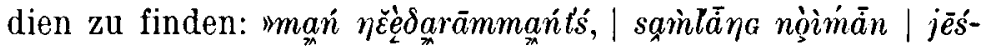

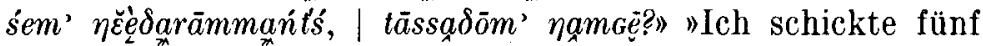
Rubel | Geld schickte ich. | Haben sie gegeben? [Kond. Frageform]» (577). Das Gespräch wird fortgesetzt: "mi t'särū $\beta$ ', namaēe

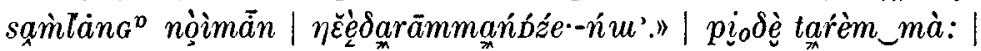

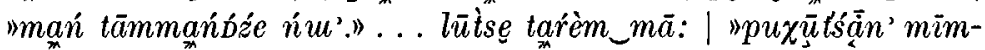
mańt'ś" "Gabst du, was? | Fünf Rubel schickte ich doch." | 'Er sagte so:' | "Ich gab ja» . . . 'Der Russe sagte so:' | "Dem Mütterchen gab ich" (ebd.; vgl. 292, 295). In der Angabe U man ôllerimmañ 's' 'Ich war allein' (Mat 347) ist die Endung man't's mit dem Wort ōlleri 'allein, einzig' (Wb 37) verbunden. Aus der BS- und MS- Mundart können wir noch einige Angaben anführen: BS "śit $\beta \bar{a} \delta \bar{a} m m_{\pi} a n t t^{\prime} "$ "Dich habe ich aufgezogen" (282); vgl. "mall $\bar{e} \beta \bar{a} \delta \dot{a} \beta \beta \bar{e} \hat{\beta} \beta " ~ " I c h$ habe [sie] schon aufge-

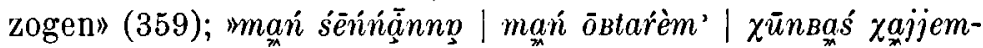
mań $z^{\prime \prime \prime}$ »einst ebenso fliehend | begab ich mich" (330). In einem Zitat aus einem (Helden)lied kommen drei Gerundien vor: mman

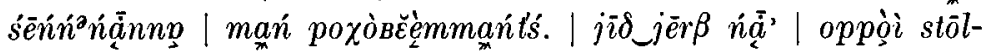

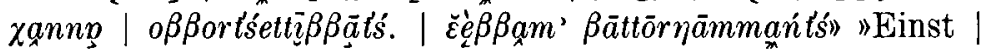
war ich stark. | Mit dem Wassergeist | zusammen am Tische | pflegten wir zu essen. | Den Kopf versprach ich» (291). Ein Mann begann die Erzählung der Tätigkeiten eines Renntierhierten wie folgt: U mań pàdēñe tề'np msèmmańt's' Ich war bei den davonfliehenden Renntieren' (Mat 359). ${ }^{1}$ — In einer

1 In Castréns Konjugationsparadigmen wird das Gerundium auf manś, manź, mand als Form der 1. Sg. der II. Zeit des Konjunktivs angeführt (Gr. S. 393, vgl. S. 225). Bei Sprogis sehen wir in den Paradigmen mehrerer Kaniner Zeitwörter das Gerundium auf monź als entsprechende Form des Indikativs (ALH II 159, 160, 162, 165, 168, 171, 177). Die entsprechende Form der wie Verba konjugierten Nomina hat auch die Endung monź: ('Wirt') jerovomonź, ('jung, Kind') nač(e)kemonź (ebd. 158-159). 
Angabe aus der MS-Mundart tritt die Endung des Gerundiums an einen Stamm mit dem Suffix *je (i) bzw. ní "tamnp

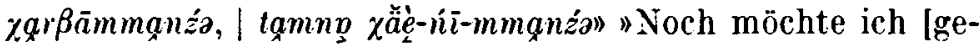
hen,] noch würde ich gehen" (275). Auch der mit dem Suffix * na gebildete Verbalstamm hat eine Gerundiumform in prä-

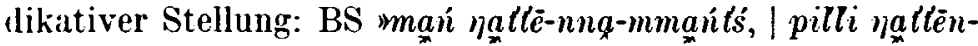
nqummanit's" \& Ich habe gewartet, | gänzlich habe ich gewartet" $(291)$.

Auch das Gerundium auf mans' kommt ziemlich oft in adverbialer Funktion vor: (C) jili-b-mansj ( jili-ptsi-mansi) hajeh '[sie] gingen auf Renntierjagd' (124); »njere, kāntam :ah | varaukumansi" "Mutter ich gehe | Birkenspanbäume suchen"

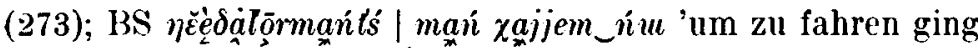

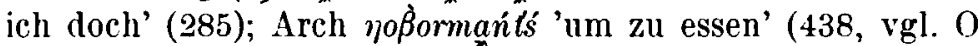
105); U jōrmańt'se $\chi \bar{a} n t t a \bar{\beta} \beta \beta_{3}$ ' 'wir begeben uns das Zugnetz zu

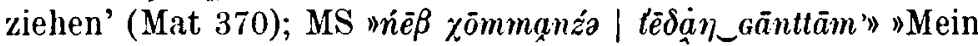
Weib zu holen | begebe ich mich jetzt» (292; vgl. O 1, 44, 49);

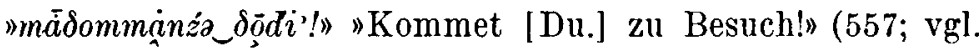
558). Vgl. noch Kan mūsśselmanź msèj ' 'er begab sich auf die Umzugsfahrt' (218); U mànssārmāñ t's' 'besuchen' (Mat 352; vgl. BS 574, 575, 'T Mat 417); MB (BS) ṕpurmant's $\chi \bar{a} n t t a \overline{~ ' e r ' ~}$ greht ... jagen' [eig. suchen] (Mat 372); O sainno-rmmañ t's 'in den Kampf' (17; vgl. BS 288); OD śūurmeñt'se 'um zu begraben' (188) usw. ${ }^{1}$

1 Erkki Itkonen befasst sich in seiner Studie .Das Perfekt des Partizips im Lappischen* (MSFOu XCIII, 241-304) eingehend mit de. Herkunft des lappischen verbalnomenbildenden Suffixes maŕdžs, ferner mit dem Verhāltnis der Verbalnomina auf marid ̌́e und der Verbalnomina auf ${ }^{*} m e$ zueinander, wie auch mit der syntaktischen Funktion der beiden Verbalnomina: beide können als Prădikate in verneinenden Sătzen bzw. in solchen mit hypothetischem Inhalt erscheinen. Obwohl E. Itkonens Konzeption, auf deren Grundlage er das lappische Gerundium erklärt, wesentlich von meiner Auffassung abweicht, kann die auffallende Ähnlichkeit, die sich in den formalen Eigenheiten und den syntaktischen Funktionen des lappischen Verbalnomens und des jurakischen Gerundiums beobachten lāsst, unserer Aufmerksamkeit nicht entgehen. $\mathrm{Ob}$ ein genetischer Zusammenhang zwischen den Endungen der lappischen und der jurakischen Formen besteht, können nur die Lappologen entscheiden. 
Desiderative Verbalnomina mit dem Suffix ${ }^{*} k$.

Verbalnomen a uf *ka, *ke

Zum Ausdruck des Imperativs, der strengen Aufforderung, gebraucht die juraksamojedische Sprache die Aoristformen für die 2. Person im Singular, Dual und Plural des Indikativs, das Zeichen des Befehls ist aber der auffordernde Ton: $O$

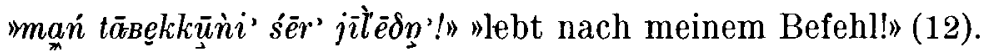
Auch die entsprechenden Formen der nominal gearteten Verbalnomina können eine energische Aufforderung ausdrücken:

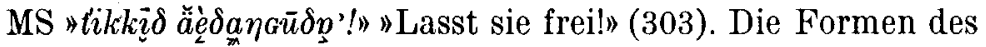
Konjunktivs, des Konditionalis und des Optativs werden oft zum Ausdruck einer Bitte, eines Wunsches gebraucht. Auch die mit dem Suffix $* k$. gebildeten Verbalnomina drücken ein Desiderativum aus. Die Formen des Desiderativs kommen nur in der Rede von Personen vor. Da die eventuelle Erfüllung der Bitte, des Wunsches, des Verlangens von dem Gesichtspunkt des Sprechers aus nur in der Zukunft erfolgen kann, können die das Desiderativum ausdrückenden Formen weder Vergangenheit noch Gegenwart bezeichnen.

In einem Teil der Angaben erscheint der Kehlkopfverschluss am Ende der Form der 2. Sg.: O $" t \bar{u}$ '! " Komme ...

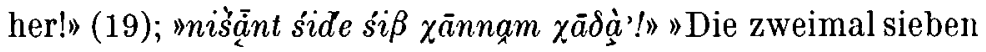
Blutopfer deines Vaters [eig. deinem Vater] töte!» (165). Wenn wir die Imperative vom selkupischen Typ kuk! [stirb!] (Mat 38 , ferner das Zeichen $k$. des kamassischen Imperativs ins Auge fassen, können wir für wahrscheinlich halten, dass in den Formen dieses Typs, - zumindest in einem Teil der Angaben - der Kehlkopfverschluss ein Suffix * $k$. ersetzt. In den Kaniner Aufzeichnungen von Budenz erscheint das Element $k$. am Ende des Hauptverbs einer verbietenden Konstruktion "nūn udn tānak!» ['mach dich nicht auf den Weg'] (NyK XXII, 85). Auch in Castréns Texten finden wir einen Satz, in dessen Prädikat vor dem Px. der 1. Sg. ein Element *k erscheint: "tjuki $j \bar{a} d \mid \bar{a} n i n$ gäe-h-ua | tūmari jān!» "Von dieser Stelle | lasst uns wieder gehen | an euren Ausgangsort!» (255). Beachten wir sämtliche Angaben, so scheint es wahr- 
scheinlich, dass das Suffix auch ein vokalisches Element hatte, nach dessen Schwund auch das Element ${ }^{*} k$ eingebüsst wurde.

Das Suffix *ka, das mit Suffixen, die Irrealität und den Zustand ausdrücken, verbunden werden kann, bildet aus intransitiven, transitiven und passiven Verben Verbalnomina zum Ausdruck der Möglichkeit, des Wunsches und der Absicht. Das Possessivsuffix deutet das Subjekt des mit dem

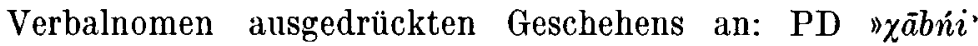

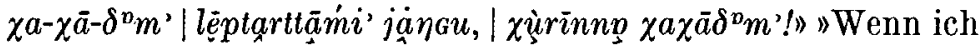
sterbe, so lass sterben, | von mir abhängig ist es nicht, | ir-

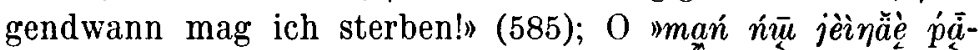

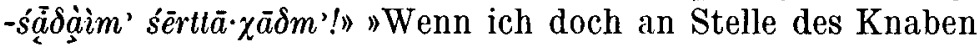

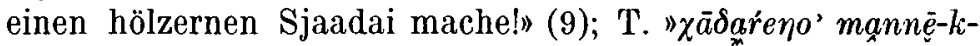
$k a-m \bar{\imath}-m m p ! " ~ " L a s s$ uns [Du.] wirklich versuchen!» (93); O

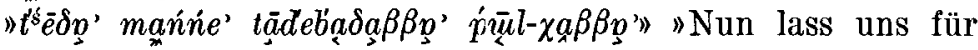

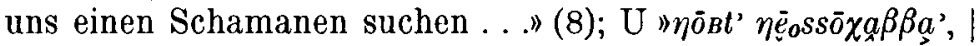
$\eta \bar{o}$ kann sammen!» (Mat 388). In einer Angabe aus dem OD-Dialekt ist $m m p$ nach dem Suffix *ka entweder ein zustandsbezeichnendes Suffix oder ein verstärkendes Element. Eine Angabe OD $p \approx \grave{e} \dddot{j} j \bar{e} \delta p j \bar{a} \dot{a} k k_{\wedge}^{a} \chi a \lambda_{m m p}$ 'Seine Stirn begann zu jucken' (74) deutet darauf hin, dass das Element ${ }^{*} k a$ nach seiner Grundfunktion ein Inchoativsuffix ist. (Auf dieselbe Grundfunktion deutet auch das Suffix ${ }^{*} k a,{ }^{*} k a l$ in sekundären Verbalstämmen.)

Sehr aufschlussreich sind die vom Stamm min- 'gehen' stammenden, mit dem Suffix ${ }^{*} k a$ gebildeten Verbalnomina, die nur in der Übersetzung der Texte Präsens-Bedeutung haben. Die aus den tundra- und waldjurakischen Dialekten bekannten Stämme min-, mina- sind aus der Verbindung ${ }^{*}$ min $+k a$ entstanden (vgl. Beitr. 33-34). In Castréns Texten haben sowohl der Stamm als auch das Suffix noch ihre Selbständigkeit bewahrt: "āni jān min-ga-m» "an einen anderen Platz gehe ich" (93); "amgaes mingan?" "Warum gehst du?"

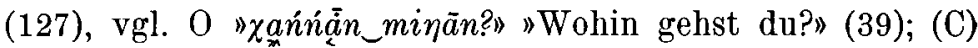
siu jāle mingah 'sieben Tage geht sie' (36; vgl. 201, 208); "nieleuansi minga-ua» "Jetzt gehen wir . . . [ihn] verheiraten" (64), 


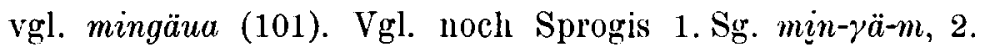

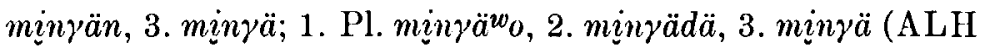
II, 175). In Lehtisalos Angaben begegnet der Stamm mina-, im $\mathrm{Wb}$ (276) ist aber eine Angabe aus der W Nj-Mundart

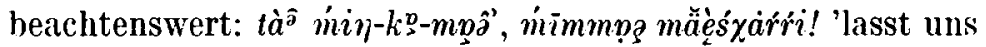
gehen, man muss gehen!'

Für Verbalnomina mit dem Suffix $* k a+j \varepsilon$ (in denen auch ein zweites Suffix $* j \varepsilon$ erscheinen kann) haben wir Angaben besonders aus dem PD-Dialekt. Das Verbalnomen mit nominalem Charakter dient zum nachdrücklichen Ausdruck des

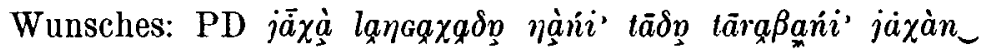

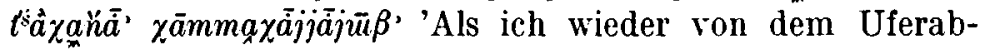
sturz mit dem Fuss stiess, stieg ich gern zur anderen Seite des Flusses hinab' (Mat 413; vgl. 194, Arch 179, usw.); OD

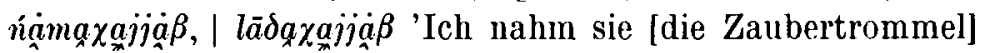
gern in meine Hand, gern trommelte ich' (193); Arch jēśse

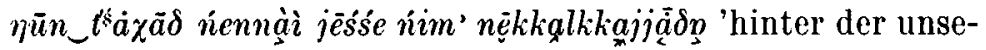
ren Zeltstange den silbernen Gürtel gern ergriff er' (173) usw. ${ }^{1}$

Mit dem zusammengesetzten Suffix $-\chi a \beta \beta a-\left(<{ }^{*} k a+p a\right)$ entstehen auch Verbalnomina mit nominalem Charakter; diese haben sehr selten prädikative Funktion: BS ssidem

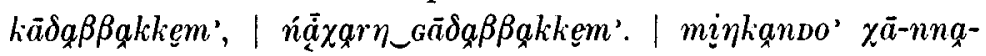
$\chi a \beta \beta x^{\prime}$ " "Zwei habe ich wohl getötet, | drei dürfte ich getötet haben | am Essen sind sie gestorben" (324-325). Der Kehlkopfverschluss am Wortende ersetzt das abgeschliffene Pos-

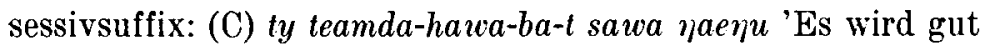
sein, wenn du das Renntier kaufst' (WbAnh. 373); 0 'märp’'

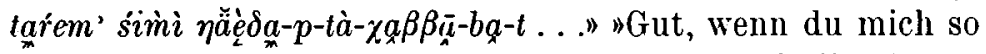
schickst ..." (240). Die Dehnung des Vokals im Suffix deutet die Verschmelzung des Suffixes ${ }^{*} j \varepsilon(i)$ an. - Die mit Kasussuffixen versehenen Formen des Verbalnomens kommen nur

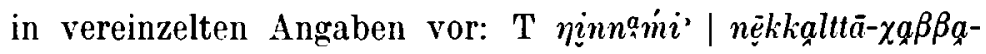

${ }^{1}$ Die Deutung des Verbalnomens ist irrig in der folgenden Angabe: BS e r s c h l a ge nl" (357) Es handelt sich um das Verbalnomen des Verbs $\chi \bar{a}$ - 'sterben', das die Suffixe $p+t a+\chi a j j e$ enthält; die Bedeutung des Satzes ist also 'das Alter hat er, es wäre gut, wenn er stürbe' oder 'er könnte schon sterben'. 
zañi' .. . 'Meinen Bogen, | während ich ihn doch spannte ...'

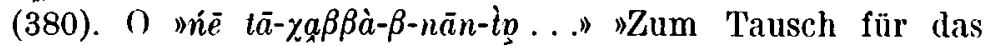
Weib ..." (455). - Das Verbalnomen kann auch als Gerundium im Satz erscheinen. Seine Bedeutung entspricht in dieser Eigenschaft ungefähr der Bedentung des ungarischen Verbaladverbs mit den Endungen ra, ve, tán, vén: 0 t'ikliz jēśśs

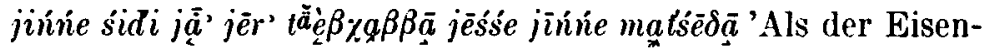
draht zwischen zwei Ländern angekommen, hielt er plötzlich

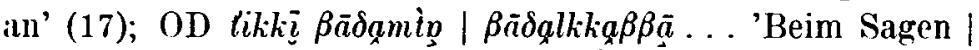

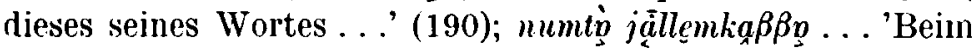
Tagesanbruch ...' (93; vgl. 94, 101). - Das Verbalnomen kommt auch in figura etymologica- Konstruktionen vor: 0

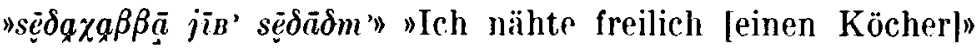
(149). ${ }^{1}$

Der Gebrauch der mit dem Suffix *ke ge bild et e $n$ Verbalnomina ist sehr beschränkt. Für seine prädikative Funktion kann ich nur die Verbalnomina anführen, die aus dem Verb mit der Bedeutung 'fortgehen' stammen; in diesen ist das Subjekt des Geschehens immer die

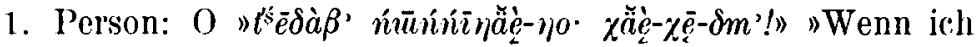
aber jetzt als Polartaucher fortgehe!» (36); (C) "häheni ( häeni $\sim$ häehanj)! | atseki wuenikom | team-da-ha-ni $(\sim$ teamdanjih)!" "Lass uns [Du.] fortgehen! | Den Hund des Knaben

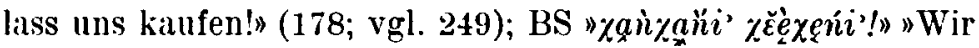
wollen zu meinem Schlitten gehen!" (333; vgl. Arch 426); Arch

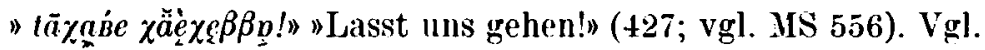
noch WLj kădde zemmpâ'! 'lassen wir ihn zurück!' (Wb 164).

- Das Suffix * $k \varepsilon$ wird an den Konjunktivstamm angehängt im folgenden Satz: (C) male heä-ji-ke-nje'eh 'Schon sind wir' im Wegfahren begriffen' (279). Aus dem WNi-Dialekt stammt

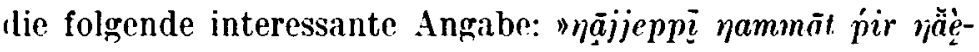

1 Zum nachdrücklichen Ausdruck der Irrealität des Geschehens kann auch das Suffix - $\chi a \beta \beta a$ - an das Nomen, das Pronomen, das Adverb usw.

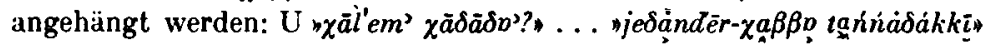
"Habt ihr Fisch bekommen?.... *Ein Topf voll mag es sein* (Mat 372,

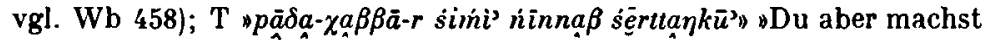

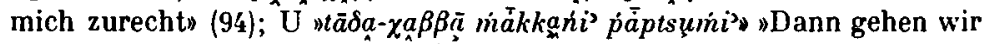

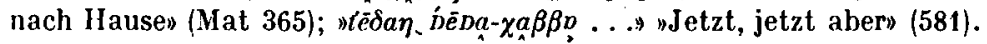


$\chi \varepsilon^{-m m a \bar{a}-p-t p} \eta a m t m p p^{\prime} ! " ~ " W e n n$ du imstande bist roh zu essen,

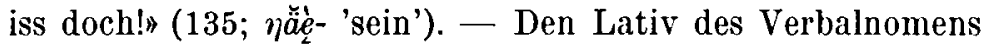
auf $-\chi \rho \beta \beta a-\left(\mathrm{vgl}-\chi_{\alpha} \beta \beta a-\right)$ sehen wir in der folgenden Angabe:

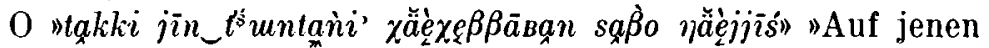
Sandrücken wäre es für mich gut zu gehen" (153).

Für das Verbalnomen a uf $\eta k a$ gibt es nur vereinzelte Angaben. Aus der Verbindung des Verbalstammes min- 'gehen' und des Suffixes *ka ist, wie bereits weiter oben zu sehen, der sekundäre Stamm mina- entstanden. Unter Berücksichtigung dessen können wir das in den Texten vorkommende Suffix $\eta k a$ so auffassen, dass das Element $\eta$ ein sekundärer Laut ist und das zusammengesetzte Suffix auf die Verbindung $* n$. $+k$. zurückgeführt werden kann. In Castréns Texten und waldjurakischen Angaben ist die Verbindung $n+k a(g a)$ keine orthographische Eigentümlichkeit und kann nicht mechanisch durch das Suffix na ersetzt werden (vgl. Mat 313-316). Vereinzelte Angaben lassen sich

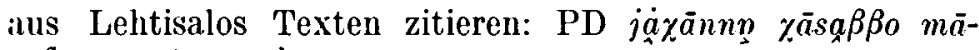

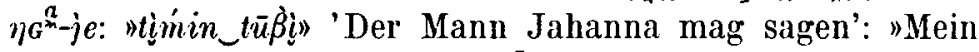

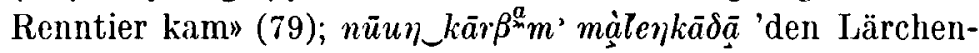
baum des Himmels | zerschlug er' (Mat 455; Zaubergesang). Man findet einige Angaben auch in Gesängen aus der BS- und

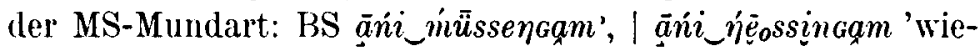
der begab ich mich auf die Umzugsfahrt, | abermals lagerte ich'

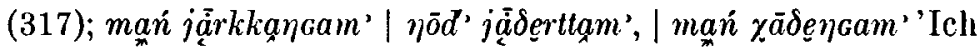
fing lein mit Müh und Not Gehendes | ich tötete' (ebd.); "pēt-

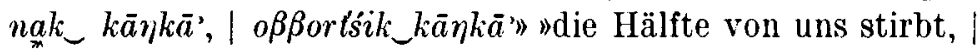
stirbt Hungers» (313); vgl. MS tē sie [Du.] noch' (355).

Verbalnomen a uf ${ }^{*} k a r,{ }^{*} k \varepsilon r$ (del Precativ)

Nach Castrén ist das Suffix des Precativs ein Moduszeichen (Gr. § 471). Eigentlich ist der Precativ auch ein Verbalnomen, dessen Suffix dadurch entstanden ist, dass das Suffix *ka, ${ }^{*} k \varepsilon$ mit dem intensiv-effektiven Suffix $r$. verbunden wurde. Eben deshalb dient der Precativ zum emphatischen Aus- 
druck des Wunsches, des Verlangens. Sein Suffix verbindet sich oft mit dem Suffix des Konditionalis und des Desiderativs. Der Precativ hat keine Formen für die dritte Person. Die Bedeutungsabschattungen werden veranschaulicht durch

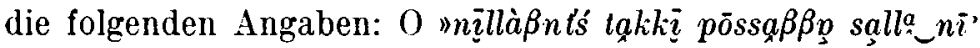

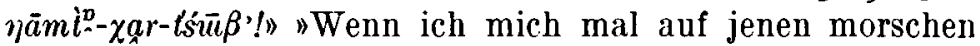
Baumstumpf zum Ausruhen setzte!" (153; vgl. 40); "takki .

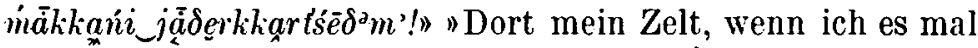

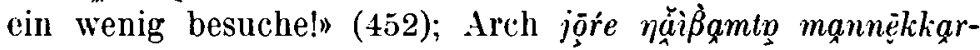
t'sés $\delta m^{\prime}$ 'ich versuche mal, ob es seicht ist' (434); WNi "tupkp

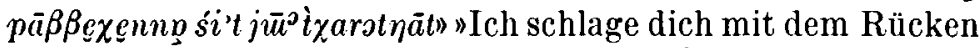

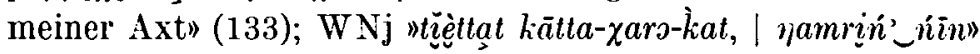
"Wenn ich dir ein Renntier tötete, | da willst ja essen» (415). Am Ende der Formen der 2. Sg. deutet der Kehlkopfverschluss das abgeschliffene Possessivsuffix an, das in einigen Angaben doch noch vorhanden ist: O "tà $\chi$ àrt!" "Gib doch!"

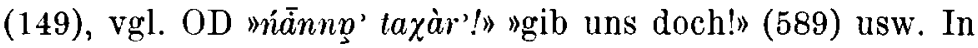
verneinenden Fügungen erscheint das Suffix *kar und der Kehlkopfverschluss am Ende des Hauptverbs: WNi »śe'ej nin-

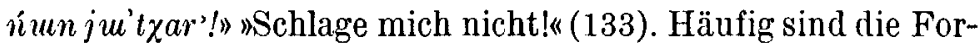

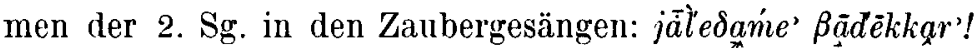
'Sage mir doch Licht!' (Mat 458). In einer Angabe stammt die Precativform aus dem Passivstamm: Pứŕe-Fl. "tedpmpọ̀ '।

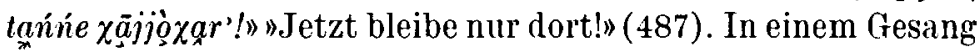
von cinem Zauberer ist das Suffix des Precativs zweimal an

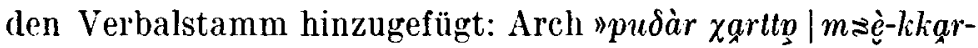
kkar-t!»'Du selbst | nimm doch!' (182). Auch für die 2. Pers. Plur. haben wir eine Angabe aus einem Zaubergesang: 0

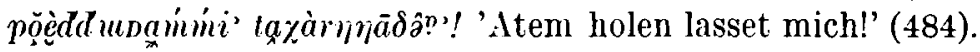
Hit dem Suffix *ta verbunden erscheint das Suffix *kar auch im Akkusativ eines Nomens verbalnominaler Herkunft:

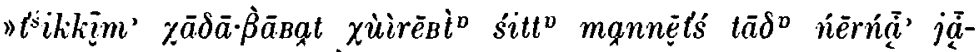

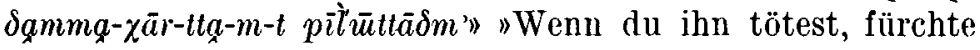
ich mich nicht nur, wenn ich dich sehe, sondern von da an werde ich auch deine Spuren fürchten» (50).

Für d a s $\mathrm{S}$ u f f i $*_{k \varepsilon r}$ gibt es weniger Angaben. Ebenso wie im Falle des Suffixes $* k \varepsilon$ kann ich die hierhergehörigen Formen des Verbs mit der Bedeutung 'fortgehen' anführen. 
In diesen Formen ist das Suffix *ker mit dem Suffix des Konditionalis verbunden: (C) "niernjangi müuda $(\sim$ müudaha)| manj häertsem ( hä-her-tse-d-m)" "Zu den vorderen Karawa-

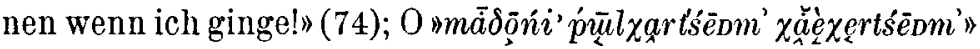
"Wenn ich doch gehe meine Zelte zu suchen" (449; vgl. O 1,

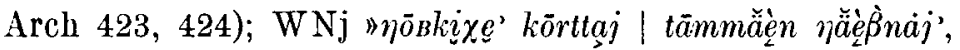

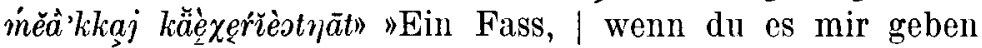
würdest, | begebe ich mich nach Hause" (409-410); T "pin-

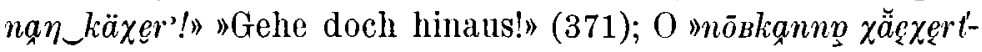
śen $i$ !" "Lass uns zusammen gehen!" (462; vgl. Puŕré-Fl. 493);

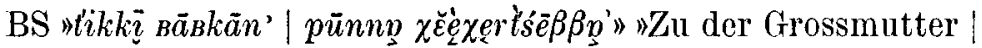
begeben wir uns später" (578). Die vereinzelten Angaben, in denen das Suffix in der Form - $\chi \bar{o} r$ - erscheint, sind nicht ein-

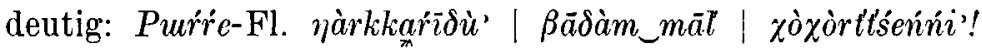
'Nur Grosse von ihnen | am Ende des Wortes | lasst uns fin-

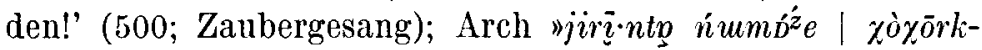
kart!" "finde doch | den Namen deines Grossvaters" (184). In diesen Angaben kann das Suffix - $\chi \bar{o} r$ - auf eine ursprüngliche Form $\chi$ ar zurückgeführt werden, mit Rücksicht auf den $\bar{o}-$ Vokal des Stammes $\chi \bar{o}$ und auf die velarisierende und längende Wirkung des Suffixes $r$. Doch könnte man das Suffix eventuell aus einer Verbindung ${ }^{*} k \bar{o}+r$. erklären. - In einer Form der 3.Sg. hat das Suffix *kar vielleicht inchoative Bedeutung:

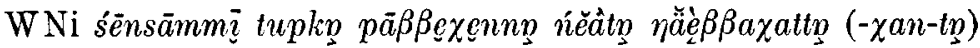

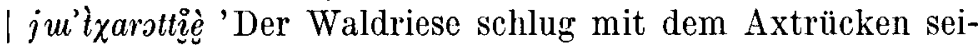
nen Kameraden auf den Kopf' (133); es lässt sich aber das Prädikat auch in dieser Weise deuten: 'er mag [auf den Kopf] schlagen'.

$$
\text { Das Verbalnomen a uf *kiu }(* k \bar{o})^{1}
$$

In den Konjugationsparadigmen von Sprogis sind die Futur- ("dritte Zeit») Formen des Verbs mit der Bedeutung 'durchschneiden' folgende: 1. Sg. made-gū (-gum), 2. madegur (-gun), madegudä (-gu) nsw. Die "negative Form" lautet aber

1 Die Ableitungssuffixe ${ }^{*} k a,{ }^{*} k \varepsilon,{ }^{*} k \bar{o},\left({ }^{*} k \bar{u}\right)$ haben Entsprechungen im Finnischen und im Lappischen, vgl. L. Hakulinen SKRK ${ }^{3}$ S. 201 -202 ; M. Korhonen: MSFOu 143: 155-156, 163-164, 169-171. 
niu madegu usw. (ALH II, 171). Im Wb (438) findet sich die folgende Angabe: W Nj šì̀rottp $p^{2}-k k \bar{u}-m p s s^{\prime}$ 'ich wollte machen, hätte gemacht'. In Lehtisalos Texten kommt das Verbalno-

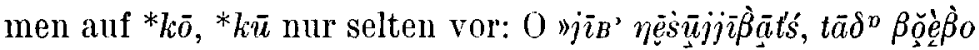

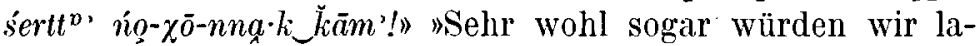
gern, wenn wir nur nicht dann in eine schlimme Angelegen-

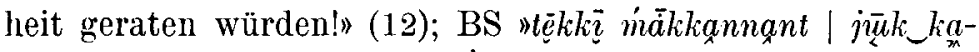

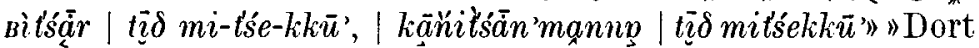
in deinem Zelte / deine zehn Knechte / verkaufen deine Renntiere, | an die Leute von Kanin | verkaufen sie deine Renntiere" (567). Das Verbalnomen hat nominalen Charakter in

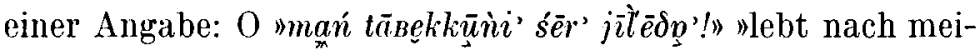
nem Befehl!» (12). Aus dem Waldjurakischen gibt es mehrere

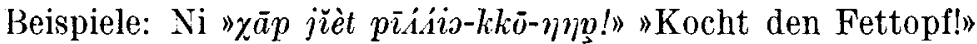
(83; vgl. 133, 145, Nj 411). Aus Castréns Texten haben wir mehr Angaben. In einem Gesang begegnet uns das Verbalnomen auf *ko als Gerundium: tarsem namda-ho-si | pin sanojiu'ah 'Beim Hören von derartigem | sprang ich hinaus' (301302). Auch in prädikativer Funktion kommt das Verbalnomen vor: "amgahädando | hōnar-kū" "Über etwas schickt sie Botschaft" (87), "tōja maran | tasīn gām-gu-di!" "auf den (Strand-) Sand des grossen Sees | geht [Du.] hinab!» (137). Es gibt Angaben auch für das Verbalnomen auf $* k \bar{u}$, bzw. $* k \bar{u}+$ ta, das aus dem Verb mit der Bedeutung 'verfolgen, nachjagen' gebildet ist: atseki njüdüjum | nießemda njuo-ro-ku-a-da 'der jüngere Knabe | jagt seiner Mutter nach' (14; vgl. 45, 214); tijki jäd | hōndie darjau | njuoroku'udi' | tarem njuoro-kutadi'eh. . . 'von dieser Stelle | wie das Schneehuhn | jagen sie (zu zweien) ihnen nach. | Während sie so nachjagen ...' (202; vgl. 205). Bei Lehtisalo findet sich der Lativ des Verbalnomens auf

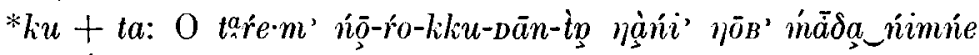
nadime 'während er so verfolgte, kam er wieder zu einem Zelt' (172). Wenn wir die Verbalnomina auf $\bar{o}, \bar{u}$ sowie $p t s \bar{u}$ betrachten, können wir feststellen, dass das Suffix ${ }^{*} k \bar{u},{ }^{*} k \bar{o}$ auf eine Verbindung ${ }^{*} k a \sim k \varepsilon+\beta$ zurückgeführt werden kamn und dass es Passivität bezeichnet. ${ }^{1}$

1 In Castréns selkupischem Material kommt die Verbform mit dem Suffix $k u$ vor: N konda-ku-ak (Mat 37, vgl. 199-200). 
In vereinzelten Angaben erscheint das Verbalnomen auf

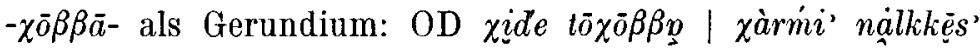
ni $\beta \beta \bar{o} \beta$ ' 'Während die Schüssel doch kam, | zog ich mein Mes-

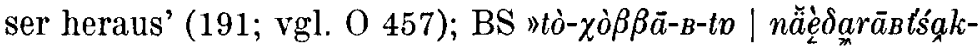
kem'" "Wenn er kommt, | so schicke ich ihn vielleicht" (202). ${ }^{1}$

\section{Das Verbalnomen a u $\eta k \bar{u}$}

Der Gebrauch des mit dem Suffix $* k \bar{u}(k \bar{o})$ gebildeten Verbalnomens ist, wie weiter oben ausgeführt, ziemlich beschränkt. Die Zahl der Angaben vervielfacht sich aber, wenn wir die Verbalnomina auf $\eta k \bar{u}$ in Betracht ziehen: das Suffix $\eta k \bar{u}$ ist auf eine Verbindung $*_{n}+k \bar{u}$ zurückzuführen. (Das Element $\eta$ ist also ein sekundärer Laut aus ${ }^{*} n$. vor $k$.) Das Subjekt des mit dem passivischen Verbalnomen ausgedrückten Geschehens wird durch das Possessivsuffix bezeichnet. Im Band "Materialien" teilte Lehtisalo sechzehn jurakische Texte mit, aus denen wir die Lebensweise der Juraksamojeden kennenlernen können. Diese Beschreibungen sind nicht nur wegen ihres Inhalts interessant, sie sind auch sprachlich sehr aufschlussreich: der Gewährsmann gebraucht in seinen Mitteilungen - obwohl er Geschehnisse erzählt, an denen er selbst aktiv beteiligt zu sein pflegt - Formen, die Passivität und Irrealität bezeichnen, in erster Linie solche mit der Endung $\eta k \bar{u}$. In der kurzen Erzählung des Renntierhierten (Mat 355-356) finden sich fünfunddreissig Verbalnomina auf $\eta k \bar{u}$. Die Erzählung beginnt: U ńųurt'té jōrkulla

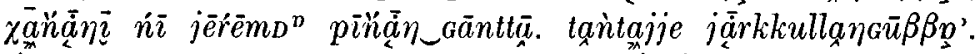

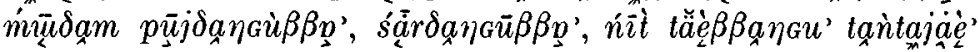

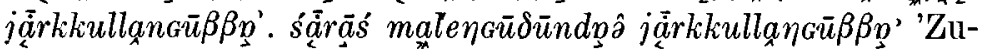
erst treiben wir die Renntiere in einen von den Renntierschlitten gebildeten Halbkreis. Wer nicht hineingerät, begibt sich fort. Gleich fangen wir [es] mit dem Lasso. Wir bilden

${ }^{1}$ Das Suffix $-\chi \bar{o} \beta \beta a-$ kann auch zu einem Nomen hinzugefügt wer-

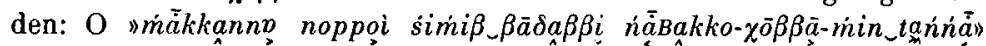
"Im Zelte befindet sich nur meine ältere Schwester, die mich aufgezógen hat" (463). 
einen Renntierzug, spannen die Renntiere dafür an. Wenn es nicht genug gibt, fangen wir gleich mit dem Lasso. Wenn sie das Anspannen beendet haben, fangen sie mit dem Lasso' (Mat 355).

Die Verbalnomina auf $\eta k \bar{u}$ - ob sie nun aus intransitiven oder transitiven Zeitwörtern stammen - kommen sehr oft in den Reden von Personen vor. Die Beispiele veranschauli-

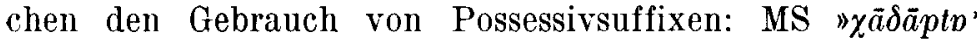

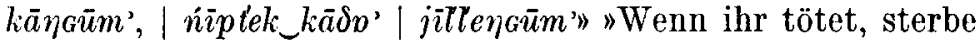
ich, | tötet ihr nicht, | lebe ich" (304); O "mań täę $\beta \eta \bar{u}_{\bar{u}} \beta$ " " )ich komme zurück" (211); (C) "hāptm dāngum $(\sim$ dātam $)$ » "einen

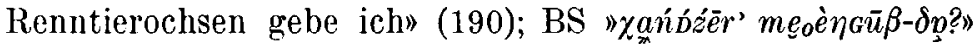
"Was tue ich [d.h. was kann ich dafür]" (325), - $\mathrm{N} \beta \approx \grave{s} s \bar{s} k k u$,

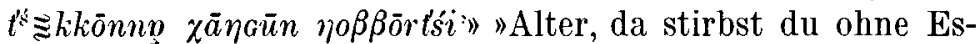

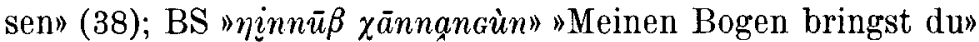

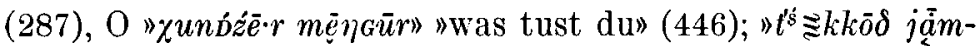

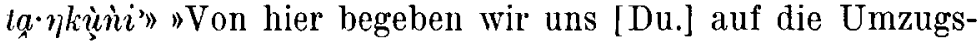
fahrt" (164); OD "jìri tuBkam' tällequami'" "Wir stehlen die

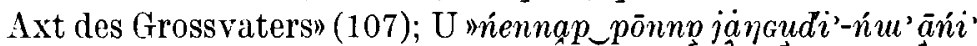

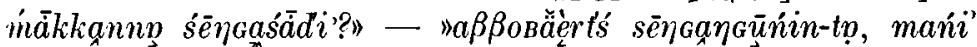
juховӧrnañi" "Sehr lange wart ihr fort. Habt ihr in einem anderen Zelt übernachtet?" - "Warum würden wir [Du.] übernach-

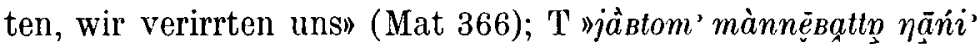

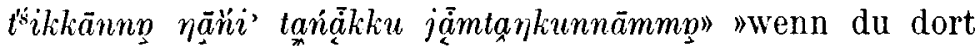
wieder Gänse siehst, werden wir dorthin übersiedeln" (Mat 417); (C) "hattarie hāpt | ānin gōnguda' | sidda taeurāngū" "den grauen Renntierochsen [Männername] ihr wieder findet

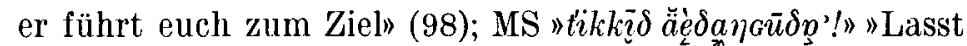

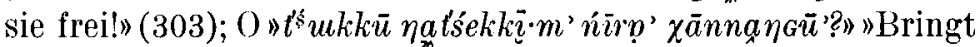
ihr nicht dieses Kind fort?» (446). - In den Formen der 3. Sg. erscheint - bei intransitiven und transitiven Verben gleichermassen - z.T. der Verbalnomenstamm, z.T. das Verbalnomen + Px. der 3. Sg.: U jảa $\delta a \eta \eta_{G} \bar{u}, j a \dot{a} \delta a \eta \eta_{a} \bar{u}$ 'Er läuft Ski, läuft Ski' (Mat 364), vgl. jąं $\delta a \eta a \bar{u} \delta p ~ j a \bar{a} \delta a \eta a \bar{u} \delta p$ id. (ebd. 365);

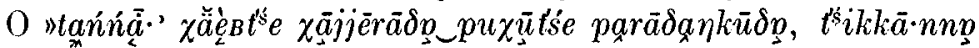
$\chi \bar{a} \eta k \bar{u}$ " "Wenn er sich dorthin begibt, verbrennt ihn die Sonnen-

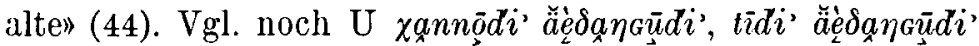
'Sic [Du.] spannen den Schlitten aus, lassen die Renntiere los' 


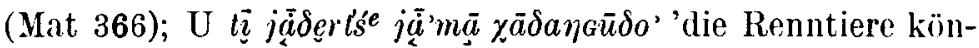
nen nicht gehen, sie töteten sie' (Mat 351).

In vielen Fällen verbindet sich das Suffix $\eta k \bar{u}$ mit den Suffixen *ma, *mpa, *pa, *'a, *ta, ferner mit dem Element. $k+k \dot{e}, k+\bar{i}$.

Das desiderative Verbalnomen auf *ta und der desiderative Nominativ, Akliusativ und Lativ der Nomina

In einem Gesang aus dem MS-Dialekt gibt der Vater seinem Sohn Ratschläge. Nach den als Prädikat gebrauchten Verbalnomina des Konditionalis, des Potentialis, des Optativs, sowie nach den passivischen Formen im ersten Teil des Zitates, folgen Prädikative mit dem Suffix * $t a: " t e \bar{\delta} \delta v^{\prime} t u k k \bar{o} \delta$

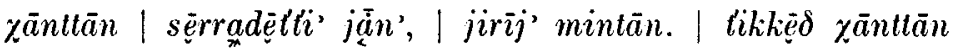

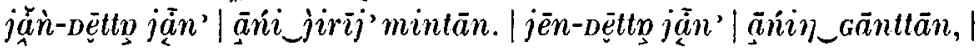
ăni_jirij mintān." "Von hier begibst du dich jetzt $\mid$ in das I and der Witwer-Wirte, | einen Monat gehst du. | Von dort begibst du dich | in das Land des Iand-Wirtes, | wieder cinen Monat gehst du. | In das Liand des Sehnen-Wirtes | wieder gehst du, | abermals gehst du einen Monat» (298-299). Die Bedentung der mit dem Suffix $\boldsymbol{\imath} \vec{a}$ gebildeten Formen ist etwa 'du könntest weggehen [von dort]' oder 'es wäre gut, wenn du weggingest' usw. Das Suffix tā lässt sich auf die Verbindung $* t a+j \varepsilon(i)$ zurückführen. In den Beschreibungen der Lebensweise der Juraksamojeden kommen oft Prädikate mit dem Suffix * $k_{k} \bar{u}+t a$ bzw. *ta vor. Die Zeitwörter 'kommen', 'grehen', 'fortgehen', 'sprechen' begegnen regelmässig in der 3. Sg. als tuttā, mintā, yãnttā, mantã. Aus denselben Texten haben wir auch eine Angabe für die sonst selten vorkommende

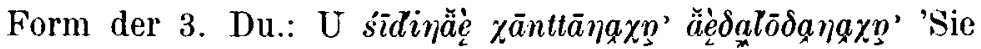
fahren zu zweit fort, fahren' (Mat 364). Auch für den prädikativen Gebrauch der aus transitiven Zeitwörtern stammenden Verbalnomina auf $t \bar{a}$ gibt es Belege: 0 " $\chi \dot{i} \dot{b} \dot{a} \delta$ amaêm tat$t \bar{a} \delta m$ '?" "Von wem soll ich etwas bringen?" (155); T "xànnámin

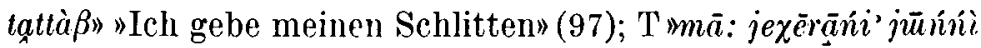
tattäni $i$ " "Er sagte: wir [Du.] bringen fremdartige Pferde» 


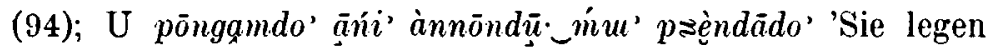
ihr Zugnetz wieder in ihr Boot' (Mat 371). Häufung von Ableitungssuffixen sehen wir in einem Zitat: $O " \beta \approx e ̀ s s c k k \bar{o} \delta v$ $\eta a \tilde{a} e_{c} \delta a-p-t \bar{a}-n n a-k k \bar{e}-\delta p$ " "Ihr Alter hat sie wohl geschickt" (28).

Eine beachtenswerte Angabe liegt in einem Gesang vor:

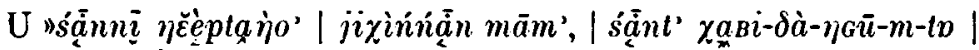

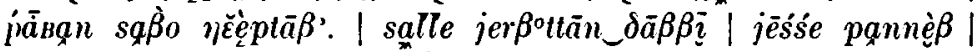

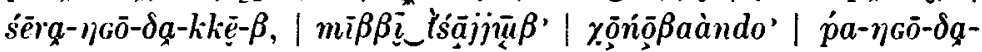

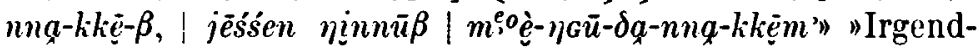
wann | dachte ich, | wie lange bin ich Knecht, | mag es gut sein anzufangen? | Den rom Fürsten von Obdorsk gegebenen | Panzer | werde ich wohl anziehen, | an die versammelten Krieger | mache ich mich wohl heran, | wenn sie schlafen, | ich nehme wohl / meine Flinte" (Mat 389). In diesem Zitat. finden wir vier Formen, in denen die Suffixverbindung $t \bar{a}+$ $\eta k \bar{u}(+n n a+k k \bar{e})$ in Formen der 1. Sg. mit prädikativer Funktion erscheint; darunter das aus dem Wort $\chi \alpha_{m}{ }_{i}$ ' Knecht' stammende Verbalnomen auf $t \bar{a}+\eta a \bar{u}$. In einem Zitat aus einer Sage begegnen uns drei Nomina, die wie Zeitwörter konjugiert sind, zwei von diesen nelımen das Suffix *ta an

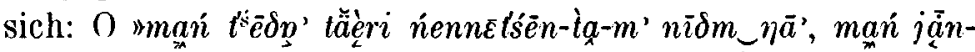

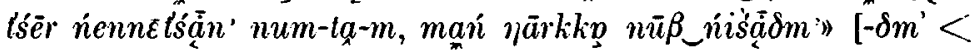
$\left.* t \dot{a}+m^{\prime}\right]$ "Nun bin ich kein belangloser Mensch, ich bin der Himmelsgott für den Menschen der Erde, ich bin der grosse Vater des Himmels" (13).

Diese Angaben haben meine Aufmerksamkeit auf die nominalen Formen gelenkt, die ein Element *ta und Possessirsuffixe enthalten und die als Subjekte, unbezeichnete und bezeichnete Objekte, sowie als Lative erscheinen. Diese Formen hat Castrón unter der Bezeichnung "Objectaffixe" behandelt (Gr. $\S 445$, vgl. $\$ 377-378,388-389$ ) und festgestellt, dass das Grundelement der Objektaffixe "das Affix der dritten Person Singularis» ist. In Kenntnis der erheblichen determinierenden Rolle des Px. der 3. Sg. sahen wir uns nicht veranlasst, die Richtigkeit dieser Feststellung anzuzweifeln. Ich selbst habe die Benennung "determinierende Deklination" der Nomina in einer Arbeit gebraucht und sagte aus, dass die betreffenden Formen ein Desiderativum 
bezeichnen (ALH X, 55-92). Nach meiner heutigen Auffassung drückt gerade das Suffix *ta das Desiderativum aus; die Possessivsuffixe sind identisch mit den Possessivsuffixen, die den Nomina und Verbalnomina angehängt werden. Suffixe, die Irrealität bezeichnen, können - wie erwähnt (S. 189 ff.) - als "determinierende» Elemente mit Nomina verbunden werden; so überrascht es nicht, dass auch das desiderative Suffix ${ }^{*} t a$ bei Nomina erscheint. Solche Nomina kommen in Zitaten, in der Sprache der Gesänge und der Sagen, in der Erzählung von hypothetischen Geschehnissen, von Bräuchen vor. Die jurakische Sprache drückt das konkrete Objekt nicht mit Desiderativformen aus: $O$ " $\chi a r^{\prime}-$

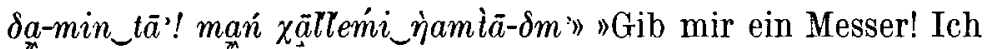
esse meinen Fisch» (172, vgl. 171), d.h. er will den Fisch [Akk.] essen, den er schon Besitz hat, und verlangt das Messer, das er bekommen möchte, das er nötig hätte: das letztere wird durch das desiderative Objekt ausgedrückt. ${ }^{1}$ (Anders P. Hajdú: NyK LXI, 238-246.)

Da in den erwähnten Arbeiten reichlich Materialien zur Verfügung stehen, will ich hier nur einige Beispielsätze anführen. Als Subjekt kommt das Desiderativnomen sehr selten

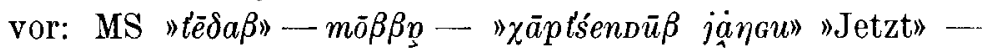
sagte er - wich werde nicht sterben" (281); die eigentliche Bedeutung des Satzes ist etwa: 'Tod für mich ist nicht vorhanden'. In den bezeichneten und unbezeichneten Akkusativen deutet das Possessivsuffix den künftigen Besitzer des

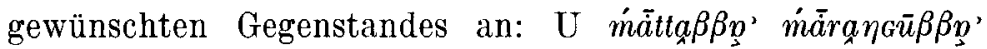

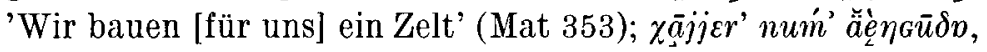

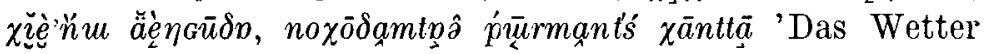
ist klar, es ist windstill, er geht einen Steinfuchs [für sich] jagen' (ebd. 372). Die Bedeutung des Desiderativ-Lativs

1 In der neueren linguistischen Literatur ist - meines Wissens - die von Prokofjev stammende Benennung "predestinative declension" (vgl. Bo Wickman FO S. 91) gebräuchlich im Zusammenhang mit den fraglichen Formen. Dieses Fachwort halte ich nicht für glücklich; es gibt im Bedeutungsinhalt des Verbs 'praedestino' keine solchen Elemente, die, wenn man auch den Kontext beachtet, die genaue Bedeutung der betreffenden Formen wiedergeben könnten. 
beleuchtet die folgende Angabe: MS "néénạit tẹdanop rååm', | jüjel tếgnop náăm!" " Fange ein Renntiere für deine Schwester, | fange ein Renntiere für deinen Schwiegersohn!» (280). Die Fügung 'als Renntiere deiner Schwester' drückt ein desideratives Besitzverhältnis aus.

Das Suffix *ta erscheint auch als ein Glied des zusammengesetzten Suffixes ${ }^{*} \beta+n$. $(+j \varepsilon)+t a$. Das ein solches Suffix enthaltende, nominal geartete, passivische Verbalnomen drückt die Notwendigkeit aus; seine Bedeutung steht der Bedeutung des ungarischen Verbalnomens auf andó, endó nahe. Das zusammengesetzte jurakische Suffix kann auch mit den Suffixen ${ }^{*} k a,{ }^{*} k a+p a,{ }^{*} n a$ verbunden werden und Possessivsuffixe an sich nehmen. Für die prädikative Funktion des Verbalnomens besitzen wir nur vereinzelte Angaben;

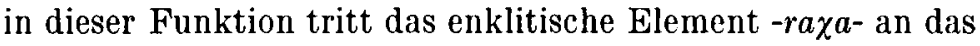
Verbalnomen: Arch $t_{\bar{e}} \delta \bar{a} \beta \quad \chi \bar{a}-\beta n t \bar{a}-r a \chi \chi \bar{a}-\delta m$ ' 'Jetzt sterbe ich offenbar' (433); vgl. (C) "ha-undā-rha-m" "Ganz als ob ich

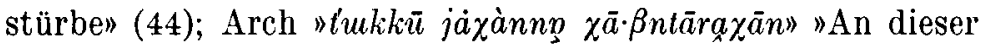

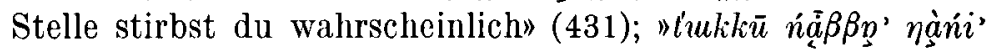
$\chi \bar{a} \beta$ ntäraxpg" "Dieser unser Kamerad stirbt wahrscheinlich" (436). Es sind mehr Angaben für den nominalen Gebrauch des Verbalnomens vorhanden: (C) häe-undia-do jānguh, I to-undã-do jānguh 'es gibt keinen, der geht, | es gibt keinen,

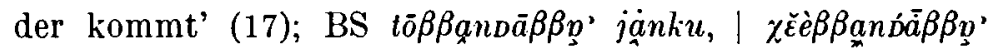
jäku 'Kommende haben wir nicht, | Gehende haben wir nicht' (328; vgl. Kan 215); (C) jinjan mam'ah: hā-vundā-na-u jāngo-u-n-gā-si-nju'uh 'Ich dachte: einen Totkranken | hatte

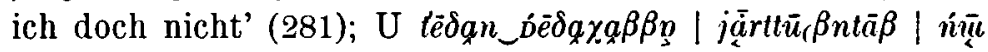
$\beta$ îne tanne' 'Jetzt, jetzt aber, mein zu beweinendes Kind gibt

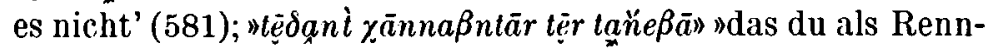
tier zu dir bringen sollst, ist da" (Mat 407); Arch " $\chi \bar{a} \beta n t a$. $^{*}$ nennętśácm jïltiptēm du gerettet” (434). Für den akkusativischen und lativischen Gebrauch des Verbalnomens haben wir Angaben aus dem

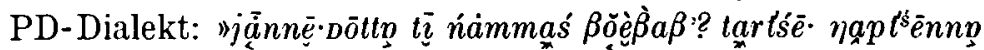

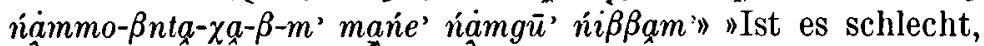
das Renntier des Verwandten zu fangen? Solch ein stinkendes Festzunehmendes nehmen wir ja fest» (Mat 408); PD " $\chi \bar{a} \beta$ - 


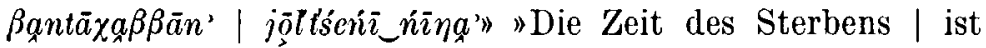
für uns nicht» (405; das Subjekt im Dual wird durch das zum Wort 'Zeit' hinzugefügte Possessivsuffix ausgedrückt). ${ }^{1}$

Als kurze Zusammenfassung will ich betonen, dass der Ausdruck "Konjugation" auf das Juraksamojedische bezogen nicht dasselbe bedeutet, was er in den indogermanischen und in den finnisch-ugrischen Sprachen bedeutet. Für das Juraksamojedische ist die Konjugation nichts anderes als eine Systematisierung der Erscheinungsformen der Verbalnomina mit prädikativer Funktion. In ihrer prädikativen Funktion haben die Verbalnomina nominalen Charakter, ihre Bedeutung ist die eines nomen actionis bzw. nomen acti. (Die Bedeutung eines nomen agentis lässt sich nicht einmal im Indikativ bestätigen.)

Die Konjugationen der intransitiven und der transitiven Verba unterscheiden sich im wesentlichen nicht voneinander. In den Formen der 3. Sg. erscheint teils der Verbalnomenstamm: 'Gehen'; 'Geben' (= "subj. Konj."), teils das mit dem Possessivsuffix versehene Verbalnomen: 'sein Gehen' (= "refl. Konj.»); 'sein Geben' (= "obj. Konj.»). Die Wirklichkeit (= Indikativ) wird durch das mit dem Suffix $* j \varepsilon(i)$ gebildete Verbalnomen aktiver Bedeutung ausgedrückt. Im Konjunktiv bezeichnet das Suffix ${ }^{*} j \varepsilon(i)$ teils den nominalen Charakter des Verbalnomens, teils die Passivität. Verbalnomina, deren Suffixe ein ${ }^{*} \beta$-Element enthalten, drücken einen $\mathbf{Z u}$ stand passiver Bedeutung aus. Den Zustand bezeichnen Verbalnomina, deren Suffixe ein ${ }^{*} m$.- bzw. ein ${ }^{*} p$.-Element haben. Verbalnomina auf ${ }^{*} n a,{ }^{*} n \varepsilon$ haben die Bedeutung des Potentialis, Konditionalis und Optativs. Den Konditionalis drücken Verbalnomina aus, deren Suffixe ein *'s.-Element enthalten. Ein Desiderativum bezeichnen Verbalnomina, deren Suffixe ein $* k$.-Element haben, sowie Verbalnomina auf

1 Äusserst selten begegnet eine eigenartige lativische Form: das Suffix des Konditionalis erscheint nach dem Possessivsuffix der 2. Pl.: (C) "tjetam njinjikāmdōh | häda-uan-da-dsi, | uäskom nisjamda’ah | häda$u$-wan-da-dsi weure vier älteren Brüder | sollt ihr erschlagen haben, | euren alten Vater | sollt ihr erschlagen haben" (283). 
*ta. Alle diese Suffixe zusammen mit den Suffixen *l. und *r. (die in sekundären Verbalstämmen erscheinen,) sind uralischer Herkunft und vertreten die älteste Schicht der Suffixe. Die Verbalnomina in prädikativer Funktion sind alle achronistisch mit der Einschränkung, dass die eventuelle Erfüllung des Desideriums vom Standpunkt des Sprechers nur in der Zukunft erfolgen kann. -. Die deskriptiven Grammatiken helfen - wie es N. M. Tereščenko sehr treffend formuliert hat - - mit den Kategorien des Tempus denjenigen, die sich den achronistischen Zustand nicht vorstellen können.

IRENE N.-SEBESTYÉN 